\title{
Diffractive Optics for thin-film silicon solar cells
}

Christian Stefano Schuster

Doctor of Philosophy

University of York

Physics

May 2015 


\section{Abstract}

Thin-film silicon solar cells have the potential to convert sunlight into electricity at high efficiency, low cost and without generating pollutants. However, they need to become more competitive with conventional energy technologies by increasing their efficiency.

One of the key efficiency limitations of using thin silicon absorber materials relates to the optical loss of low-energy photons, because the absorption coefficient of silicon decreases strongly for these low-energy photons in the red and nearinfrared, such that the absorption length becomes longer than the absorber layer thickness. If, in contrast, the incident light was redirected and trapped into the plane of the silicon slab, a thin-film could absorb as much light as a thick layer.

Diffractive textures can not only efficiently scatter the low-energy photons, but are also able to suppress the reflection of the incident sunlight. In order to take advantage of the full benefits that textures can offer, I outline a simple layer transfer technique that allows the structuring of a thin-film independently from both sides, and use absorption measurements to show that structuring on both sides is favourable compared to structuring on one side only.

I also introduce a figure-of-merit that can objectively and quantitatively assess the benefit of the structuring itself, which allows me to benchmark state-ofthe-art proposals and to deduce some important design rules. Minimising the parasitic losses, for example, is of critical importance, as the desired scattering properties are directly proportional to these losses. To study the impact of parasitics, I quantify the useful absorption enhancement of two different light trapping mechanisms, i.e. diffractive vs plasmonic, based on a fair and simple experimental comparison. The experiment demonstrates that diffractive light-trapping is a better choice for photovoltaic applications, because plasmonic structures accumulate the parasitical losses by multiple interactions with the trapped light.

The results of this thesis therefore highlight the importance of diffractive structures as an effective way of trapping more light in a thinner solar cell device, and will help to define guidelines for new designs that may overcome the $30 \%$ power conversion efficiency limit. 


\section{Contents}

Abstract

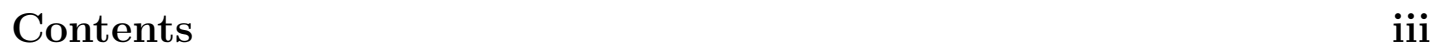

List of Figures viii

List of Tables $\quad$ xi

$\begin{array}{ll}\text { Acknowledgements } & \text { xiii }\end{array}$

Declaration of Authorship xvi

1 Introduction $\quad 1$

1.1 Introduction . . . . . . . . . . . . . . . . 1

1.2 The potential of photovoltaics . . . . . . . . . . 1

1.3 Light trapping for thin film silicon solar cells . . . . . . . . . . . 4

1.3.1 The NIR problem . . . . . . . . . . . . . . . 4

1.3.2 The light-trapping potential . . . . . . . . . . . . 7

1.4 Scope \& outline of this thesis . . . . . . . . . . . . . . 12 
2 Nanostructures for enhanced light-trapping in thin-film silicon solar cells

2.1 Introduction . . . . . . . . . . . . . . . . . . 13

2.2 The main light-trapping approaches . . . . . . . . . . . . . 14

2.2.1 Non-diffractive techniques . . . . . . . . . . . . 14

2.2.2 Refractive approaches . . . . . . . . . . . . 17

2.2.3 Diffractive approaches . . . . . . . . . . . . . . . . 19

2.2.3.1 The grating equation . . . . . . . . . . 20

2.2.3.2 Discussion . . . . . . . . . . . . . 21

2.3 The Lambertian Scatterer . . . . . . . . . . . . . . . . . 23

2.3.1 Absorption enhancement by a Lambertian backscatterer . 26

2.3.1.1 The Lambertian Limit . . . . . . . . . . . . . . 29

2.3.2 The maximum absorption enhancement . . . . . . . . . . 32

2.3.2.1 Over the limits . . . . . . . . . . . . 34

2.4 Assessment of light-trapping . . . . . . . . . . . . . . 35

2.4.1 How to assess light-trapping structures for solar cells ? . . 35

2.4.2 The light-trapping-efficiency (LTE) . . . . . . . . . . . . 39

2.4.2.1 The effective thickness . . . . . . . . . . . 40

2.5 State of the art . . . . . . . . . . . . . . . 42

2.5.1 Important milestones . . . . . . . . . . . . . . . . . 42

2.5.2 The state of the art . . . . . . . . . . . . . 49

2.6 Concluding remarks . . . . . . . . . . . . . . . . . . . 54

2.6.1 Phase engineering . . . . . . . . . . . . . . 54

2.6.2 Dual structuring . . . . . . . . . . . . . . 56

2.6.3 Electrical performance . . . . . . . . . . . 56 
3 Fabrication and characterisation of diffractive nanostructures $\quad 58$

3.1 Introduction . . . . . . . . . . . . . . . . . . 58

3.2 Nanolithography techniques . . . . . . . . . . . . . 58

3.2.1 Electron beam lithography . . . . . . . . . . . . 59

3.2.1.1 The fabrication of diffractive structures . . . . . 60

3.2 .2 Nanoimprint lithography . . . . . . . . . . . . . 61

3.2.2.1 The imprint step . . . . . . . . . . . . 62

3.2.2.2 The demoulding step . . . . . . . . . . . 64

3.3 Pattern transfer by reactive ion etching (RIE) . . . . . . . 66

3.3.1 Dry-etching in the RIE-system . . . . . . . . . . . . . 67

3.4 Absorption measurements . . . . . . . . . . . . . . . 69

3.4.1 Reference measurement - thin films . . . . . . . . . 70

3.5 Summary . . . . . . . . . . . . . . . . . . 72

$\begin{array}{llr}4 & \text { Achievements } & 73\end{array}$

4.1 Introduction . . . . . . . . . . . . . . . 73

4.2 Dual gratings by a simple layer transfer technique . . . . . . . . . 74

4.2.1 Why dual gratings ? . . . . . . . . . . . . 75

4.2.2 A novel layer-transfer technique . . . . . . . . . . 75

4.2.3 Proof of principle demonstration . . . . . . . . . . 77

4.2.3.1 Fabrication and design parameters of the dualgrating ................. 77

4.2.3.2 Measurements and results . . . . . . . . . 80

4.2.3.3 Conclusions . . . . . . . . . . . . . . . . . 82

4.3 Plasmonic and diffractive nanostructures for light trapping - an experimental comparison . . . . . . . . . . . . . 82 
4.3 .1 Methodology ................. 83

4.3.1.1 The diffractive design . . . . . . . . . . . . 84

4.3.1.2 The plasmonic design . . . . . . . . . 85

4.3.2 Results........................ . . 85

4.3.2.1 Material properties ............ 85

4.3.2.2 Absorption measurements . . . . . . . . . 87

4.3 .3 Discussion . . . . . . . . . . . . . . . . 88

4.3.3.1 Extraction of the parasitic influences . . . . . . 90

4.3.4 Comparison of the two different approaches . . . . . . . . 92

4.3.5 Conclusions . . . . . . . . . . . . . . . . . . . . . 94

4.4 Summary . . . . . . . . . . . . . . . . . . 96

$\begin{array}{lll}5 & \text { Conclusions and outlook } & 98\end{array}$

5.1 General conclusions and remarks . . . . . . . . . . . 98

5.1.1 Limitations and future work left . . . . . . . . . . 101

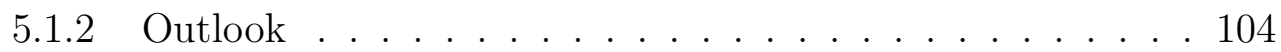

5.2 Can solar power solve the world's energy crisis ? . . . . . . . 106

Appendices

A A home-made UV nanoimprint tool . . . . . . . . . . . . . 109

A.1 Technical requirements . . . . . . . . . . . . . 109

A.2 Technical specifications of the UV tool . . . . . . . . . 111

A.3 Testing the tool by photo lithography . . . . . . . . . . 112

A.4 The stamp materials . . . . . . . . . . . . . 112

A.5 Testing the tool by nanoimprint lithography . . . . . . . . 115

A.6 Concluding remarks . . . . . . . . . . . . . . . 117 
B Absorption measurement setup . . . . . . . . . . 118

B.1 Design considerations . . . . . . . . . . . . . 118

B.1.1 The integrating sphere . . . . . . . . 118

B.1.2 The monochromator . . . . . . . . 120

B.1.3 The detectors . . . . . . . . . . . . 123

Detector bandwidth . . . . . . . . . . . 123

Sensitivity . . . . . . . . . . . . . . 124

B.2 Critical assessment . . . . . . . . . . . . . 127

B.3 Data acquisition - speed and accuracy . . . . . . . . . 130

B.4 Measurement limitations . . . . . . . . . . . . . 130 


\section{List of Figures}

1.1 The price-experience curve for silicon solar modules . . . . . . . . 3

1.2 The increase of transmission losses by a thinner absorber layer . . 5

1.3 The indirect nature of the silicon bandgap . . . . . . . . . . . 6

1.4 The benefits of light-trapping for solar cells . . . . . . . . . . . . . 11

2.1 Silicon pillar solar cell arrays . . . . . . . . . . . . . . . . . . 14

2.2 Nanowire, Mie resonator and plasmonic light-trapping techniques 16

2.3 Anti-reflective textures . . . . . . . . . . . . . . 18

2.4 Refractive textures with anti-reflective moth-eye effect $\ldots . . .19$

2.5 Diffraction at a narrow opening . . . . . . . . . . . 20

2.6 Diffraction of light by a periodic surface-reflief grating . . . . . . . 22

2.7 Definition of a Lambertian Scatterer . . . . . . . . . . . . . 24

2.8 The Lambertian model . . . . . . . . . . . . . . . . . . . . . 25

2.9 The Lambertionality factor . . . . . . . . . . . . . . . . . 29

2.10 The average scattering angle of a Lambertian texture . . . . . . . 33

2.11 The Lambertian Limit as a function of the absorber thickness . . 38

2.12 The thickness definition for the $L T E \ldots \ldots$. . . . . . . . . . . 42

2.13 The first $20 \%$ efficient silicon solar cell . . . . . . . . . . . . . 44

2.14 High efficiency multicrystalline Si solar cell by texturing . . . . . 47 
2.15 Publication records of light-trapping for solar energy . . . . . . . 48

2.16 Random surface textures . . . . . . . . . . . . . . . . . 49

2.17 The $L T E$ of structures realized or proposed in c-Si . . . . . . . 51

3.1 Electron beam lithography . . . . . . . . . . . . . . . 60

3.2 Nanoimprint lithography . . . . . . . . . . . . . . 63

3.3 Comparison of different etching techniques . . . . . . . . . 67

3.4 Reference measurement $-1 \mu \mathrm{m} \mathrm{c-Si} \ldots \ldots 71$

3.5 Reference measurement $-220 \mathrm{~nm} \mathrm{c-Si} \mathrm{\ldots .} \mathrm{.} \mathrm{.} \mathrm{.} \mathrm{.} \mathrm{.} \mathrm{.} \mathrm{.} \mathrm{.} \mathrm{.} 71$

4.1 A novel, simple and effective layer transfer technique . . . . . . 78

4.2 Top and bottom photonic lattice of the dual-grating approach . . 79

4.3 Fabricated dual-grating structure . . . . . . . . . . . 79

4.4 Absorption of unstructured a-Si:H . . . . . . . . . . . . . 80

4.5 Absorption of dual-structured a-Si:H . . . . . . . . . . . 81

4.6 Plasmonic scattering vs diffractive optics . . . . . . . . . . 84

4.7 Optical material function $n$ and $k$ of a-Si . . . . . . . 86

4.8 Raman spectra of a-Si . . . . . . . . . . . . 86

4.9 Measured total absorption of the structured a-Si samples . . . . . 87

4.10 Extended lossy waveguide model (parasitic absorption included) . 89

4.11 Modelling the parasitic absorption caused by Ag nanoparticles . . 91

4.12 Assessment with wavelength dependent fitting parameters . . . . 92

4.13 Assessment of the useful absorption . . . . . . . . . . . . . 93

5.1 Wet-etching of thick Si wafers . . . . . . . . . . . 103

5.2 Fabrication process for thin-film silicon solar cells . . . . . . . 105

5.3 Energy requirements in light of the global ecological footprint . . 108 
A.1 Basic requirements of a UV nanoimprint tool . . . . . . . . . 109

A.2 Home-made nanoimprint setup . . . . . . . . . . . . . . 110

A.3 The nanoimprint process with AMONIL resist and PDMS stamp . 115

A.4 The UV nanoimprint lithography test with PDMS and AMONIL . 116

B.1 Integrating sphere setup . . . . . . . . . . . . . . . 119

B.2 The USB4000 sensor from Ocean Optics . . . . . . . . . . . 121

B.3 The white light laser source . . . . . . . . . . . . . . . . 122

B.4 The diffraction grating of the monochromator . . . . . . . . . 122

B.5 Arrangement of the detectors for the absorption measurement . . 123

B.6 The transimpedance amplifier . . . . . . . . . . . . 125

B.7 Absorption measurement setup . . . . . . . . . . . . . . . 129 


\section{List of Tables}

2.1 The $L T E$ of numerical structures using c-Si . . . . . . . . . . 53

2.2 The $L T E$ of experimental structures using c-Si . . . . . . . . . . . 54

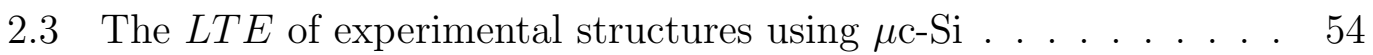

3.1 The three major nanoimprint lithography techniques . . . . . . 64

3.2 RIE system at York . . . . . . . . . . . . . . . . . . . 68

4.1 Measured absorption enhancement of a structured $400 \mathrm{~nm}$ thin aSi:H slab . . . . . . . . . . . . . . . . . . . . . 81

4.2 LTE of the plasmonic and diffractive structure . . . . . . . . . 94

A.1 Specifications of the LED array used in the UV tool . . . . . . . 111

A.2 Key requisites for UV nanoresists . . . . . . . . . . . 117

B.1 The Si photodetectors . . . . . . . . . . . . . 128

B.2 Assessment of the detectors' key parameters . . . . . . . . . . 129

B.3 Optimal parameter settings for the measurement of $A(\lambda) \ldots 130$ 
"It's never as good as it seems, and it's never better."

George Bernard Shaw 


\section{Acknowledgements}

Since no one has ever achieved anything worthwhile without help, no duty is more urgent than that of returning thanks. I would like to thank with the following lines all the people who made this thesis possible and supported my studies.

First and foremost I want to thank my supervisor Prof. Thomas Krauss. He guided me with his wide experience and deep knowledge in photonics through many challenges of the thesis. I appreciate all the time you took for discussions (even for long ones), admire your inspiration, sage advice, steady motivation to solve even the most difficult research puzzle and I am extremely thankful for your outstanding continuous help in innumerable ways. It was a privilege and honour to perform my $\mathrm{PhD}$ under your supervision; one simply could not wish for a better supervisor !

A successful end of this $\mathrm{PhD}$ project, however, would never ever have been possible without the support, encouragement and assistance from a lot of people. I would regret not to have listed each and every one of them, because you all helped me to progress in my research.

For example, I would not have even started this $\mathrm{PhD}$ without the existence of the Marie-Curie PROPHET project. My sincere gratitude goes to David Williams for providing and managing this enjoyable EU project. It was a unique, amazing experience to collaborate and to work in a training network with so excellent research partners. Of course, I will never forget our ascent of mount Etna with Guillaume Huyet in Sicily!

I am indebted to a few invaluable team members who have supported me to tackle scientific problems in creative ways: Marcello Ferrera, Andrea Di Falco, William Whelan-Curtin (Liam O'Faolain), Emiliano Rezende-Martins and Christopher Reardon. I have enjoyed the opportunity to watch and learn from your knowledge and experience.

It has also been a privilege to work closely with Daan Stellinga and Graham Triggs; the atmosphere of our office was always motivating and never boring and I like to add big thanks to Annett Klemm and Bryan O'Regan - when will be bag the next munro? 
I further owe Peter Reader-Harris and Christian Reimer a debt of gratitude for the many lively and philosophical discussions at the harbour of St Andrews during unforgettable lunch breaks that truly refilled our research engines. I also like to take this opportunity to acknowledge the ideas from Kezheng Li, Alberto Jimenez and Mark Scullion, who turned my Yorkshire breaks into inspiring brainstorms. Many thanks to all of you !

Actually, most of the results described in this thesis would not have been obtained without a close collaboration with few laboratories.

I am greatly thankful of the wise counsel of Prof. Lucio Andreani and Dr. (!) Angello Bozzola from the University of Pavia (Italy), and I am very proud of what we have achieved together. It was also a great learning curve for me to study important modelling and simulation aspects from Marco Liscidini and Piotr Kowalczewski - thank you so much! My sincere thanks to Maddalena Patrini and Scott Robertson for their friendly and immeasurable support.

The joint research efforts with the CNR-IMM Catania (Italy) were only a success, because of Isodiana Crupi, Manuel Mendes and Seweryn Morawiec. I have learned a lot about plasmonics from you. Thank you for all your patience, but also for preparing me the best Paella dinner of my life.

One of the most interesting and instructive interactions I had was with the members of the research team of 3SUN in Catania (Italy), who became a second family to me. It is my greatest pleasure to thank Cosimo Gerardi, Andrea Canino, Dario Rapisarda, Giuseppe Condorelli and Marina Foti for giving me useful and superb insights of "real photovoltaics" and I am even lost for words to express my gratitude to Anna Battaglia. All the good moments we have shared (and that were many) will be always with me.

Andrea Cattoni from the CNRS/LPN Marcoussis (France) deserves a sincere and huge thank you, because you have given me invaluable support in setting up my nanoimprint process when good advice was hard to find. Many thanks go to Andy West, who helped me in characterizing the spectrum of the nanoimprint tool in the York Plasma Institute Laboratories (YPIL). I also wish to thank Marco Vogler from Microresist (Germany), who kindly provided me with a free sample of nanoresist. I extend my thanks to Ounsi ElDaif, who made one of my conference visits to a memorable success. 
However, I could not have done this thesis without the many brilliant technicians. In fact, the technical support was far beyond duty many times. I send my deep thanks to Steven Balfour, Callum Smith, George Robb, Dave Coulthard, Mark Laughton, Bob Hide and Neil Johnson. I am so thankful that you shared your skills and time with me - not only at work. However, although your contributions to this dissertation are very vital, I keep the mistakes in it for my own. Honestly, I do not know how I could have finished my $\mathrm{PhD}$ without you.

Most of all, a very special thank to all my old and new friends outside of the university world for their never ending support. Harrold, how can I now start a day without you and the best guide dog on Earth? Huge thanks to Gordon and Greg for all the awesome weekends together. You were the best neighbours I have ever had - I am missing you soooo much! However, how could I have survived without my house mates Susan and John? Many thanks to Sierra, Gaynor and Dougie, who made my life looking bright in Fife. Marco and Kerim, thank you for sharing with me the best glide sides in Sicily and UK. Thank you Vivian (Zhan Wei Scullion) for your support and your friendship, thank you Stefan for introducing me the best board games on the planet, thank you Martin for your enormous friendship and for everything you did for me... you are all making even the dullest day seem merrier.

Last but not least, my final and greatest thanks go to my dear beloved parents, Laura and Werner, and my brother Marco for their constant love, never ending support and care throughout my many years of studies (far away from home) and through the process of researching and writing this PhD thesis. You encouraged me to do what makes me happy and to follow where my heart takes me. And finally, Sara, you are my foundation, please hold my hand and heart forever. 


\section{Declaration of Authorship}

I hereby declare that this thesis titled, 'Diffractive optics for thin-film silicon solar cells' and the work presented in it are my own. I confirm that:

- This work was done wholly or mainly while in candidature for a research degree at this University.

- No part of this thesis is concurrently submitted in candidature for any degree other than Doctor of Philosophy of the University of York.

- Where I have consulted the published work of others, this is always clearly attributed.

- Where I have quoted from the work of others, the source is always given. With the exception of such quotations, this thesis is entirely my own work.

- I have acknowledged all main sources of help.

- Where the thesis is based on work done by myself jointly with others, I have made clear exactly what was done by others and what I have contributed myself. 
Dedicated to the photon that entangled me with the love of my wife. 


\section{Chapter 1}

\section{Introduction}

\subsection{Introduction}

This chapter tries to answer the question why research into silicon photovoltaics is important in section 1.2, to quantify the potential of light trapping in section 1.3 and will outline the content and scope of my thesis in section 1.4.

\subsection{The potential of photovoltaics}

Photovoltaics is able to convert sunlight directly into electricity and so allows to create an independent power supply. Solar cells can generate power in remote areas, because they do not rely on energy sources other than sunlight. In principle, the average sunshine over an area only as large as Wales (UK) would already be sufficient to supply the world's electricity needs.* Photovoltaics thus has a great potential to address the world's energy requirement in the long run, in contrast to conventional resources, which are either finite or which generate pollutants. However, in order to compete with conventional energy technologies, solar cells still need to prove higher power conversion efficiencies and lower costs.

\footnotetext{
* own calculations, based on $2.4 \mathrm{kWh} / \mathrm{m}^{2}$ on an average day from sun
} 
If I first consider what limits the conversion efficiency of sunlight into electricity, optical losses are identified as one of the main loss mechanisms, which will be the focus of this thesis. Thermal loss mechanisms, such as Joule heating, are another key factor affecting the power conversion efficiency. The need for good electrical transport properties does not tolerate material impurities or fabrication imperfections, hence electrical loss mechanisms are also important, especially as the cost-pressure in the solar industry makes it often too expensive for industry to use high purity materials.

While my work does not explicitly address the electrical loss mechanisms, I note that the optical light trapping techniques studied in this thesis allow us to use less material, thereby reducing the impact of material defects, as thinner films make it easier for charge carriers to be collected. I also note that a more efficient usage of silicon is not the cost driver any more, because the raw material now is only $20 \%$ of the module cost and less than $10 \%$ of the total cost of a photovoltaic system [1].

As performance increases and manufacturing costs decline over time, the ratio of cost to performance, expressed in US Dollars per peak Watt generated by a module $\left(\mathrm{US} \$ / \mathrm{W}_{\mathrm{p}}\right)$, is taken as a standardized figure-of-merit to quantify the improvements in photovoltaics. Figure 1.1 uses this definition to illustrate how photovoltaics has continued to follow a trend, that makes the technology ever more competitive, especially as the cost of fossil fuels tends to increase (despite the drop in oil price in 2015), leading now to grid parity in the southern part of Europe [2]. Grid parity means that the cost of solar electricity is equal to the cost of electricity from the national grid transmission network.

If research and development in photovoltaics aims to follow this trend curve, neither high efficiency nor cost reduction is sufficient to displace the current technology. I cite two examples to illustrate this point.

1. High efficiency. Using a $1 \mu \mathrm{m}$ thick GaAs slab, the US based company Alta Devices set a world record efficiency of $29 \%$. Nevertheless, high efficiency is not enough, as the GaAs slab is more expensive to produce than comparable silicon cells (in terms of US\$/ $\mathrm{W}_{\mathrm{p}}$ ). Hence, these world record cells are now not used in solar farms, but in consumer electronics instead, where surface area is premium. Alta Devices was recently sold to the Chinese company Hanergy, who intend to use this technology as a solar gadget [3]. 
2. Cost reduction. Nanosolar, Abound Solar and Solyndra tried to commercialize low-cost solar modules. The companies were able to increase the throughput of their manufacturing plants by improving the speed of their deposition process, producing high quality CIGS and CdTe material very efficiently. When the modules were ready for the market, the prices for crystalline silicon modules had dropped in the meantime, and the loss of cost advantage ultimately forced the companies to shutdown [4].

A new development in photovoltaics therefore needs to move below the trend curve of silicon wafers; in order to be competitive and to justify investments.

One way to achieve this is to reduce the material thickness, thereby reducing material cost, while maintaining cell efficiency. This is the strategy of the US company Solexel [5], who are aiming to introduce a $22 \%$ efficient silicon solar cell with only $35 \mu \mathrm{m}$ silicon thickness, aiming for $0.42 \mathrm{US} \$ / \mathrm{W}_{\mathrm{p}}$. This means that their cells would be as efficient or better than State-of-the-Art bulk silicon cells, while costs are lower. Their cell rely on light-trapping, i.e. on increasing the absorption length by efficiently scattering the light inside the cell.

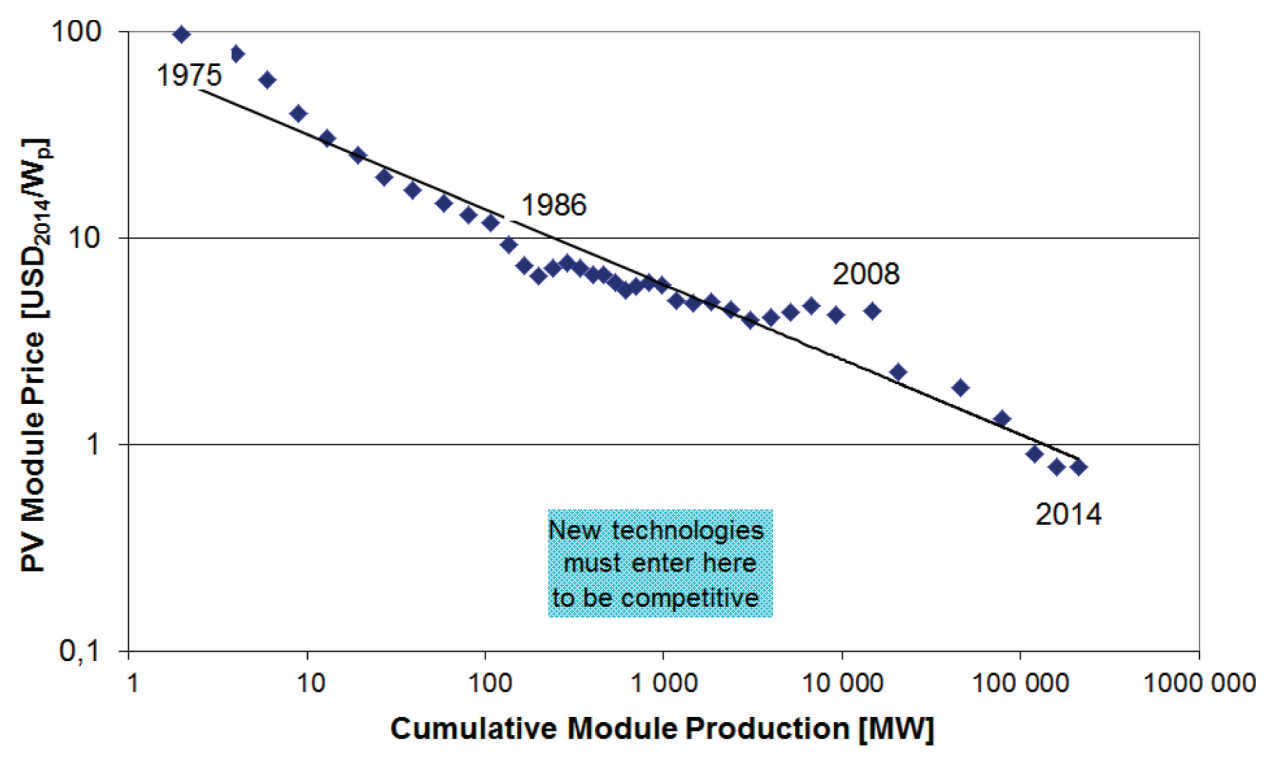

FIG. 1.1: The ratio of cost to generated power in $\mathrm{US} \$ / \mathrm{W}_{\mathrm{p}}$ is used to quantify the improvement in photovoltaics. The graph illustrates the case of silicon wafer technology, which represents ca. $90 \%$ of the global market share. The trend curve is indicated by a black line and highlights the rapid growth rate of the cumulative capacity worldwide: for each doubling of production volume, the average selling price of solar modules has fallen by $20 \%$ [2]. 


\subsection{Light trapping for thin film silicon solar cells}

Light trapping aims to overcome the problem of low absorption of red and nearinfrared photons (the NIR problem) in silicon. This low absorption is a particular problem for thin silicon absorbers. I will first analyse the problem in terms of the optical depth that defines the onset of photon absorption, i.e. the optical bandgap of a material. Light-trapping can then be understood as a means of lowering the optical bandgap of silicon. This interpretation will allow me to provide a more general discussion about the potential benefit of light-trapping in terms of higher power conversion efficiencies.

\subsubsection{The NIR problem}

The near-infrared (NIR) problem stands for the loss of opacity by a thinner silicon slab. If the amount of material is reduced, low-energy photons are less likely to be absorbed, because they require a longer absorption length in a silicon slab compared to more energetic photons.

In order to distinguish the threshold for photon absorption from the electronic bandgap $E_{g}$, authors often quote the optical bandgap $E_{o}$ instead. Although both quantities are understood as independent of the absorber thickness, thinfilm silicon solar cells suffer from optical losses in the NIR, because the onset $E_{o}$ essentially depends on the layer thickness, as illustrated in Fig. 1.2 for silicon.

The thickness dependence of $E_{o}$ is caused by the indirect nature of the electronic bandgap. In an indirect bandgap material such as silicon, a transition always involves both photons and phonons, as schematically shown in Fig. 1.3.

If an electron absorbs a photon, it first occupies a metastable state within the bandgap from where it will then scatter into the conduction band via phonon interaction. The wavelength dependent absorption coefficient $\alpha(\lambda)$ could thus be derived from the probability of all relevant inter-particle interactions [6]. In consequence, transitions between the valence and conduction band are less likely compared to a direct bandgap material like GaAs, because direct transitions do not require phonon interactions. 


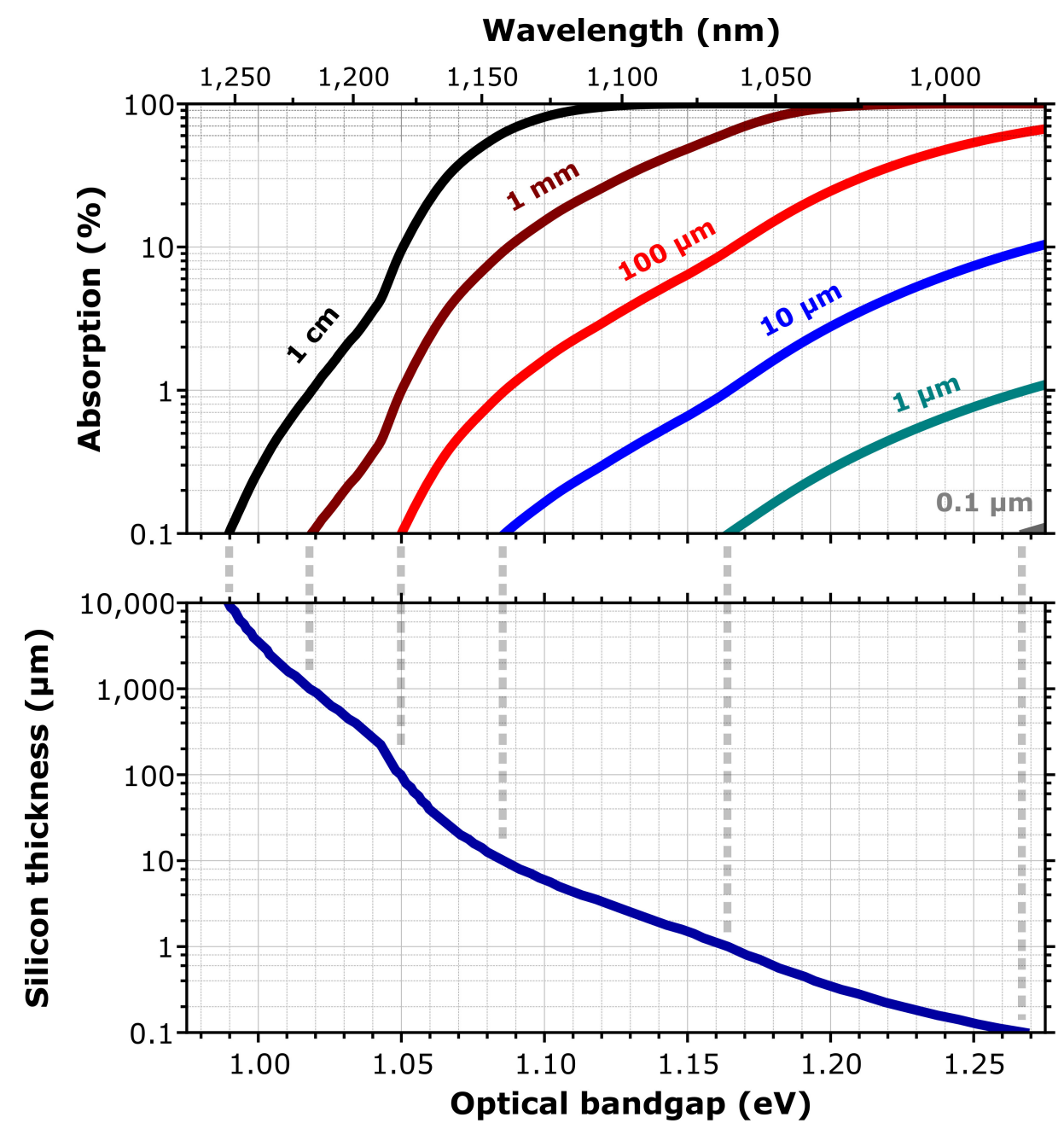

Fig. 1.2: The optical bandgap $E_{o}$ is defined as the threshold for photon absorption $(A>0.1 \%)$, i.e. when the optical depth $\tau_{\mathrm{dB}}=-10 \cdot \log (1-A)$ exceeds $0.01 \mathrm{~dB}$, which is cited as the theoretical loss factor of modern optical fibre communication [7]. The optical depth is the product of the absorber thickness and the wavelength dependent absorption coefficient. The thinner the silicon absorber layer, the lower the optical depth and the higher the optical bandgap, consequently, the more low-energy photons will pass through the medium without being absorbed. 


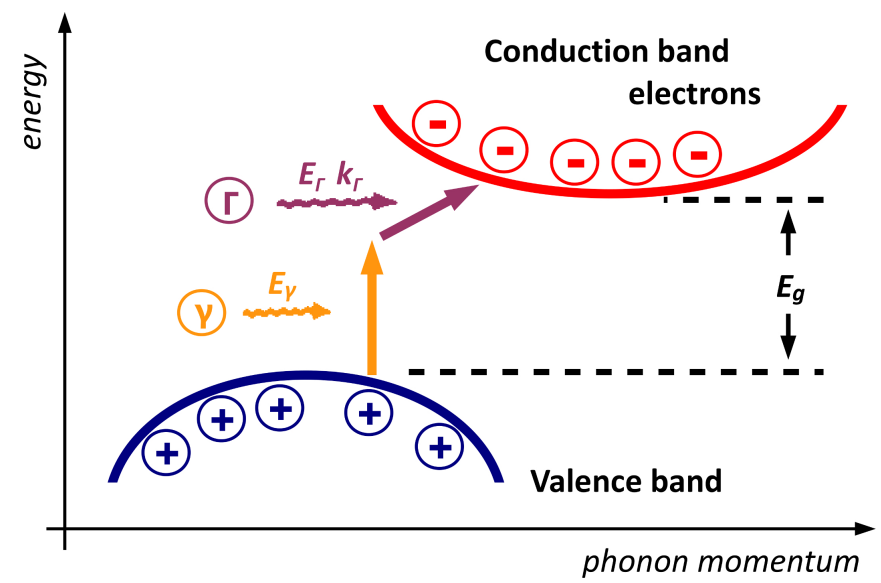

FIG. 1.3: In a semiconductor with indirect bandgap $E_{g}$, like silicon, the charge carriers in the valence and conduction band differ by a (reciprocal) lattice vector $k$. Since momentum has to be conserved in band-to-band transitions, the absorption of a photon with energy $E_{\gamma}$ requires the supply of a lattice vibration (phonon) with momentum $k_{\Gamma}$ and energy $E_{\Gamma}$. The optical threshold is then given by $E_{\gamma}=E_{g}-E_{\Gamma}$. The phonon energy is usually small, and tends to get neglected at room temperature $\left(E_{\Gamma} \ll E_{g}\right)$.

Since the probability of a transition scales as the slab thickness $d$, the product $\alpha d$ is called the optical depth of a medium

$$
\tau(\lambda) \equiv \int_{0}^{d} \alpha(\lambda, x) \cdot \mathrm{d} x
$$

and is expressed in units of decibels. The optical depth quantifies the opacity of a material for a particular wavelength. For example, a medium is called transparent when its optical depth is $0 \mathrm{~dB}$ for radiation passing through it. Reducing the absorber thickness while maintaining a high optical depth is therefore a key goal of light-trapping.

The optical depth, for a given material thickness, can be increased by increasing the path integral in Eq. 1.1. For example, the addition of light trapping increases the path inside the absorber material, thereby increasing the path integral and the optical depth. Since a larger optical depth translates into a higher absorption, especially of low energy photons, light-trapping can be understood as a means of lowering the optical bandgap $E_{o}$. 
It is then a valid question, whether and to what extend the potential benefit of a lower optical bandgap translates into a higher power conversion efficiency of a silicon solar cell. The impact of light-trapping on the power conversion efficiency is therefore analysed next.

\subsubsection{The light-trapping potential}

Solar cells convert the absorbed solar energy into an electric charge with the potential to drive a load. The maximum potential energy that can be delivered to a load is estimated by the energy $e V_{o c}$ when the load remains unconnected in the electric circuit, i.e. at open circuit conditions. If a solar cell is operated at open circuit, the converted energy from sunlight can neither generate an electric current flow nor deliver a voltage across the load. Consequently, all the energy absorbed from the sun would be balanced (ideally) by an equivalent emitted energy flux back towards the sun, which is well-known as Kirchhoff's equilibrium law of radiation.

Since the rate at which photons are absorbed is then equal to their rate of emission, the photons in the absorber are in a thermodynamic equilibrium with the excited charge carriers. In this equilibrium, the probability of creating an electron-hole pair $e+h$ (by the absorption of a photon) must be equal to the probability of photon emission $\gamma$ (by the recombination of an electron-hole pair):

$$
\gamma \rightleftharpoons e+h
$$

Since the potential for a particle to undergo a reaction is quantified by its chemical potential, the chemical potential of the emitted photons $\mu_{\gamma}$ in the absorber must be equal to the chemical potential of the electron-hole pairs, which is the maximum potential energy that can be delivered to a load $e V_{o c}$,

$$
\mu_{\gamma}=e V_{o c} \quad \text { in photon equilibrium, }
$$

assuming no heat loss occurs. P. Würfel [8] explains this chemical equilibrium in analogy to a hypothetical hydrogen fuel cell, where water molecules are split 
by high-energy photons into oxygen and twice as many hydrogen molecules. As long as the decomposition occurs at the same rate as the reverse reaction, and neither water, hydrogen nor oxygen molecules are removed (closed system), the chemical potential of all reaction partners is non-zero under irradiation.

The average number of photons in a certain energy interval is given by the BoseEinstein distribution function, such that the flow equilibrium (Kirchhoff relationship) between absorbed and emitted photons now reads

$$
\int_{E_{o}}^{\infty} A(\varepsilon) \frac{\varepsilon^{2}}{e^{\varepsilon / k_{B} T_{S}}-1} \cdot d \varepsilon \propto \int_{E_{o}}^{\infty} A(\varepsilon) \frac{\varepsilon^{2}}{e^{\left(\varepsilon-e V_{o c}\right) / k_{B} T}-1} \cdot d \varepsilon .
$$

Here, $A$ stands for the silicon absorption, $\varepsilon$ for the photon energy, $E_{o}$ is the optical bandgap or the threshold for photon absorption, $k_{B}$ the Boltzmann constant, $T$ the temperature of the absorber and $T_{S}$ the temperature of the sun. For silicon, it is $E_{o}-e V_{o c} \gg k_{B} T$ and Eq. 1.2 simplifies to

$$
\int_{E_{o}}^{\infty} A(\varepsilon) \frac{\varepsilon^{2}}{e^{\varepsilon / k_{B} T_{S}}-1} \cdot d \varepsilon \propto e^{e V_{o c} / k_{B} T} \cdot \int_{E_{o}}^{\infty} A(\varepsilon) \varepsilon^{2} e^{-\varepsilon / k_{B} T} \cdot d \varepsilon .
$$

If at open circuit, the illuminated absorber is in a non-thermal equilibrium with the surroundings, the $V_{o c}$ quantifies the offset from the thermal-equilibrium situation: the larger the $V_{o c}$, the greater the photon emission rate at constant absorber temperature $T$.

In order to quantify the effect of light trapping on the open-circuit voltage $V_{o c}$, the analysis of the Kirchhoff relationship is more conveniently formulated in terms of electric currents, instead of the absorbed and emitted photon fluxes (Eq. 1.3):

1. The number of absorbed photons is measured by a solar cell under short circuit conditions, i.e. when the absorbed solar energy is converted into an electric current without potential energy to drive a load $e V_{o c}=0$. In consequence, the short-circuit current $I_{s c}$ also quantifies the maximum number of emitted photons when all photogenerated charge carriers recombine at open circuit conditions (under solar illumination), i.e. the left hand side of Eq. 1.3. 
2. As in a thermal equilibrium the electron-hole pairs are coupled to the phonons, the potential of the photons $\mu_{\gamma}$ in the absorber must also be equal to the potential of the phonon system

$$
\mu_{\gamma} \simeq 0 \quad \text { in phonon equilibrium, }
$$

since the energy per phonon is negligible small. The maximum number of thermally excited electrons and holes is measured by the short-circuit current $I_{d}$ in a dark environment. Consequently, the short-circuit current $I_{d}$ also quantifies the maximum number of emitted photons when all thermally excited charge carriers recombine at open circuit conditions (in a dark environment), which is given by the right hand side of Eq. 1.3 with $e V_{o c}=0$.

From Eq. 1.3, it now follows that the knowledge of $E_{o}$ and $A$ fixes the opencircuit voltage $V_{o c}$. However, $E_{o}$ and $A$ also define the ideal short-circuit current under light conditions $I_{s c}$, which is given by the left hand side of Eq. 1.3, and under dark conditions $I_{d}$, which is given by the right hand side of Eq. 1.3 with $e V_{o c}=0$. Together, this yields:

$$
I_{s c}\left(E_{o}\right) \propto I_{d}\left(E_{o}\right) \cdot \exp \left(\frac{e V_{o c}}{k_{B} T}\right) \quad \Longrightarrow \quad V_{o c} \propto \ln \left[\frac{I_{s c}\left(E_{o}\right)}{I_{d}\left(E_{o}\right)}\right]
$$

The open-circuit voltage therefore increases with a higher photon emission rate in photon equilibrium (nominator) or with a lower photon emission rate in phonon equilibrium (denominator). The maximum potential energy that can be delivered to a load is thus a measure of the highest operating point of a solar cell away from its thermal equilibrium position - because this equilibrium is perturbed at open-circuit conditions by sunlight.

Since light-trapping aims to enhance the absorption properties $A$ of a thin-film by lowering its optical bandgap $E_{o}$, it enhances both the absorption rate of photons from the sun (a) and the emission rate of photons into the surroundings (b):

a) The absorption enhancement in the spectral region of the optical bandgap $E_{o}$ is favourable in terms of electric current, because more charge carriers will be generated under sunlight (higher $I_{s c}$ ). 
b) The absorption enhancement in the spectral region of the optical bandgap $E_{o}$, however, also increases the number of excited charge carriers under dark conditions (higher $I_{d}$ ), thus enhances the photon emission of thermally excited charge carriers and hence reduces the offset between the phonon and the photon equilibrium. Accordingly, light trapping is then unfavourable in terms of voltage. In fact, Sandhu et al. [9, 10] present a modelling approach of nanophotonic solar cells that elucidates the physics of opencircuit voltage enhancement; they indeed show, that the enhancement was related to the absorption suppression in the immediate spectral region of the bandgap.

I am now able to discuss the potential benefit of light-trapping in terms of power conversion efficiencies: while the incident optical power of the AM1.5G solar spectrum is $100 \mathrm{~mW} / \mathrm{cm}^{2}$, the converted power is proportional to the product of $I_{s c}$ and $V_{o c}$. I therefore calculated the short-circuit current $I_{s c}$ and open-circuit voltage by solving Eq. 1.3 for $V_{o c}$, using the intrinsic material function for silicon [11], the standard AM1.5G solar spectrum and an ambient spectrum at temperature $300 \mathrm{~K}$. The outcome is shown in Fig. 1.4 and allows to study the electrical performance as a function of light trapping.

In summary, I answered the question, whether and to what extend light-trapping affects the principal operation of a silicon solar cell device by considering Kirchhoff's radiation law and the thickness dependency of the optical bandgap: lighttrapping in the NIR increases the optical depth and thereby the short-circuit current of a silicon slab, while it reduces the limiting open-circuit voltage at the same time. However, as the current enhancement is larger than the reduction in voltage, light-trapping proves to be an effective solution for the NIR-problem and allows to achieve higher power conversion efficiencies. For example, the efficiency of a $6 \mu \mathrm{m}$ thin-film of silicon would improve by $25 \%$, if light trapping allows to reduce its optical bandgap by $4 \%$, i.e. from $1.19 \mathrm{eV}$ (1040 nm wavelength) to $1.14 \mathrm{eV}$ (1090 nm wavelength). 


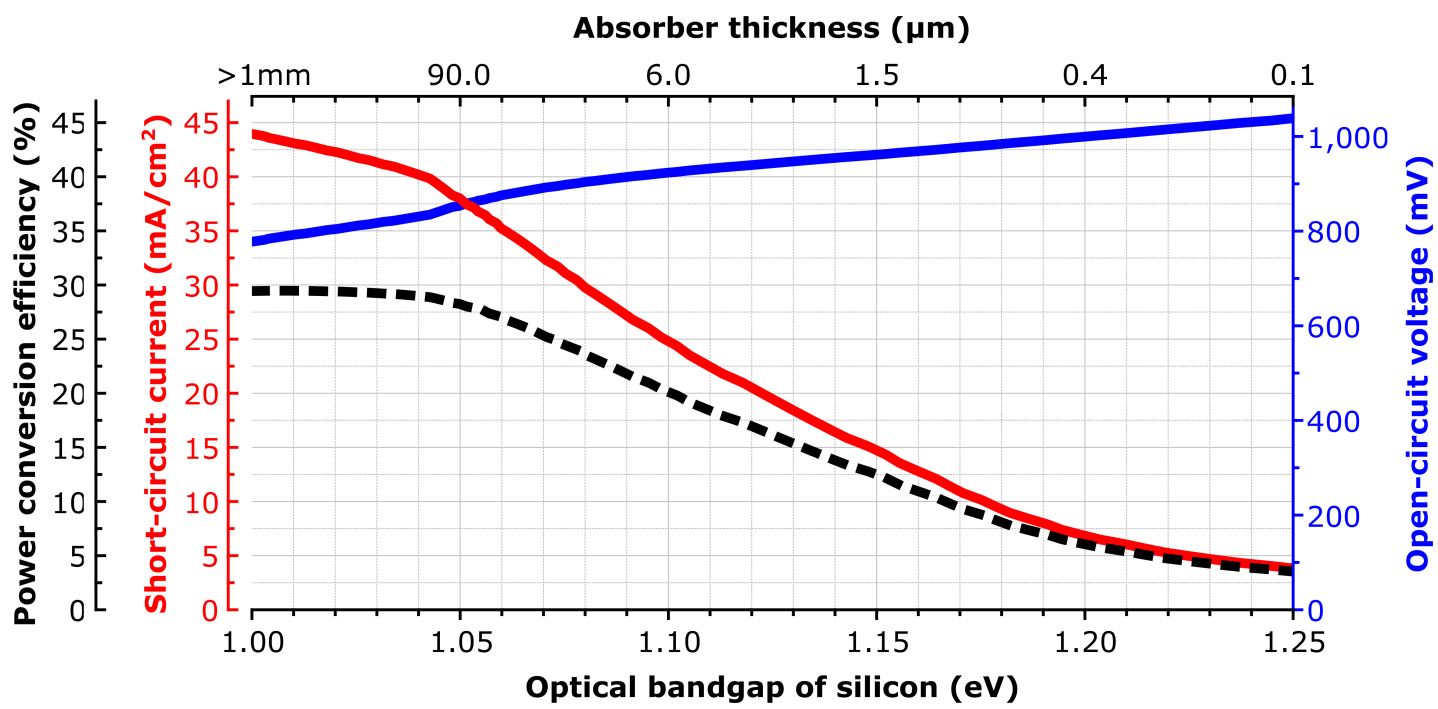

FIG. 1.4: The benefits of light-trapping for solar cells. Light-trapping is able to lower the absorption threshold, to increase the photon absorption in the NIR and, therefore, very attractive for thin-film silicon solar cells with $E_{o}>E_{g}$. Here, the optical bandgap $E_{o}$ of silicon is used as a measure for the degree of light-trapping (bottom axis); the higher the absorption, the lower $E_{o}$. For comparison, the dependence of $I_{s c}$ and $V_{o c}$ on the optical depth for a single traversal is shown as a function of the absorber thickness (top axis). While light-trapping directly translates into a higher short-circuit current $I_{s c}$ (red solid lines), it tends to reduce the open-circuit voltage $V_{o c}$ (blue solid lines) due to the enhanced emission of photons close to $E_{o}$. The black dotted line refers to the power conversion efficiency under 1 sun illumination, which is proportional to the product of $I_{s c}$ and $V_{o c}$. Light trapping proves to be an effective solution to achieve higher power conversion efficiencies, because the efficiency scales with a lower optical bandgap. All calculations are based on ideal conditions, i.e. zero surface reflection, a perfect mirror at the back side, no electrical loss mechanisms and a maximum possible fill-factor of $88 \%$ [12]. 


\subsection{Scope \& outline of this thesis}

As light trapping for thin-film solar cells has attracted a lot of research interest over the last few decades, I first present an overview over some of the main light trapping techniques in chapter 2 before focussing on diffractive light trapping approaches.

In general, light-trapping aims to increase the material absorption of incident sunlight, which naturally leads to the question of whether or not a limiting absorption enhancement factor exists. Comparing the performance of a structure against such a limiting value can then help to establish some design rules for light-trapping structures. Therefore, I will introduce and analyze the Lambertian Scatter as a useful benchmark model and a general method for the assessment of a structure, which is independent of the material, fabrication method or technology used. Applying the method to numerous proposals found in the literature then allowed me to identify the most promising light-trapping structures.

Next, in chapter 3 and section 3.4, I will introduce the methodological aspects for the fabrication and characterization of the structures used in my experiments. Although most of my diffractive structures are realized via electron beam lithography and dry etching techniques, I will also show how nanostructures can be made by nanomiprint lithography. To this end, I designed, constructed and successfully tested a home-made UV nanoimprint lithography tool.

Chapter 4 finally outlines two key experiments performed in my PhD project. The first experiment highlights a simple, effective but novel layer transfer technique. In a proof-of-principle, I demonstrated the ability to pattern a silicon thin-film independently from both sides. My technique could support the study of more efficient light trapping designs without being constraint by design parameters. The second experiment concentrates on a fair assessment of two different light-trapping mechanisms, i.e. diffractive vs plasmonic. This comparison is jointly undertaken with colleagues from the University of Catania and it highlights the importance of avoiding any optical loss mechanisms in novel lighttrapping designs.

The global conclusions of my work, some critical remarks and possible future developments are then presented in chapter 5 . 


\section{Chapter 2}

\section{Nanostructures for enhanced light-trapping in thin-film silicon solar cells}

\subsection{Introduction}

The demand for low-cost, high-efficiency solar cells along with the never-ending promises of modern technology have caused an increase of research into photovoltaics, particularly into the control of light at the subwavelength scale. In fact, the now well known Shockley \& Queisser paper about the limiting efficiency of solar cells [13] was a "sleeping beauty" for almost 40 years of slow reception [14]. Accordingly, a multiplicity of light-trapping concepts has been proposed with the intent of enhancing the optical depth of thin-film absorbers; the more lighttrapping approaches are developed, the more marketable options will be explored, with the more promising ones having an impact on thin-film solar cells: nanophotonis therefore drives the vision of a new generation of photovoltaic devices.

Before I focus on the diffractive designs I pursued in my project, I will first give a brief overview of the main techniques in section 2.2. I then analyse the model of an ideal scattering structure in section 2.3, called Lambertian texture, which led me to introduce a figure-of-merit in section 2.4, called the light-trapping 
efficiency $(L T E)$, that can purely assess the performance of a light-trapping technique itself - irrespective of the material, fabrication method and technology used. Before I apply the LTE to state-of-the-art proposals to identify the most promising strategies, I will review the important milestones of light-trapping for photovoltaics in section 2.5 and finally conclude with some specific remarks regarding future trends in section 2.6.

\subsection{The main light-trapping approaches}

\subsubsection{Non-diffractive techniques}

Maximizing the surface-to-volume ratio is the most intuitive way of enhancing the probability of photon absorption. Silicon nanowires are examples for such light-trapping strategies, where the electronic transport properties may additionally benefit from a radial junction, as illustrated in Fig. 2.1. Solar cell devices with conversion efficiencies of around $12 \%$ were reported for a nanowire junction geometry $[15,16]$, whereas the use of nanowires as an anti-reflection texture for $300 \mu \mathrm{m}$ thick substrates enabled device efficiencies of $18 \%[17,18]$.

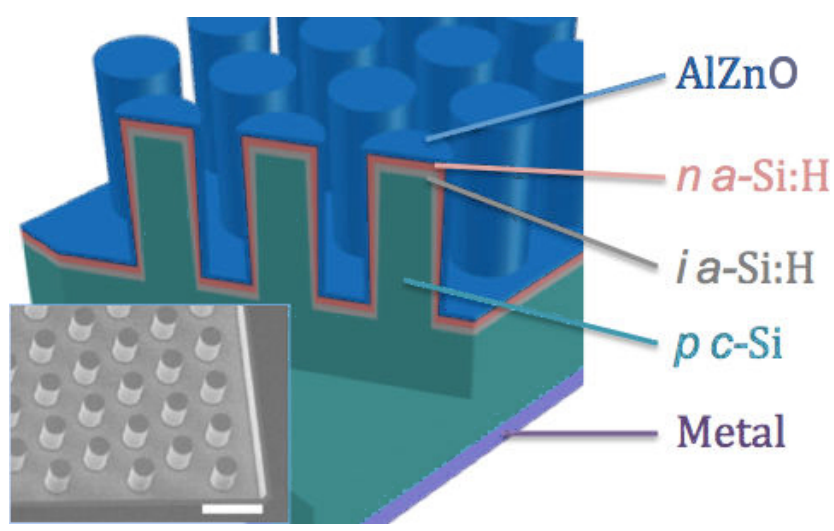

FIG. 2.1: Schematic of a Heterojunction with Intrinsic Thin Layer (HIT) radial junction silicon solar cell with $10 \%$ power conversion efficiency [19]. A $30^{\circ}$ tilted top-view image of a fabricated device is shown in the inset. The indicated scale bar as well as the pillar's length are $10 \mu \mathrm{m}$. 
However, too many research challenges still need to be addressed, before a commercial product based on nanowires can be introduced: mainly, the stability of the junction formation and the effective surface passivation, but also practical issues, such as the rapid scaling and the integration of nanowires into modules, need to be better understood and further improved.

Another method for enhancing the photon absorption takes advantage of the plasmonic resonance of metal nanoparticles. Metal nanoparticles can exhibit a very high polarizability at the resonance wavelength, and efficiently scatter the incident light into the thin-film. Here, the particle dimensions are a key parameter for the tuning of the resonance condition.

Solar cells with plasmonic scatterers have attracted a lot of attention [20], but the proposed effect turned out to be very sensitive to the material parameters. While enhancements of up to $30 \%$ in the power conversion efficiency were reported for plasmonic thin-film silicon solar cells, the absolute power conversion efficiency remained below $10 \%$ [21-25].

One of the issues I study later (see section 4.3) is the dissipative loss of such plasmonic structures; therefore, if the plasmonic scatterers were replaced by dielectric structures, the dissipative optical losses could be greatly reduced.

In fact, dielectric Mie resonators were recently proposed by Spinelli et al. [26]. The authors could demonstrate almost perfect impedance matching from the ultraviolet to the near-infrared spectral range using Si nanopillar arrays, i.e. less than $3 \%$ reflectivity was obtained over the entire spectral range $450-900 \mathrm{~nm}$ by $150 \mathrm{~nm}$ long nanowires with a $0.5 \mu \mathrm{m}$ array pitch and $60 \mathrm{~nm}$ thick $\mathrm{Si}_{3} \mathrm{~N}_{4}$ overlay coating.

To characterize the wavelength dependent scattering efficiency $Q_{s c a}$, the scattering cross section $\sigma_{s c a}$ is usually normalized to the geometrical area of the structure $\sigma_{\text {geom }}$. For dielectric Mie resonators, scattering efficiencies $Q_{\text {sca }}=\sigma_{\text {sca }} / \sigma_{\text {geom }}$ up to 10 have been demonstrated so far [26].

In addition, it has also been demonstrated that Mie-resonators could even be placed on top of an already completely finished thin-film solar cell for enhancing its power conversion efficiency [27]. 

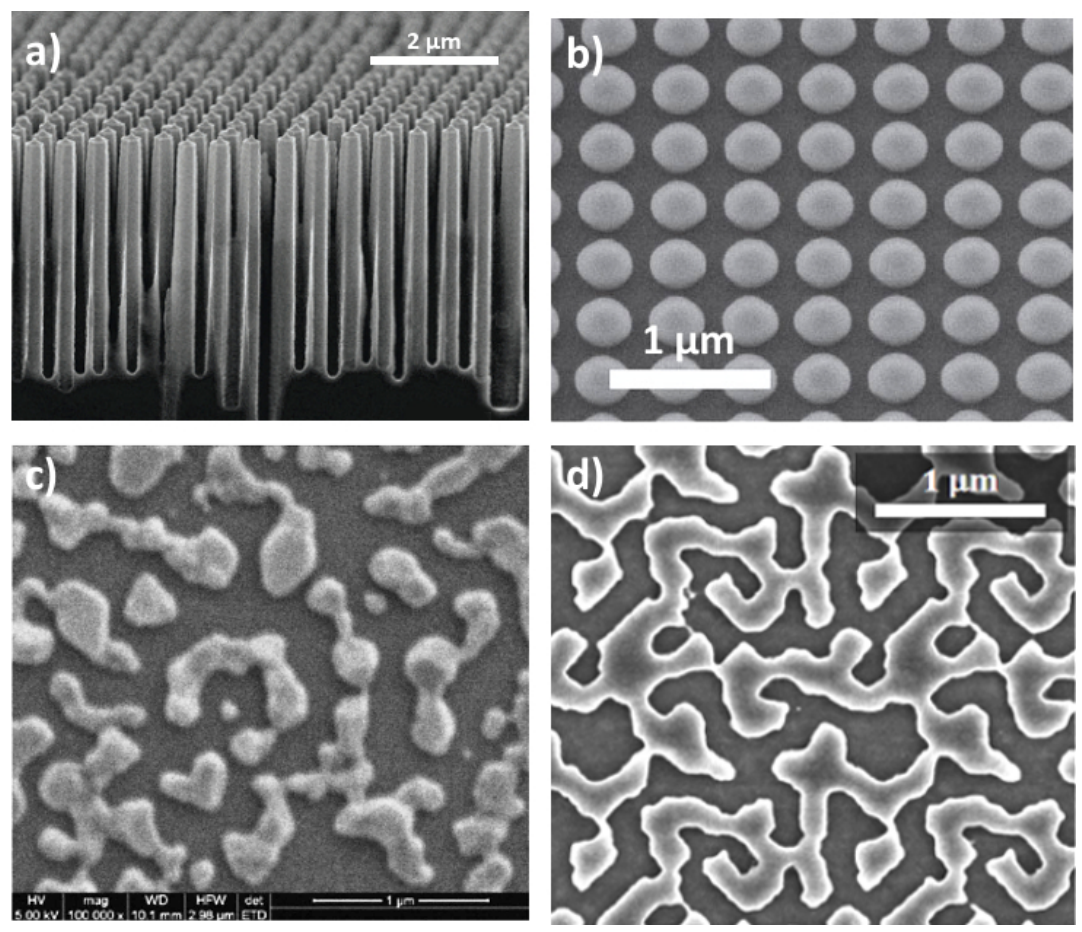

FIG. 2.2: Long silicon nanowires ( $4 \mu \mathrm{m}$ long) and arrays of small nanopillars $(150 \mathrm{~nm}$ high) have both shown to yield excellent anti-reflective properties. While the high-aspect ratio of closely spaced silicon nanowires (a) notably reduces the external back-reflection [28], dielectric Mie-scatterers (b) are able to strongly enhance the coupling efficiency of incident light into the absorber layer [26]. The Mie resonance of metallic nanostructures may in principal be superior to dielectric scatterers, given the high polarizability of localized plasmonic particles. In practice, however, the parasitic losses tend to almost compensate the benefit of the plasmonic scatterers. Please note that the best performing plasmonic structure in [29], here shown as image (c), looks quite similar to one of my own best performing diffractive textures, here shown as image (d), which made me wondering about the mechanism of the improved light trapping performance.

Thin and transparent dielectric coatings are a further and well-known antireflective technique. Commonly used materials are $\mathrm{SiO}_{x}, \mathrm{Si}_{3} \mathrm{~N}_{4}$, and some conductive oxides like $\mathrm{SnO}_{2}, \mathrm{ZnO}$ or tin-doped indium oxide (ITO) to name but a few [30]. Since the Fresnel-reflection

$$
\mathrm{R}=\left(\frac{n_{1}-n_{2}}{n_{1}+n_{2}}\right)^{2}
$$

strongly depends on the refractive index contrast of two bordering media with refractive indices $n_{1}$ an $n_{2}$, an intermediate 'buffer' layer can be used to reduce the reflection. For example, if a coating with refractive index $n_{A R}=\sqrt{n_{1} \cdot n_{2}}$ is 
chosen a quarter wavelength thick $\lambda / 4 n_{A R}$, the incident and reflected waves will largely cancel each other out by destructive interference. Anti-reflective coatings can thus be understood as optical-impedance matching layers at the interface between two bordering materials, e.g. air/semiconductor.

Ideally, the refractive index would vary continuously in the transition region from air to silicon, because one single coating can not meet the requirement for a broadband anti-reflective quality. Such an inhomogeneous refractive index profile is approximated by multiple coatings, increasing the refractive index stepwise. A triple layer system of $\mathrm{ZnS} / \mathrm{MgF}_{2} / \mathrm{SiO}_{2}$, for example, has shown to reduce the silicon reflectance down to $2 \%$ in the wavelength range from 440 to $960 \mathrm{~nm}$ [31]. However, the fabrication of such multilayer coatings is often too expensive for most commercial solar cells. In addition, the anti-reflection effect is very sensitive to the layer's thickness as well as to the angle of the incident light. For oblique incident light, the performance of thin film coatings can indeed dramatically change.

\subsubsection{Refractive approaches}

A varying refractive index at the interface of two bordering materials may be mimicked by a surface texture. The eye of a moth, for example, is inherently covered with sub-wavelength cone-shaped structures, helping moths to evade detection by predators and maximizing light capture for vision.

For wavelengths much larger than the typical feature sizes, the effect can be understood by a breakdown of the texture into multiple layers; the aspect-ratio thereby corresponds to the transition region from air to silicon. Approximately, and in analogy to anti-reflection multi-layer coatings, such textures can be described as a medium with an effective index stepwise increasing from air to silicon, as illustrated in Fig. 2.3a.

On the other hand, textures with dimensions of a few tens of micrometer can also reduce the external reflection of sunlight. If the wavelengths are much smaller than the structural feature sizes, radiation will generally bounce onto the textured surface multiple times (rather than out to the surrounding air), as illustrated in Fig. 2.3b. 
In addition, light changes its propagation direction when entering a different material, since the speed of light depends on the medium. The effect is called refraction (from the Latin word 'refringere') following the fact that an object partly in one medium and partly in another medium appears to be broken, e.g. a stick that is partly submersed in the water.

Refraction at surface textures therefore appears as a natural choice for enhancing the properties of a solar cell, because it affords the redirection of sunlight into the absorber layer without incurring additional losses. The surface of most commercial crystalline silicon solar cells are indeed textured with anti-reflective/refractive pyramids similar to those shown in Fig. 2.3c.

In conclusion, refractive textures in combination with sub-wavelength features (as those shown in Fig. 2.4) may enable efficient light-trapping action over a broad spectral and angular range.

On the basis of this idea, Han et al. recently outlined an economical way for suppressing the surface reflection of a polished silicon wafer from $30 \%$ to $3 \%$ between 400 and $900 \mathrm{~nm}$ wavelength [32], as illustrated in Fig. 2.4.
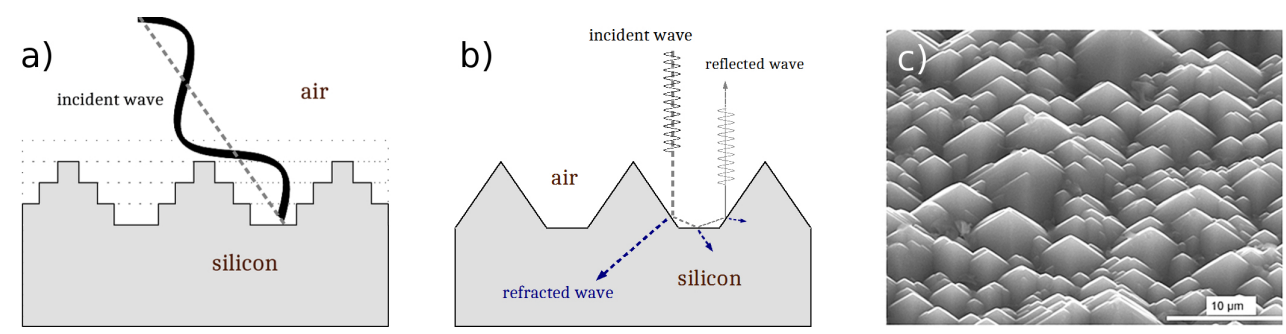

FIG. 2.3: (a) If the typical feature sizes are smaller than the incident wavelength, light will see the texture as an inhomogeneous medium with an effective refractive index gradually increasing from air to silicon. In analogy to a multilayer anti-reflection coating, this effect reduces the reflection dramatically. (b) If the typical feature sizes are larger than the incident wavelength, light will usually undergo multiple reflections on the textured surface, which in turn increases its probability of entering the absorber material. Since light is also refracted at the air-silicon interface, the path length through the absorber slab will be longer compared to the flat surface situation. (c) Large squarebased random pyramids often form the surface of an appropriately textured crystalline silicon solar cell [33], exploiting both anti-reflective and refractive effects in order to enhance sunlight absorption. 

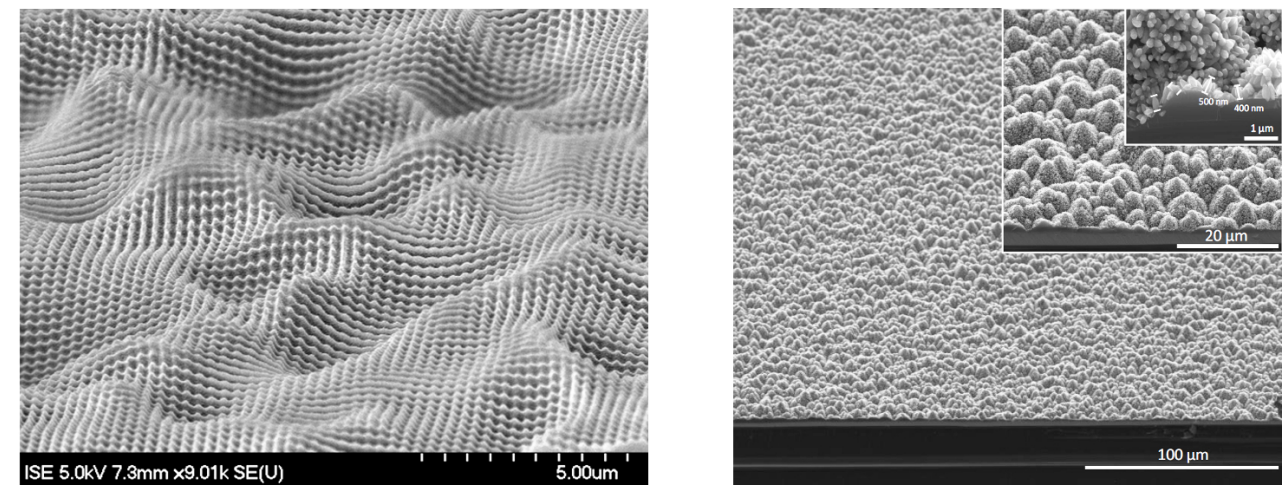

FIG. 2.4: Aperiodic refractive microstructures in combination with motheye sub-wavelength textures can provide excellent anti-reflection properties according to B. Bläsi [34] (left image) and S. Han et al. [32] (right image).

\subsubsection{Diffractive approaches}

Light is redirected when it bounces off a barrier (reflection) or goes from one medium to another (refraction). However, light may also change its propagation direction as it passes through an opening or around an obstacle in its path. Since the incident light thereby breaks up into different directions, the phenomenon is called diffraction (from the Latin word 'diffringere'). Consequently, light can be diffracted at a surface texture, if its ridges and growths are understood as the openings and obstacles.

Augustin-Jean Fresnel [35] was first able to explain diffraction as the resulting interference pattern of waves that were formed behind openings or obstacles. The superposition of all diffracted waves is commonly known as the diffraction pattern; mathematically, it is derived from a Fourier-transform of the diffracting structure.

In order to exploit diffractive effects, the typical structural sizes $\Lambda$ need to be similar to the incident wavelength $\lambda$ as illustrated in Fig. 2.5.

For example, radiowaves can bend around mountains in contrast to microwave (mobile phone) signals. Bat's echo calls are rather reflected than diffracted from their prey $(\lambda \ll \Lambda)$, whereas an object would be impossible to resolve when it is smaller than the wavelength with which it is observed $(\lambda \gg \Lambda)$. 
a)

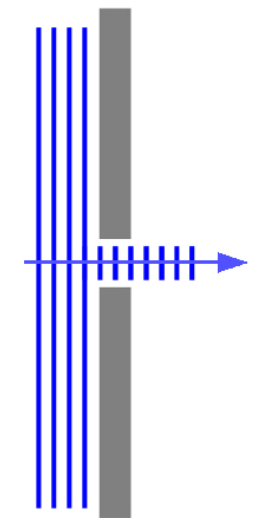

b)

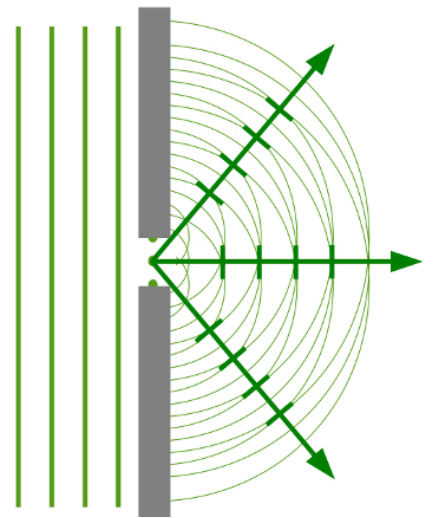

c)

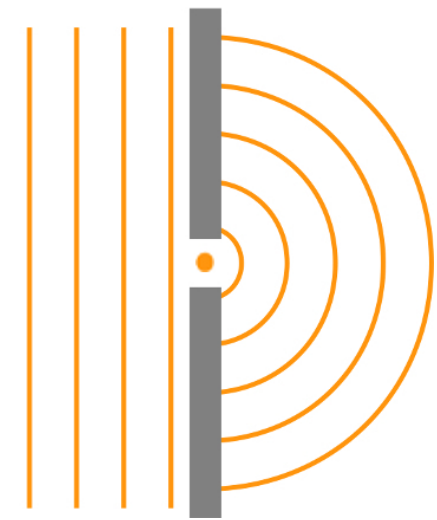

FIG. 2.5: The drawings illustrate three possible interactions for wavefronts of light - coming from a distant source - with the opening of a barrier. (a) If the wavelength is much smaller than the slit width, light propagation can be approximated by geometrical ray optics. (b) If the wavelength is of similar size than the slit width, light will interfere and form a diffraction pattern behind the slit. The wavefronts of the constructive interfering waves are indicated as small bars. (c) If the wavelength is much larger than the slit width, the slit is considered as a source of a new propagating wave behind the slit.

If the degree of diffraction depends on the relationship between $\lambda$ and $\Lambda$, white sunlight can split into its colours when it interacts with a diffractive structure. Ideally, a diffractive structure on the surface of a silicon solar cell would deflect the longer wavelengths into the plane of the silicon absorber, because those wavelenghts require absorption lengths often beyond the absorber thickness.

If the silicon slab is further embedded in a low-index material, like air, diffracted light may also be totally internally reflected back at the cladding. In this case, light is trapped in the plane of the silicon absorber layer - the key goal that light trapping tries to achieve. A thin-film silicon solar cell with a light-trapping nanostructure then behaves similarly to a waveguide and the nanostructure can be understood as the coupling interface for sunlight.

\subsubsection{The grating equation}

Since diffraction results in a change in direction, the incident photon needs to exchange momentum with the grating. The energy and momentum of the incident photon must thereby be conserved, while - in analogy to the crystal lattice momentum in a solid - the transfer of a grating momentum $G$ is restricted to 
integer multiples $m$ of $G$, which is directed parallel to the grating's surface:

$$
k \cdot \sin \theta_{i n}+m \cdot G=k \cdot \sin \theta_{m} \quad \text { with } G=\frac{2 \pi}{a}
$$

Here, $a$ is the spatial period of the grating structure and $\theta_{i n}$ and $\theta_{m}$ are the incident angle and diffraction angle of the $m$ th-order, respectively, as illustrated in Fig. 2.6. The angles are defined as positive when measured counter-clockwise. If $\lambda$ stands for the free-space wavelength of light, $k=2 \pi / \lambda$ for the wave-number and $n_{1}$ and $n_{2}$ are the wavelength dependent refractive indices of the incident and the propagating medium, respectively, rearranging the grating-equation explicitly highlights the dispersive character of a grating, because $\theta_{m}$ directly depends on the incident wavelength $\lambda$ :

$$
n_{1}(\lambda) \cdot \sin \theta_{i n}+m \cdot \frac{\lambda}{a}=n_{2}(\lambda) \cdot \sin \theta_{m}
$$

The grating-equation in this form would suggest to reconcile diffraction as an extension of Snell's refraction law, which is reproduced for $m=0$ in Eq. 2.3. This idea was indeed recently discussed by S. Larouche and D. Smith [36], who were able to establish a formal equivalence between generalized refraction and blazed diffraction gratings.

\subsubsection{Discussion}

By assuming normal incidence $\theta_{i n}=0$, solving the grating equation for the highest diffraction order

$$
|m| \leq \frac{a}{\lambda / n_{2}(\lambda)} \quad \text { for } \quad \theta_{m}=\pi / 2
$$

and for the diffraction angle $\theta_{m}$, where the bijection $\arcsin (\sin x)=x$ can be applied on $x=\theta_{m}$, because $\theta_{m}$ is in the interval $(-\pi / 2,+\pi / 2)$ for transmission into medium $n_{2}$,

$$
\theta_{m}=\arcsin \left(\frac{\lambda / n_{2}(\lambda)}{a / m}\right)
$$




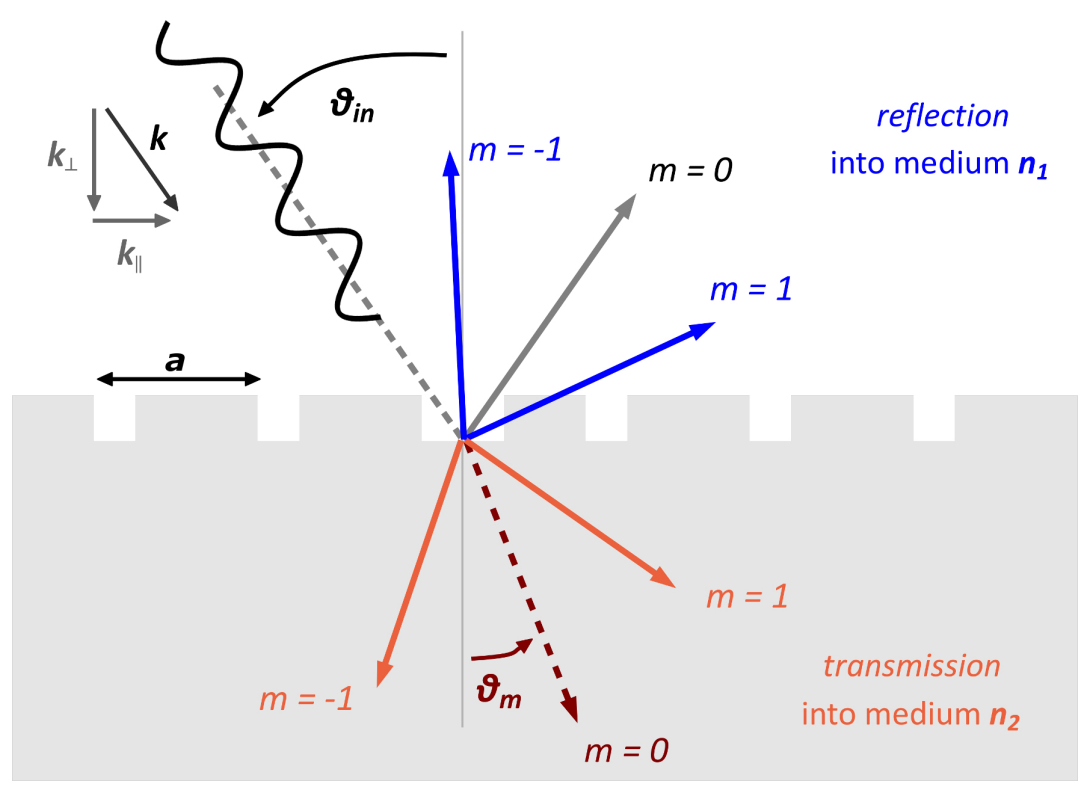

FIG. 2.6: Diffraction of an incident plane wave with wavelength $\lambda$ and wavenumber $k=2 \pi / \lambda$ at a structured interface layer between a medium with refractive index $n_{1}$ and medium with refractive index $n_{2}$. While $k$ remains conserved during the interaction, diffraction requires a transfer of momentum with the grating structure. The periodicity of the grating, however, restricts the momentum transfer to integer multiples $m$ of $2 \pi / a$ and to directions within the periodic corrugations $a$. The important correlation between the momentum of a wave and the periodicity of a system is often explained as Bloch-theorem in solid state physics textbooks [37] and photonic crystal books [38]. The higher diffraction orders are thus the result of a stronger interaction with the periodic structure.

allows to draw the following two conclusions: although the number of diffraction orders $m(\lambda)$ scales with the grating period $a$ (see Eq. 2.4), the diffraction angle $\theta_{m}(\lambda)$ drops for wavelengths $\lambda^{*}=\lambda / n_{2}$ smaller than $a$ (see Eq. 2.5). Therefore, the more diffraction orders exist, the more light is diffracted into smaller angles. In addition, each order only contains a small fraction of the total intensity, when the energy is spread over a continuum of diffraction orders. On the other hand, choosing periods equal to a few wavelengths $\lambda^{*}$ will result in more intensive, but only a few diffraction orders.

Since the lower diffraction orders tend to be more intense, a promising strategy for enhancing the absorption of light aims to shift the energy from the lower to the higher orders, as these are more likely to be totally (internally) reflected at the cladding of a silicon slab. No optical losses would result, because the energy is only redistributed amongst the diffraction orders. 
In effect, this strategy strongly relies on the ability to control the phase of interfering waves. Changing the duty cycle is one example of how the absence of a diffraction order can be achieved. For example, if the ridges are half (or a third) of the grating's period, the second (or third) order will be suppressed by destructive interference.

While diffractive effects are only of minor importance in commercial solar cells today, nanophotonic structures are expected to enter the market soon. According to J. Harris (Stanford University), advisory board member from Solexel, nanodome patterns or truncated pyramids (U.S. Patent No. 8853521) are now used in commercial $40 \mu \mathrm{m}$ thin-film silicon solar cells in order to obtain broadband and wide angle anti-reflection action. Solexel already announced the introduction of very lightweight and cheap solar modules for this year - with module efficiencies above $20 \%$.

\subsection{The Lambertian Scatterer}

In order to assess a light-trapping structure, the performance of a given design has to be benchmarked against a theoretical limit. In this section, I will analyze the model of an ideal scattering structure, called Lambertian texture, which led me to introduce the figure-of-merit described in subsection 2.4.2.

Light is reflected or refracted (or both) at a non-structured interface, whereas a structured interface may additionally scatter the radiation into higher angular directions.

The concept of "haze" is one possible way to assess the benefits of a structured surface, because the haze value quantifies the percentage of non-refracted light. The more light is scattered, the higher the haze value would be. Consequently, more light experiences a longer path through the absorber as opposed to the situation of a non-structured interface (for which the haze value is zero). Achieving high haze over a broad range of wavelengths is therefore a desirable objective for enhancing the absorption of a thin silicon slab. 
However, if the path lengths of scattered and refracted light do not differ by much, a high haze value may not necessarily translate into a higher absorption. Therefore, haze alone is not a sufficient requirement for characterising an effective light trapping structure.

A better parameter is path length enhancement: the greater the scattering angle $\theta$, the longer the path $\ell$ through the absorber. I can then define the scattering efficiency in terms of the path length enhancement $\ell / d$ with $d$ representing the slab thickness:

$$
\ell=d / \cos \theta=d \cdot \sec \theta
$$

The question of the maximum path length enhancement in a weakly absorbing slab was analyzed by E. Yablonovitch and G. Cody [39], who assumed the scattering layer to be isotropically radiating; such textures are called Lambertian, because their angular intensity distribution (AID) obeys Lambert's cosine law [40], see Fig. 2.7. The Lambertian Scatterer represents the perfect diffuser in the ray-optics approximation, with the radiated energy being understood to flow in straight lines.

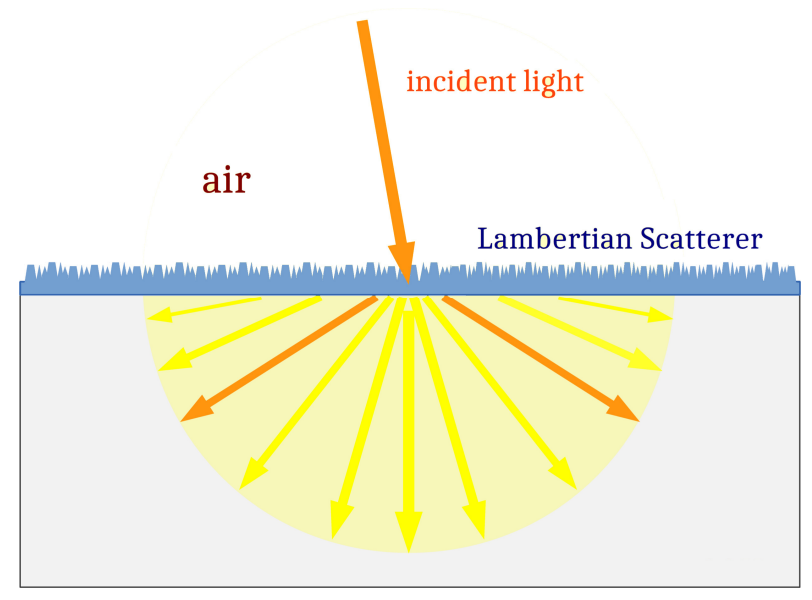

FIG. 2.7: If a scatterer radiates isotropically into a material, the light would be transmitted equally in all directions with a lobe in the shape of a hemisphere. The angular intensity distribution (AID) thereby obeyes Lambert's cosine law [40], whereas the energy will in average flow into the scattering angle $\bar{\vartheta}=\arccos \left(\frac{\cos 0^{\circ}+\cos 90^{\circ}}{2}\right)= \pm 60^{\circ}$ (indicated by the orange scattered rays) independently of the the incident light, c.f. Fig. 2.10 on page 33. 
The light path is defined in the framework of geometric optics. If the wavelength $\lambda^{*}$ becomes comparable to both the absorber thickness and typical feature size of the surface $\Lambda$, near-field phenomena will modify the diffracted waves. The Frauenhofer distance $d_{f}=2 \Lambda^{2} / \lambda^{*}$ is a parameter often used for distinguishing the near and far-field zones from each other. Since the thickness of silicon solar cells always exceeds $d_{f} \approx 2 \mu \mathrm{m}$ and $\lambda^{*} \sim \Lambda$ for sunlight, the path length enhancement remains a good parameter for characterizing the scattering efficiency of a surface structure.

In the following, I will derive the absorption and the maximum path length enhancement by a Lambertian Scatterer in a different way compared to Yablonovitch \& Cody, because the authors had focused only on the weak absorption regime. My derivation is based on the rarely cited idea of J. Gee [41], who had already proposed a very intuitive description of light-trapping in 1988. I generalize Gee's concept to the problem sketched in Fig. 2.8.

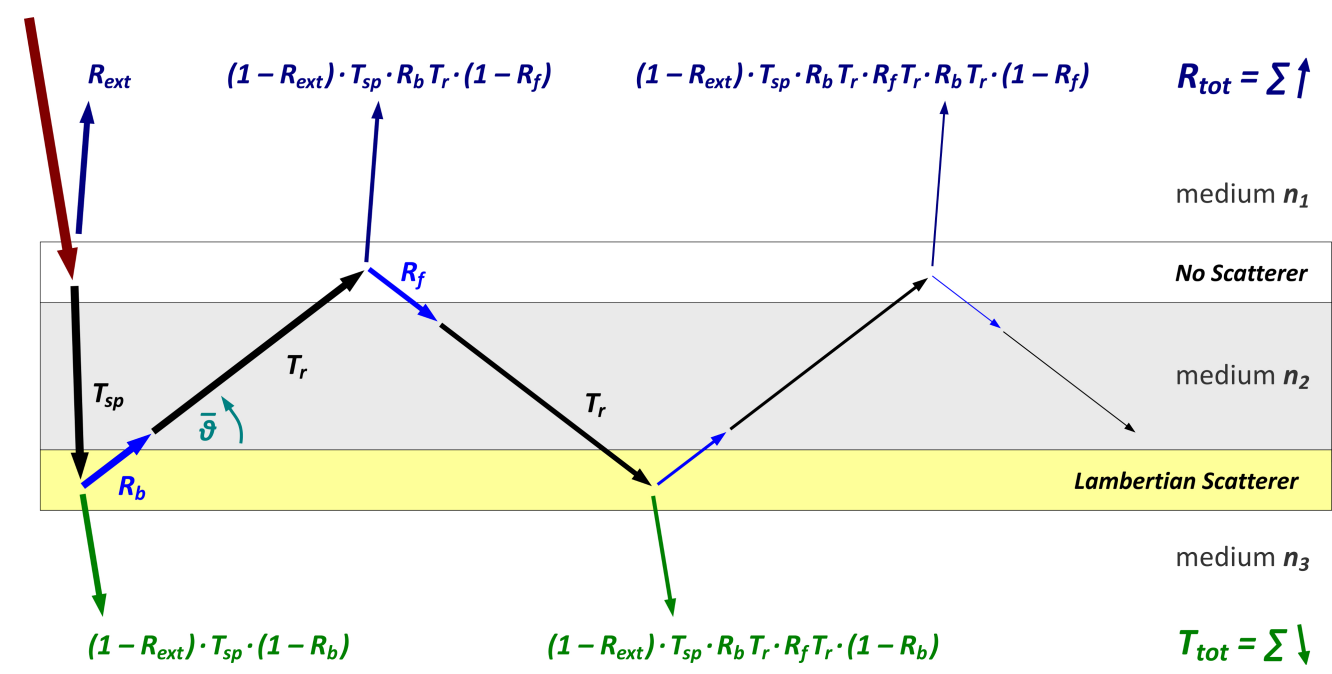

FIG. 2.8: Randomization of light at the scattering layer will allow us to neglect coherent effects, because a fixed phase relationship does not occur between diffusely-scattered, back-reflected and randomized light waves. The propagation of an averaged light ray in a lossy waveguide can then be described by the external reflection $R_{e x t}$, the internal effective reflectances $R_{b}$ and $R_{f}$ and the attenuated transmission $T_{r}$ with respect to the single-pass traversal $T_{s p}$. The total reflection $R_{t o t}$ into medium $n_{1}$ and total transmission $T_{t o t}$ into medium $n_{3}$ are therefore determined by the optical depth of the medium with refractive index $n_{2}$, the optical losses at the cladding layers and by the angleaveraged scattering efficiency. 


\subsubsection{Absorption enhancement by a Lambertian backscat- terer}

When the Lambertian scattering layer is situated on the rear side of a slab (as in Fig. 2.8), the calculation of the photon absorption is complicated by three issues:

1. before light arrives at the scatterer, some light may already have been reflected at the surface or absorbed during the first traversal through the slab,

2. for greater scattering angles $\theta$, less light will fall into the escape cone, defined by the critical angle $\theta_{c}$ of total internal reflection,

3. the angular intensity distribution (AID) follows Lambert's cosine law only if the slab becomes transparent.

I can cover the first issue by truncating the incident spectrum on the Lambertian texture, considering the external reflection air/Si and the absorption of the first traversal through the slab, as shown in Fig. 2.8.

By calculating the angle-averaged (internal) Fresnel reflection $R_{f}(\lambda)$ at the front interface, assuming rotational symmetry, I can effectively address the second point:

$$
R_{f}(\lambda) \equiv \frac{1}{\pi} \int R_{f}(\lambda, \theta) \vec{n} \cdot d \vec{\Omega}
$$

The more light is back-scattered from the surface normal $\vec{n}$ into the differential solid angle $d \vec{\Omega}$ of a hemisphere, the higher the effective front reflection will be. The normalization factor $1 / \pi$ is required by energy conservation, e.g. consider the case of an angular-independent reflectance $R_{f}(\theta)=$ const. :

$$
\begin{aligned}
R_{f}(\lambda) & \equiv \frac{1}{\pi} \int R_{f}(\lambda, \emptyset) \vec{n} \cdot d \vec{\Omega}=\frac{R_{f}(\lambda, \emptyset)}{\pi} \int|\vec{n}||d \vec{\Omega}| \cos \theta \\
& =\frac{R_{f}(\lambda)}{\pi} \int_{0}^{2 \pi} d \varphi \int_{0}^{\pi / 2} \cos \theta \cdot \sin \theta d \theta \\
& =R_{f}(\lambda) \cdot \int_{0}^{\pi / 2}(2 \sin \theta \cos \theta) d \theta=R_{f}(\lambda) \cdot\left[\sin ^{2} \theta\right]_{0}^{\pi / 2}=R_{f}(\lambda) .
\end{aligned}
$$


For a material with an high refractive index and small escape cone

$$
\begin{aligned}
R_{f}(\lambda) & \equiv \frac{1}{\pi} \int_{f} R_{f}(\lambda, \theta) \vec{n} \cdot d \vec{\Omega} \\
& =\int_{0}^{\pi / 2} R_{f}(\lambda, \theta)(2 \sin \theta \cos \theta) \cdot d \theta=\int_{0}^{\pi / 2} R_{f}(\lambda, \theta) \sin (2 \theta) \cdot d \theta \\
& =\int_{\theta_{c}}^{\pi / 2} \underbrace{R_{f}(\lambda, \theta)}_{=1} \sin (2 \theta) \cdot d \theta+\int_{0}^{\theta_{c}} R_{f}(\lambda, \theta) \sin (2 \theta) \cdot d \theta \\
& =1-\left(\sin \theta_{c}\right)^{2}+\int_{0}^{\theta_{c}} R_{f}(\lambda, \theta) \sin (2 \theta) \cdot d \theta,
\end{aligned}
$$

the last term may be negligible. For total internal reflection, $\sin \theta_{c}$ in Eq. 2.8 can be further replaced by the ratio of the refractive indices of the interfacing materials, according to Snell's refraction law Eq. 2.3 (for $m=0$ ).

The third point is easily violated in the presence of coherent effects (resonances), yet it is quite reasonable to assume incoherent scattering for a perfect diffuser. This assumption allows me to proceed with a statistical ray optics approach, where the light-paths are completely randomized and the phase information lost. As most backscattered light rays will differ in their path through the slab of thickness $d$, sometimes authors have used the angle-averaged path length $\bar{\ell}=$ $d \cdot \sec \bar{\theta}$ for the calculation of the randomized transmission $T_{r}$ :

$$
T_{r}(\alpha, \bar{\theta})=e^{-\alpha \bar{\ell}}=\left(T_{s p}\right)^{\sec \bar{\theta}}
$$

where $\alpha$ stands for the wavelength-dependent absorption coefficient and $T_{s p}$ for the transmission of a non-randomized single pass traversal through the slab. In the literature, the exponential factor $a=\sec \bar{\theta}$ is also known as the Lambertionality factor [42]. However, when I properly take into account that the radiance of each propagating light ray is reduced by a factor of $e^{-\alpha d \cdot \sec \theta}$

$$
T_{r}(\alpha d) \equiv \int_{0}^{\pi / 2} e^{-\alpha d \cdot \sec \theta} \sin (2 \theta) \cdot d \theta
$$


and set Eq. 2.9 equal to Eq. 2.10, I can express $\bar{\theta}$ as a function of the absorption coefficient:

$$
\begin{aligned}
T_{r}(\alpha d) & =\int_{0}^{\pi / 2} e^{-\alpha d \cdot \sec \theta} \sin (2 \theta) \cdot d \theta \stackrel{!}{=}\left(T_{s p}\right)^{\sec \bar{\theta}} \\
& \Rightarrow \frac{\ln T_{s p}}{\ln T_{r}}=\cos \bar{\theta} \geq 0 \quad \text { since }|\bar{\theta}| \leq 90^{\circ} \\
& \Rightarrow \bar{\theta}(\alpha(\lambda), d)=\arccos \frac{-\alpha(\lambda) d}{\ln \left(\int_{0}^{\pi / 2} e^{-\alpha(\lambda) d \cdot \sec \theta} \sin (2 \theta) \cdot d \theta\right)}
\end{aligned}
$$

Since the scattering angle $\bar{\theta}$ indicates the average flow direction of energy from the scatterer into the absorber slab as illustrated in Fig. 2.7, $\bar{\theta}$ depends on the absorber's material properties and thus can not be defined a priori to calculate the transmitted intensity via Eq. 2.9, i.e. $T_{r} \Rightarrow \bar{\theta}$.

For example, if all the incident energy were scattered into an angle $\bar{\theta}$, the angular intensity distribution (AID) of the Lambertian Scatterer would be replaced by a small lobe. The statistical nature of the Lambertian model will then be misinterpreted, because a Lambertian Scatterer spreads the incident light into a full hemisphere.

The graph in Fig. 2.9 now demonstrates that the average scattering angle of a Lambertian diffuser drops in the presence of absorption for shorter wavelengths; this drop is not due to imperfect randomization of the incident light by the scatterer, but due to the loss of isotropy of the scattered radiation due to absorption, which equally leads to a deviation from Lambert's cosine law.

In conclusion, the radiance of a Lambertian Scatterer becomes anisotropic in absorbing media, such that it would be misleading to assume the average-scattering angle $\bar{\theta}$ or the average path length $\bar{\ell}$ as independent of the material properties. From the point of view of scattering, a structure then should not be optimized for a thin absorber layer or for the weak absorption regime only, but for optical depths greater than $0.01 \mathrm{~dB}$. 


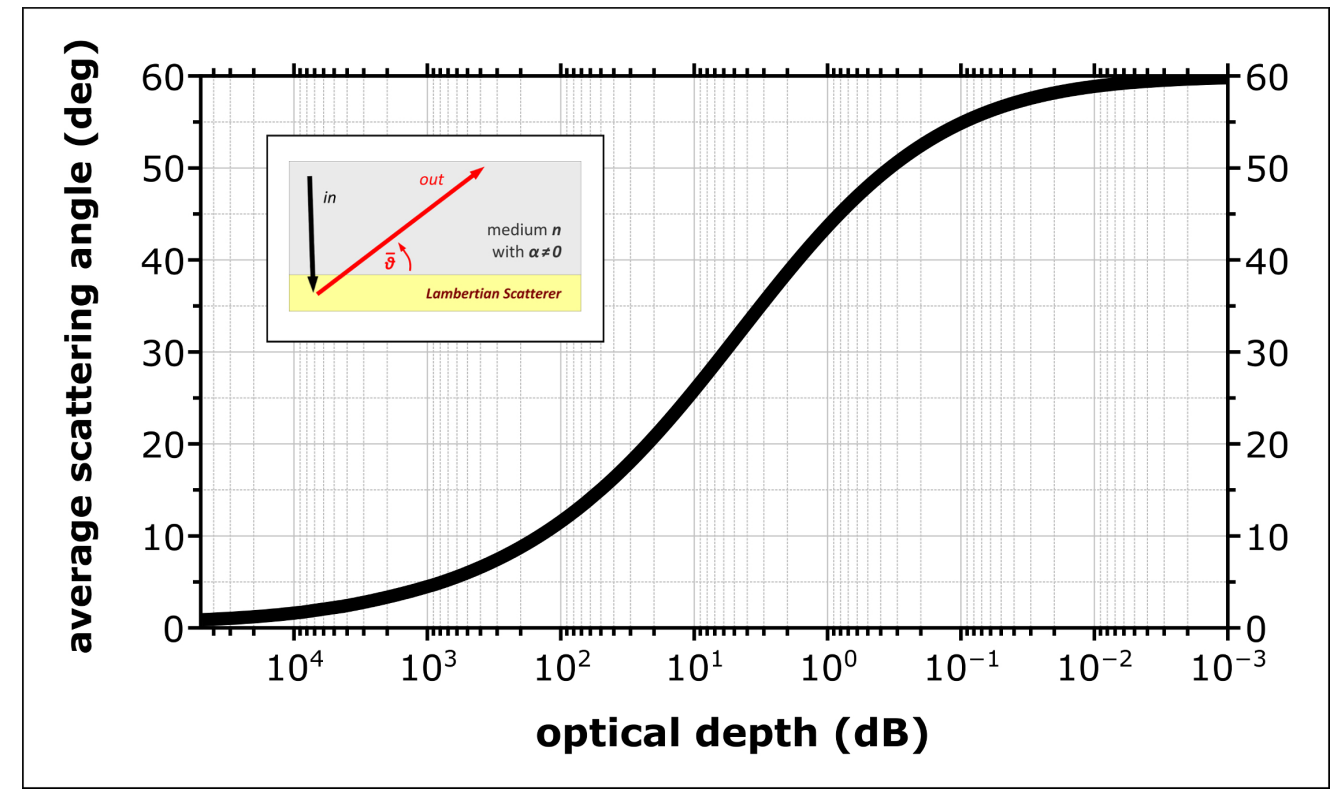

FIG. 2.9: The average scattering angle $\bar{\theta}$ specifies the average direction of the radiated energy flowing into a medium with refractive index $n$ and thickness $d$. The angular intensity distribution (AID) of a Lambertian diffuser, however, can deviate from the cosine law in presence of absorption $\alpha \neq 0$. The radiance then looses its isotropic character, because the energy flow is less attenuated for rays that are scattered into smaller angles. In subsection 1.3.1, I defined the optical depth of $0.01 \mathrm{~dB}$ as the medium's threshold value for transparency. A Lambertian Scatterer thus will scatter Lambertianly only, when the optical depth falls below $0.01 \mathrm{~dB}$, i.e. when the product of $\alpha$ and $d$ is smaller than 0.003 - which stands in contrast to a wavelength independent scattering angle $\bar{\theta}(X)$ or the constant Lambertionality factor $a(X)=\sec \bar{\theta}=$ const. by Battaglia et el. [42]. Please note that the Yablonovitch \& Cody treatment assumes a constant angle of $60^{\circ}$, so especially for strongly absorbing media, this angle may be very different form the effective angle shown here.

\subsubsection{The Lambertian Limit}

In the following I will derive the total absorption of a slab with a Lambertian Scatterer at the back or front side. Since the outlined derivation is based on the multiple reflections at the cladding layers, the only required quantities to know are a) the optical depth of the absorber slab and b) the refractive index contrast between the absorber and the incident or substrate medium. I will first calculate the absorption caused by the randomization of the light-paths through the slab, i.e. $T_{r}$, and afterwards account for the multipass nature of trapped light. The 
integral in Eq. 2.10 is evaluated with the help of the exponential integral

$$
\Gamma(\tau)=\int_{\tau}^{\infty} \frac{e^{-u}}{u} \cdot d u
$$

the substitution $u(\theta)=\tau / \cos \theta$ and two integrations by parts:

$$
\begin{aligned}
T_{r}(\tau \equiv \alpha d) & =\int_{0}^{\pi / 2} e^{-\tau / \cos \theta} \sin (2 \theta) \cdot d \theta=2 \tau^{2} \cdot \int_{\tau}^{\infty} \frac{e^{-u}}{u^{3}} \cdot d u \\
& =2 \tau^{2} \cdot\left(\frac{e^{-\tau}}{2 \tau^{2}}-\frac{1}{2} \int_{\tau}^{\infty} \frac{e^{-u}}{u^{2}} \cdot d u\right) \\
& =e^{-\tau}-\tau^{2} \cdot\left(\frac{e^{-\tau}}{\tau}-\Gamma(\tau)\right) \\
& =e^{-\tau}(1-\tau)+\tau^{2} \cdot \Gamma(\tau),
\end{aligned}
$$

and does only depend on the optical depth $\tau=\alpha d$, which is the product of the wavelength dependent absorption coefficient $\alpha$ and the physical thickness of the slab $d$. Finally, the total absorption $A_{t o t}$ of a slab with Lambertian back-scatterer can now be calculated by adding all reflected and transmitted light paths together like it was illustrated in Fig. 2.8:

$$
\begin{aligned}
A_{\text {tot }}= & 1-R_{\text {tot }}-T_{\text {tot }} \\
= & 1-\left(R_{e x t}+\sum_{m=0}^{\infty}\left(1-R_{e x t}\right) \cdot T_{s p} R_{b} T_{r} \cdot\left(R_{f} T_{r} R_{b} T_{r}\right)^{m} \cdot\left(1-R_{f}\right)\right) \\
& \quad-\left(\sum_{m=0}^{\infty}\left(1-R_{e x t}\right) \cdot T_{s p} \cdot\left(R_{f} T_{r} R_{b} T_{r}\right)^{m} \cdot\left(1-R_{b}\right)\right) \\
= & \left(1-R_{e x t}\right)-\frac{\left(1-R_{e x t}\right) \cdot T_{s p}}{1-R_{b} R_{f} T_{r}^{2}} \cdot\left[R_{b} T_{r} \cdot\left(1-R_{f}\right)+\left(1-R_{b}\right)\right] \\
= & \frac{\left(1-R_{e x t}\right)}{1-R_{b} R_{f} T_{r}^{2}} \cdot\left[\left(1-R_{b} R_{f} T_{r}^{2}\right)-T_{s p} R_{b} T_{r}\left(1-R_{f}\right)-T_{s p}\left(1-R_{b}\right)\right] \\
= & \frac{1-R_{e x t}}{1-R_{b} R_{f} T_{r}^{2}} \cdot\left[\left(1-T_{s p}\right)+\left(1-T_{r}\right) \cdot R_{b} T_{s p}+\left(T_{s p}-T_{r}\right) \cdot R_{b} R_{f} T_{r}\right] .
\end{aligned}
$$


$R_{\text {ext }}$ stands for the probability that an incident photon is reflected by the slab, whereas $T_{s p} \geq T_{r}$ is the transmitted fraction after the first direct traversal. The prefactor (recirculation factor) takes into account the attenuation of the average light ray due to the multiple upward and downward reflections at the cladding layers. The specular case is reproduced for $T_{r}=T_{s p}$, i.e. when the single pass absorption $\left(1-T_{s p}\right)$ is enhanced by the product of $R_{b}$ and $T_{s p}$. If the Lambertian Scatterer is designed to work in transmission, $T_{s p}$ must be replaced by $T_{r}$, which maximizes the total absorption $A_{t o t}$.

The effective backreflection $R_{b}$ is $100 \%$ only for a lossless reflecting surface and may otherwise be analogously defined to $R_{f}$ in Eq. 2.7:

$$
R_{b}(\lambda) \equiv \frac{1}{\pi} \int R_{b}(\lambda, \theta) \vec{n} \cdot d \vec{\Omega}
$$

The more light is back-scattered from the surface normal $\vec{n}$ into the differential solid angle $d \vec{\Omega}$ of a hemisphere, the higher the effective back reflection $R_{b}$ will be. A black substrate, for example, would reflect almost no incident radiation $\left(R_{b}=0\right)$ back into the absorber layer; a silver mirror is instead highly reflective $\left(R_{b}>95 \%\right)$ for wavelengths above $800 \mathrm{~nm}$, yet highly absorptive $\left(R_{b}<90 \%\right)$ for wavelengths below $500 \mathrm{~nm}$.

Since Eq. 2.14 and Eq. 2.7 allow us to calculate $R_{b}$ and $R_{f}$ for arbitray substrate and incident media, respectively, Eq. 2.13 can be used to calculate the absorption enhancement by a Lambertian Scatterer on the bottom or top (with $T_{s p}=T_{r}$ ) of absorber layers. 


\subsubsection{The maximum absorption enhancement}

The multipass nature of the Lambertian model is highlighted by the absorption enhancement due to the randomization of the path lengths. Dividing Eq. 2.13 by the absorption of a direct traversal $1-T_{s p}$ allows to quantify the enhanced absorption:

$$
\begin{aligned}
\frac{A_{t o t}}{1-T_{s p}}= & \frac{1-R_{e x t}}{1-R_{b} R_{f} T_{r}^{2}} \cdot\left[\left(\frac{1-T_{s p}}{1-T_{s p}}\right)+\left(\frac{1-T_{r}}{1-T_{s p}}\right) \cdot R_{b} T_{s p}+\right. \\
& \left.+\left(\frac{T_{s p}-1+1-T_{r}}{1-T_{s p}}\right) \cdot R_{b} R_{f} T_{r}\right] \\
= & \frac{1-R_{e x t}}{1-R_{b} R_{f} T_{r}^{2}} \cdot\left[1+\left(\frac{1-T_{r}}{1-T_{s p}}\right) \cdot R_{b} T_{s p}+\left(\frac{1-T_{r}}{1-T_{s p}}-1\right) \cdot R_{b} R_{f} T_{r}\right] \\
= & \frac{1-R_{e x t}}{1-R_{b} R_{f} T_{r}^{2}} \cdot\left[1+\eta \cdot R_{b} T_{s p}+(\eta-1) \cdot R_{b} R_{f} T_{r}\right] .
\end{aligned}
$$

Since $T_{r} \leq T_{s p}$ (see Eq. 2.12), the enhancement factor describing randomisation alone, which can be expressed as

$$
\eta(\tau \equiv \alpha d)=\frac{1-T_{r}}{1-T_{s p}}=1+\frac{\tau}{1-e^{-\tau}} \cdot\left[e^{-\tau}-\tau \cdot \Gamma(\tau)\right]
$$

is at best equal to 2 for weakly absorbed light $(\tau \rightarrow 0)$, while it is unity for strongly absorbed light $(\tau \rightarrow \infty)$, since the limit of the function $\tau \cdot \Gamma(\tau)$ is zero for both cases. The $4 n^{2}$ absorption enhancement limit derived in ref. [39] can then simply be reproduced in first order approximation for $R_{e x t}=0, R_{b}=1$ and $R_{f} \approx 1-1 / n^{2}$, with $n$ being the refractive index of the medium:

$$
\frac{A_{t o t}}{1-T_{s p}} \approx \frac{1-0}{1-\left(1-1 / n^{2}\right)} \cdot\left[1+2 \cdot 1+1 \cdot\left(1-1 / n^{2}\right)\right]=4 n^{2}-1
$$

The theoretical maximum absorption enhancement is reduced by the first direct traversal, because the $4 n^{2}$ limit corresponds to a Lambertian front-scatterer with $T_{r}$ replacing $T_{s p}$ in Eq. 2.13.

In reference to Eq. 1.1, I see light-trapping as the ability to increase the intensity by $2 n^{2}$ and the optical depth $\tau=\alpha d$ of a medium by two on average, at best:

$$
\left(2 \cdot n^{2}\right) \cdot(2 \cdot \tau)=4 n^{2} \cdot \alpha d
$$


Since the photon absorption is proportional to the incident photon flux, i.e. to the product of the photon density $\propto n^{3}$ and the group velocity $\propto c / n$, the enhancement of $n^{2}$ is due to the medium effect (brightness theorem).

The $n^{2}$ enhancement rests on the assumption of an equilibrium between the incident black-body illumination and the internal isotropic radiation field. For a perfect mirror at the backside of the thin-film, the enhancement factor is further increased by a factor of 2 , because the incident light intensity is virtually doubled. Scattering thus aims to maximize the optical depth $\tau$.

For a Lambertian scatterer, the average path length $\bar{\ell}$ through a slab is exactly twice the slab thickness:

$$
1-T_{r} \approx \alpha \cdot \bar{\ell}=\int_{0}^{\pi / 2}(\alpha d \cdot \sec \theta) \cdot \sin (2 \theta) \cdot d \theta=\alpha \cdot(2 d)
$$

as long as $\alpha d<0.01 \mathrm{~dB}$. Consequently, light is mostly scattered into the angle $\bar{\theta}=60^{\circ}$ in this weak absorptive regime, as illustrated in Fig. 2.10.

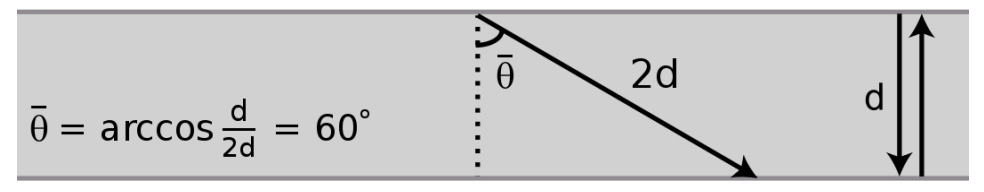

FIG. 2.10: If light is scattered into the angle $\bar{\theta}$ and traverses the absorber layer as it were twice as thick, the scattering angle will be $\bar{\theta}=60^{\circ}$.

If a scatterer is able to achieve $\bar{\ell}=2 d$ even in presence of absorption, it would outperform the Lambertian case, whereas a scatterer with $\bar{\ell}>2 d$ will beat the $4 n^{2}$ limit. Curiously, authors sometimes assume a Lambertian texture, but then consider all path lengths to be enhanced by $4 n^{2}$,

$$
A_{\max } \equiv 1-\exp \left(-\alpha \cdot 4 n^{2} d\right) \approx \frac{4 n^{2} \cdot \alpha d}{1+4 n^{2} \cdot \alpha d} \quad \text { for } \alpha d<0.01 \mathrm{~dB}
$$

which does misrepresent the physical picture. The comparison may still be adequate, as effectively the analogy was made between a textured slab and a $4 n^{2}$ times thicker unstructured absorber layer. However, $A_{\max }$ in Eq. 2.20 will overestimate $A_{t o t}$ in Eq. 2.13 for $\alpha d>0.01 \mathrm{~dB}$, i.e. when the angular intensity distribution (AID) falls below Lambert's cosine law. 


\subsubsection{Over the limits}

If interference effects violate the ray approximation, it becomes difficult to define an optical path length. The efficiency of a photonic structure must then be reformulated as its ability to enhance the optical depth of a thin film [43].

Assuming a silicon slab can be understood as an optical waveguide, Yu et al. [44] explain why the $4 n^{2}$ limit remains valid over a large spectral bandwidth.

The authors first compare the available number of propagating waveguide modes in each wavelength interval with the ability of a scatterer to couple sunlight in or out of these modes. The integrated absorption thereby serves as a figure of merit in this comparison, because a strong coupling is generally seen as incompatible with a large bandwidth. The outcome of the comparison then shows that the number of available modes directly scales with the absorber thickness, whereas the correlated coupling strengths are only subject to the design of the surface structure. Therefore, the maximum integrated absorption enhancement becomes independent of the absorber thickness when compared to the single-pass absorption.

Secondly, the authors demonstrate how a strong spectral dependency of periodic structures allows to achieve enhancement factors above $4 n^{2}$ in limited wavelength ranges. However, reducing the operating bandwidth does not maximize the integrated absorption, because the absorption would drop in other spectral regions. Finally, $\mathrm{Yu}$ et al. also highlight the effect of a strong angular dependency. If $\Theta_{\text {in }}$ is the limiting angle of incidence with respect to the normal to the surface, the coupling strength is proven to scale with $1 / \sin ^{2} \Theta_{i n}$. Absorption enhancement factors can therefore be as high as $4 n^{2} / \sin ^{2} \Theta_{i n}$, when the surface structure is designed to address only a selected range of incident angles. In fact, the $4 n^{2}$ limit was derived on the assumption of an acceptance cone covering the full hemisphere $\left(\Theta_{i n}=90^{\circ}\right)$, as mentioned in Fig. 2.7.

In conclusion, since efficient solar cell operation requires a broad angular and spectral response to sunlight, the $4 n^{2}$ absorption enhancement remains the limiting case for stationary solar cells. Higher enhancement factors may be possible in a limited wavelength range, but also reduce the total integrated absorption. 
Peters [45] was recently able to derive the same conclusion than Yu et al., yet his study only based on the conditions of how light is incident onto and escapes from an isotropic absorber. Therefore, the $4 n^{2}$ absorption enhancement factor is not a specific fundamental limit for diffractive light-trapping structures, but applies independently of the scattering mechanism.

\subsection{Assessment of light-trapping}

Many research proposals show large absorption enhancement factors or claim to have achieved the $4 n^{2}$ limit. However, the assessment of the light-trapping technique itself turns out to be difficult, because proposals often differ from each other either in the design or in the operating spectral and angular range.

In the end, I may get guidance from simulations of which geometries or features are desirable and from measurements indicating real performances. A quantitative assessment is further complicated by the vast diversity of methods used, which include anti-reflection and in-coupling concepts, 1D and 2D back-scatterers, embedded metal nanoparticles, photonic crystals and random or periodic diffractive structures on one or one both sides of the absorber layer. Since all of these methods are applied to different materials and layer thicknesses, it seems difficult to define a figure-of-merit that can purely assess the performance of a light-trapping technique - irrespective of the material, fabrication method and technology used.

\subsubsection{How to assess light-trapping structures for solar cells ?}

Light-trapping is commonly assessed by the absorption enhancement compared to a flat silicon slab, so a common figure-of-merit is the ratio of the absorption $A_{\text {max }}$ of a structured slab to the absorption $A_{\text {ref }}$ of an unstructured reference slab:

$$
F \equiv \frac{\int A_{\max }(\lambda) \cdot \mathrm{d} \lambda}{\int A_{\mathrm{ref}}(\lambda) \cdot \mathrm{d} \lambda}
$$


Since the enhancement factor $F$ is maximal in the weak-absorption regime, i.e. when the optical depth is in the order of some centi-dBs, many studies cited enhancements factor only for this regime. However, light-trapping for photovoltaic applications aims to increase the absorption over the full wavelength range of the solar spectrum, i.e. from $300 \mathrm{~nm}$ up to the wavelength bandgap $\lambda_{\max }$ of the absorber material, so such high enhancement factors have little practical utility. Instead, the quantity directly related to the integrated total absorption is the generated electrical current of a solar cell. If there was no voltage drop, the short-circuit current $J_{s c}$ would be the largest current which may be drawn from a solar cell. Therefore, light-trapping might be assessed in terms of the $J_{s c}$ enhancement compared to a non-structured reference:

$$
F^{*} \equiv \frac{J_{\max }}{J_{r e f}}=\frac{\int_{300 \mathrm{~nm}}^{\lambda_{\max }} A_{\max }(\lambda) \Phi(\lambda) \cdot \mathrm{d} \lambda}{\int_{300 \mathrm{~nm}}^{\lambda_{\max }} A_{\mathrm{ref}}(\lambda) \Phi(\lambda) \cdot \mathrm{d} \lambda} .
$$

Here, $\Phi$ is the photon flux of the standard reference spectrum AM1.5G, which is the solar spectrum most articles refer to.

However, the figure-of-merit $F^{*}$ would not distinguish between the causes of the current enhancement, because a higher enhancement factor $F^{*}$ could be achieved by a different anti-reflection coating or by a difference in absorber material quality.

In order to eliminate the material quality dependence, proposals have been benchmarked against the material dependent Lambertian enhancement factor $J_{L L} / J_{\text {ref }}$ :

$$
G \equiv \frac{F^{*}}{J_{L L} / J_{\text {ref }}}=\frac{J_{\max }}{J_{L L}},
$$

which expresses the performance of the proposed structure $J_{\max }$ against the theoretical achievement $J_{L L}$ of a Lambertian texture. Therefore, the $G$ factor quantifies the electrical current in percentages of an ideal Lambertian texture. 
For a few common photovoltaic materials, Bozzola et al. [43] reported the ideal short-circuit current $J_{\text {ref }}$ achieved with an unstructured slab against the theoretical maximum short-circuit current $J_{L L}$ achieved by a Lambertian texture as a function of the absorber thickness (exemplary shown in Fig. 2.11 for silicon). Their study graphically illustrates not only how the $G$ factor depends on the absorber thickness, but also why the $G$ factor rather impedes the assessment of light-trapping. For example, even a non-structured solar cell yields a $G>0$ or can approach $G=1$, because the current generated by only a double pass traversal of light can be as high as $40 \mathrm{~mA} / \mathrm{cm}^{2}$ in a $100 \mu \mathrm{m}$ thick silicon absorber layer, i.e. $G=40 / 45 \approx 0.9$ according to Fig. 2.11 .

Basch et al. [46] were the first to propose a thickness and material independent figure-of-merit that tried to isolate the light-trapping effect as much as possible. They suggested to compare the current gain by a proposed texture $J_{\text {max }}-J_{\text {ref }}$ against the theoretical gain by a Lambertian texture $J_{L L}-J_{\text {ref }}$ :

$$
G^{*} \equiv \frac{J_{m a x}-J_{r e f}}{J_{L L}-J_{r e f}}=\frac{G \cdot J_{L L}-J_{r e f}}{J_{L L}-J_{r e f}}
$$

If subtracting the current $J_{\text {ref }}$ of a non-structured solar cell from the current $J_{\max }$ of a structured solar cell removes the contribution provided by anti-reflection, $G^{*}$ would only quantify the benefits of the light-trapping technique.

However, the theoretical model of a Lambertian texture assumes a scattering layer of zero thickness. The anti-reflection effect of a Lambertian texture can thus take any arbitrary value. While ideal anti-reflection properties are generally assumed for the calculation of $J_{L L}$, practically, anti-reflection coatings will hardly cover the full solar spectrum. Therefore, if the denominator in Eq. 2.24 underestimates the anti-reflection properties of the reference cell, the $G^{*}$ factor will always remain smaller than 1 . In order to achieve a $G^{*}=1$, the proposed structure will need to either provide additional anti-reflection action apart from Lambertian light-trapping or it needs to outperform the performance of a Lambertian texture - the meaning of $G^{*}$ then remains ambiguous.

Pratesi et al. [47] defined a similar $G^{*}$ factor, but only suggested to replace $J_{L L}$ in the denominator of Eq. 2.24 with the total incident photon-flux of the sun 
$J_{\text {sun }}$ (expressed as an electrical current). This substitution would therefore not change any conclusions of the previous discussion regarding the format of the figure-of-merit, as the denominator will still not properly take the anti-reflection properties into account. In fact, the proposed figure-of-merit rather highlights the impossibility to achieve a $G^{*}=1$, because the current $J_{\max }$ of a structured solar cell would now need to absorb all incident photons from sun, whereas semiconductors are transparent to photons whose energies lie below their bandgap.

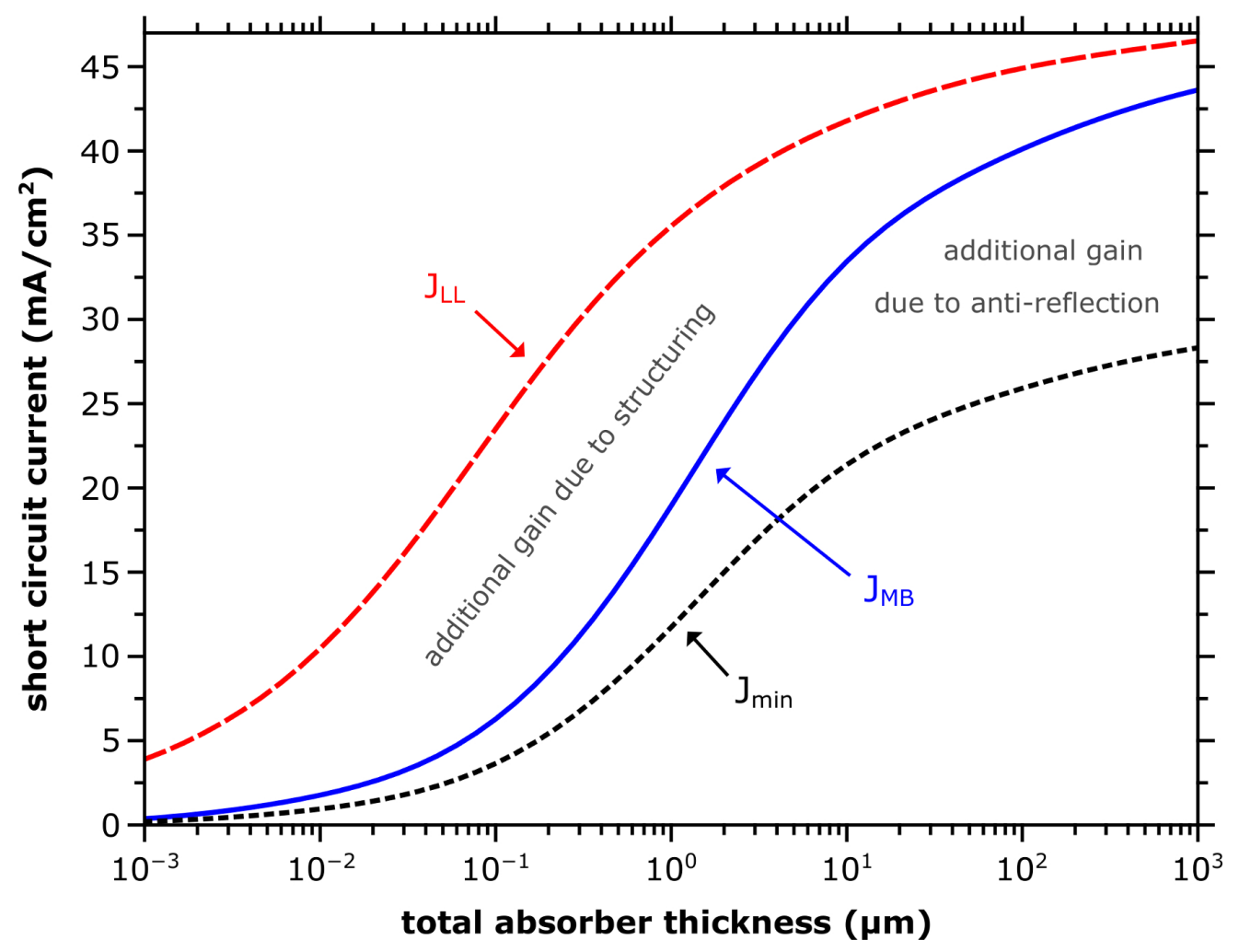

FIG. 2.11: In contemporary solar cell research, the figure of merit is often identified with the improvement in the short-circuit current. In the figure above, the short-circuit current density $J_{s c}$ for crystalline silicon is expressed as a function of the absorber layer thickness (assuming the AM1.5 global solar spectrum). The $J_{M B}$ and $J_{\min }$ graphs correspond to currents generated by a double pass traversal of light in the absorber layer with (blue solid) and without (black dotted) perfect anti-reflection coating, respectively. The $J_{L L}$ refers to devices textured with an ideal Lambertian scatterer and perfect anti-reflection coating (red-dashed line). All devices have a perfect mirror on the back. Please note that the red and the blue curves will converge for thicknesses greater than $10^{4} \mu \mathrm{m}$ due to the indirect nature of silicon's bandgap. For example, while the global solar irradiance is still more than $25 \%$ of its peak value at $1280 \mathrm{~nm}$ wavelength, a 1,000 $\mu \mathrm{m}$ thick silicon absorber does only absorb $20 \%$ of it by a double pass traversal of light, whereas a ten times thicker layer can already absorb $90 \%$ of it. 


\subsubsection{The light-trapping-efficiency (LTE)}

I proposed to replace $J_{r e f}$ in the denominator of Eq. 2.24 by the short-circuit current $J_{M B}$ of an ideal reference device.

$$
L T E \equiv \frac{J_{\max }-J_{r e f}}{J_{L L}-J_{M B}} .
$$

Since $J_{L L}$ and $J_{M B}$ assume perfect anti-reflection at the illuminated surface on the front and perfect reflection at an ideal metal mirror on the back of the absorber layer, the current gain $J_{L L}-J_{M B}$ purely reflects the light-trapping capability of a Lambertian texture. Therefore, the LTE separates the anti-reflection action from light-trapping, because it compares the real gain by structuring (nominator) to the hypothetical gain of ideal devices (denominator).

The format also follows the efficiency $\eta_{c}$ of a Carnot heat-engine, which compares the amount of entropy-free energy delivered to a load (nominator) against the ideal case (denominator) with the removal of all thermal energy $Q$ :

$$
\eta_{c}=\frac{Q_{\max }-Q_{r e f}}{Q_{\max }-0}
$$

In order to maximize the efficiency of a Carnot-engine, the heat $\operatorname{sink} Q_{r e f}$ in Eq. 2.26 would need to suspend all thermal motions and interactions. Correspondingly, in order to take advantage of light-trapping, $J_{\text {ref }}$ would need to be as close as possible to $J_{M B}$ in Eq. 2.25. Starting from a good reference device is thus as important as making a good light-trapping structure; otherwise, using a different anti-reflection coating layer could become more beneficial than lighttrapping.

The format of the LTE, however, seems to allow a higher figure-of-merit with the degree of worsening the reference device $J_{\text {ref }}$. Viewed optically, $J_{\text {ref }}$ will always consist of a coating layer, which could just be the encapsulation layer for protecting the solar cell from moisture, in addition to a back-reflector for enhancing the photon flux in the absorber medium. It is true, that both techniques, in reality, do not work over a broad bandwidth without any optical losses; anti-reflection 
coatings are not $100 \%$ transmissive as mirrors are not $100 \%$ reflective for all photonts. Therefore, once the optimized coating and back-reflector were applied, the same coating and mirror must also be applied to the structured solar cell device. Any reduction of $J_{\text {ref }}$ would then likewise affect $J_{\max }$, such that $J_{\max }-J_{\text {ref }}$ purely highlights the benefit of the structuring approach.

Secondly, since the material properties of $J_{\text {ref }}$ also determine $J_{M B}$ and $J_{L L}$, the $L T E$ remains independent of the material properties and only assesses the lighttrapping capability $J_{\max }$. A $L T E=1$ then means, that a structure achieved the same current gain as a Lambertian scatterer - irrespective of the method or technique used.

\subsubsection{The effective thickness}

The absorption in Eq. 2.13 was defined as a function of the total absorber layer thickness, assuming the Lambertian scatterer to be of vanishing thickness. Therefore, all calculated short-circuit currents shown in Fig. 2.11 refer to the total thickness $t_{t o t}$ of the silicon slab.

Since the absorption enhancement is also thickness dependent, I need to consider what thickness value to use for a structured material. Should I use the maximum thickness, i.e. the thickness between the bottom of the film and the peaks of the structure, or should I use some effective thickness $t_{\text {eff }}$ that represents the equivalent volume of material ?

The effective-thickness $t_{\text {eff }}$, which is the average thickness of the material, is often seen as a figure-of-merit for the greatest possible absorption achieved with the least volume of material. The most promising literature proposals might then be those with the smallest effective-thickness $t_{\text {eff }}$.

However, the effective-material-thickness $t_{\text {eff }}$ should not be confused with the effective-optical-thickness $\bar{\ell}$ defined by Eq. 2.9, which is the angle-averaged path length through the absorber; it is $t_{\text {eff }} \leq \bar{\ell} \leq 2 t_{\text {tot }}$ (see Eq. 2.19).

The LTE in Eq. 2.25 is defined for the total thickness $t_{t o t}$ of the absorber that includes the scattering layer, as was shown in Fig. 2.12. Therefore, the LTE does not take the material consumption into account. 
The effective material thickness seems to be a useful concept for comparing light trapping structures in terms of their material budget, but the performance of a scatterer depends not only on the volume of the absorber material $V_{S i}$ or on the geometry of the structure. The volume of material in between the structures $V_{c m}$ plays an equally important role, as it determines the refractive index contrast and thus the scattering efficiency. Since $t_{\text {eff }}$ always neglects the volume contribution of the complementary material $V_{c m}$, it consequently lowers the Lambertian limit of absorption:

$$
t_{e f f} \equiv \frac{V_{S i}}{A}<\frac{V_{S i}+V_{c m}}{A} \equiv t_{t o t}
$$

where $A$ stands for the illuminated surface area of the active absorber. To illustrate, if I was to construct an absorber layer of $1 \mu \mathrm{m}$ thickness with narrow pillars of $4 \mu \mathrm{m}$ height, the effective thickness might only be $t_{e f f}=1.2 \mu \mathrm{m}$, which makes for a very favourable comparison. Instead, I believe that one should use $t_{t o t}=5 \mu \mathrm{m}$, as the scattering layer adds $4 \mu \mathrm{m}$ to the absorber layer of $1 \mu \mathrm{m}$.

Ultimately, very narrow and high aspect-ratio structures, such as the long nanowires in Fig. 2.2a, mainly aim to maximize the surface-to-volume ratio. The higher the aspect-ratio, the larger the surface area and the more often a photon will interact with the structure, even though it may already entered and left the absorber material before - hence the higher its absorption probability for the same effective thickness $t_{e f f}$. The $L T E$, however, does not assess the interaction rate with the structure, but aims to assess the longer dwell time a photon spends in the absorber material due to a light-trapping structure, correctly addressed by the total absorber thickness $t_{t o t}$. 


\subsection{State of the art}

Before I will apply the LTE of Eq. 2.25 to literature proposals, a few important milestones that paved the way for thin-film silicon solar cell technology are reviewed. The survey in subsection 2.5.1 covers the important milestones that guided the advancement in our technical understanding. The current state-ofthe-art is summarized in subsection 2.5.2. Finally, as I was indeed able to identify a few structures very close to a light-trapping efficiency equal to $L T E=1$, I will analyze their common aspects in section 2.6.

\subsubsection{Important milestones}

Even if the genesis of photovoltaics can be traced back to the observations by E. Becquerel in 1839, progress in photovoltaics has been slow and has proceeded in approximately half-century steps.

The discretization of light in 1900 by M. Planck is often referred to as the foundation of quantum theory. The theory was not only able to explain why a metal plate can eject electrons under the action even very low intensity light (photoelectric effect), but also provided the framework for the realisation of the first silicon solar cell in 1946 by Ohl [48] and the point-contact transistor in 1947 by Shockley et al. [49].

In fact, the pioneering work by W. Shockley marked the invention of the pnjunction diode and the first practical silicon solar cell shortly after. Although

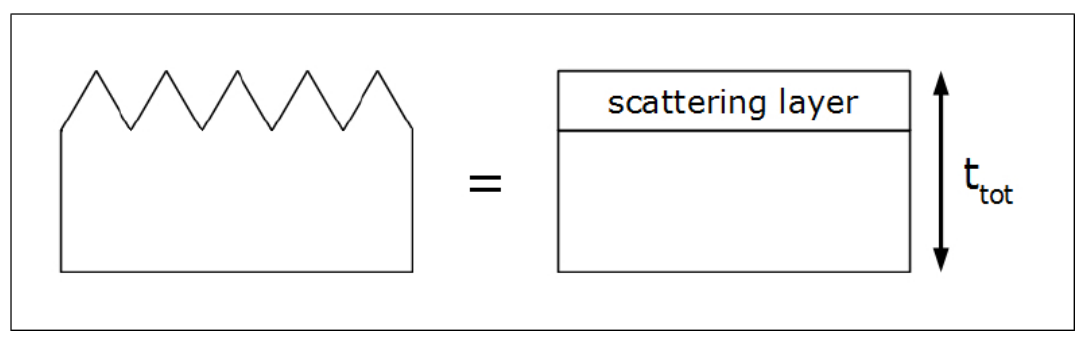

FIG. 2.12: The performance of a scatterer relies on the refractive index contrast and thus on the materials between the structures. Therefore, the LTE is defined for the total thickness $t_{t o t}$ of the absorber material that includes the scattering layer. 
the device was a milestone in the history of photovoltaics with a $6 \%$ power conversion efficiency, this success is only humbly reported in a very short letter by Chapin et al. in 1954 [50]. The reason may have been that Texas Instruments was already in the leverage position to manufacture silicon pn-junctions in volume [51], owing to G. Teal's technical achievement of silicon purification and crystal growth [52].

By the end of the 50s, silicon thus began to replace the industry's preferred semiconductor material, germanium, yet it took another half-century for a silicon photovoltaic industry to emerge.

The oil crisis of the 70s kick-started interest in solar power for terrestrial use. Competing with a large solar program in the USA, M. A. Green started the solar laboratory at the University of New South Wales in 1974. His research team soon became internationally recognized as one of the elite in the field; the group frequently set new world-record efficiencies by improved electrical and optical designs. For example, the first $20 \%$ efficient cell was fabricated by the group in 1985 [53] and was textured with a 1D periodic slanted surface as apparent from Fig. 2.13, allowing both improved light-in coupling at oblique angles and a reduced electrical transport resistance.

The benefit of such anti-reflective v-shape textures were realized in the mid 70s [54] and also assisted in the demonstration of $14-17 \%$ efficient but $400 \mu \mathrm{m}$ thick silicon solar cells in 1977 [55].

I would describe the mid 80 s as the foundation period for light-trapping in thinfilm solar cells, when E. Yablonovitch and G. Cody highlighted the importance of random textures as a means to trap the light in the absorber slab [39]. The authors also explained how light-trapping allows to increase the optical absorption of a semiconductor by $4 n^{2}$, if $n$ is the index of refraction. Shortly thereafter, Sheng et al. [56] demonstrated that periodic textures can even exceed this limit in the weak absorption region, as discussed in subsubsection 2.3.2.1. 


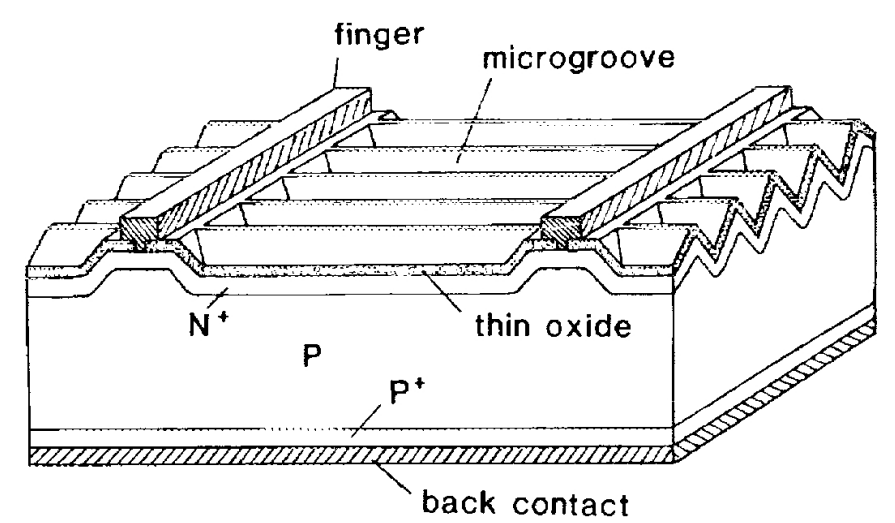

FIG. 2.13: The first $20 \%$ efficient silicon solar cells present microgrooved surfaces [53]. The slats of $5 \mu \mathrm{m}$ depth on a $10 \mu \mathrm{m}$ pitch not only retain the refractive and anti-reflective advantages of pyramidally textured surfaces, but also avoid electrical transport resistances for lateral current flow to the collecting fingers. The finger metallization of the cell is further restricted to regions which have not been grooved.

Deckman et al. [57] were the first who experimentally demonstrated absorption enhancement factors of up to 12. The same authors also showed that the achievements were directly translated into $25 \%$ higher short-circuit currents [58], though extremely thick a-Si:H absorber layers around $1 \mu \mathrm{m}$ were used in both studies.

P. Campbell and M. A. Green then tried to determine the optical performance of pyramidal textures with respect to anti-reflection and light-trapping action [59]. The authors found the textures to yield high anti-reflection and refractive lighttrapping, when the square based pyramids were at the front and a flat mirror at the back. The team later used such a texture to achieve a $24 \%$ efficient silicon solar cell with $280 \mu \mathrm{m}$ thickness [60].

The authors also recognized that the $4 n^{2}$ limit would only be achieved if the previously mentioned 1D slanted surface texture in Fig. 2.13 was kept at the front and the same pattern applied to the rear side of the absorber, yet perpendicularly aligned with respect to the top one. For a silicon solar cell of about $100 \mu \mathrm{m}$ thickness, such an arrangement would outperform pyramidal structures on both sides by $4 \%$ relative advantage in current generating according to their analysis [59].

At the end of the 80s, E. Yablonovitch provided further more background information and insights into the properties of a radiation field in a semiconductor 
[61]. Yablonovitch particularly focused on the consequences of artificially modifying the semiconductor with periodic structures. His paper about the "inhibited spontaneous emission in solid-state physics and electronics" is now recognized as one of the most important contributions to the field of photonics with more than 10,000 citations.

Contrary to intuition, he described light-trapping as the equivalence of restricting the spontaneous emission - which is the reversible process of photon absorption - into only those waveguide modes that are able to outcouple light from the material slab. The efficient operation of a solar cell does indeed require the reemission of most absorbed photons back to the sun, because the electrical voltage increases with the re-emission rate. However, while the internal emission would be lost, the externally incident radiation becomes internally trapped, permitting the semiconductor slab to be correspondingly thinner - at the same degree of absorption. Yablonovitch thus interprets light-trapping and the reduction of semiconductor volume as a consequence of restricting the spontaneous emission into only the essential waveguide modes.

The 90s are then characterized by the expanding market of silicon solar cells. Germany and Japan are today seen as case studies for a government-driven PV market, because the two governments had initiated subsidy programs in order to spur adoption, e.g. they were the first to introduce feed-in tariffs (1990).

From the scientific perspective, the major focus was the development of new, large-scale and low-cost manufacturing techniques [62]. Chou et al. introduced the imprint lithography technique with $25 \mathrm{~nm}$ resolution [63], for example, whereas Gombert et al. [64] demonstrated moth-eye structures that were fabricateable on a large-scale. Two long-lasting record cells were also made at this time.

The first record was made by the Japanese company Kaneka [65]. They realized a device utilizing a natural surface texture for the absorption enhancement of a $2 \mu \mathrm{m}$ thick polycrystalline silicon layer by a rough back reflector (STAR). The STAR-cell was not only 100 times thinner than the first practical silicon solar cell had been [50], the $10.7 \%$ efficiency was also almost twice as high as the original device and demonstrated that thin crystalline cells can achieve sufficient photon absorption by minute textured structures. The 90s may then also referred to as 
the decade of preparation for thin-film PV technology.

The second outstanding solar cell device was the PERL cell made by Zhao et al. [66] of Martin Green's group at UNSW, who were able to improve their former result by $2 \%$ through reduced surface recombination losses. The use of a thin, thermally grown $\mathrm{SiO}_{2}$ layer of about $20 \mathrm{~nm}$ thickness was found to effectively passivate the surface defects and allowed to establish the new performance level of $24.4 \%$ efficiency in 1998 . The device remained one of the top terrestrial silicon solar cells until 2013 [67]. Since the researchers were motivated by the fact that one third of the commercial PV products were based on large-grained multicrystalline silicon wafers (due to the lower costs), in the same mentioned study, the authors also showed a $6 \%$ improvement in the efficiency of multicrystalline solar cells. 
For multicrystalline silicon, anisotropic alkaline etching results in pyramids with completely random facets due to the uneven etch rates of the crystal planes [68]. The reflectance then becomes similar to an untextured surface according to J. Hylton [69]. The preferred texturing method for multicrystalline cells is therefore a wet isotropic acidic etching process [70].

Based on these observations, Zhao et al. [66] was able to texture the surface of a $260 \mu \mathrm{m}$ thick multicrystalline wafer from Eurosolare with the honeycomb structure shown in Fig. 2.14, which enabled to obtain a $19.8 \%$ power conversion efficiency. The authors showed that the refractive light-trapping properties of the hexagonal pattern were comparably effective to the inverted-pyramids used in monocrystalline silicon solar cells, but less efficient in terms of their antireflection action.

At the same time as M. A. Green published the intrinsic material function for crystalline silicon [11], Moharam [71], Whittaker [72] and co-authors outlined how the performance of light-trapping structures can be accurately simulated. These three papers provided the important groundwork for the emerging theoretical modelling period of diffractive light-trapping structures.
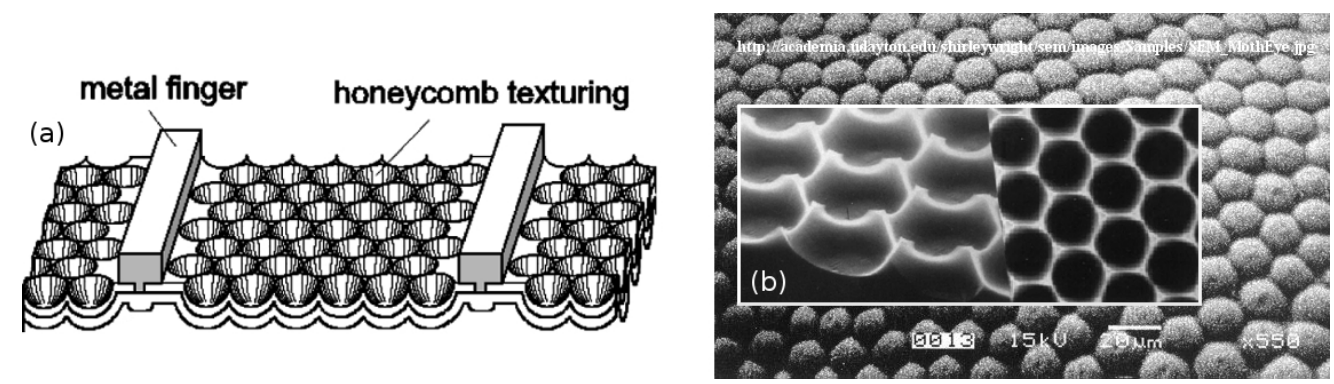

FIG. 2.14: (a) Schematic of a honeycomb surface texture for light-trapping. The texture could increase the optical path length of weakly absorbed light at $1060 \mathrm{~nm}$ wavelength by factors as high as 40 compared to a planar reference cell according to the authors [66]. However, such high enhancement factors are clearly not possible, because of the increased absorption in the rear metal reflector due to multiple light passes across the cell, which I will study later (section 4.3) as one of the key issues of metals. (b) The SEM images show the perspective view (left) and a plan view (right) of a fabricated honeycomb surface structure on a large-grained polycrystalline silicon wafer. The spacing of the hexagons is $14 \mu \mathrm{m}$. Since the texture resembles a reversed moth-eye pattern, the background picture was inserted to highlight this similarity. The protuberances inside the combs - not present in the honeycomb texture - are responsible for the excellent anti-reflection properties. 
I see Shah's article in Science [73] both as the end of the long childhood of photovoltaics and as the begin of a thin-film silicon solar cell technology, because his review about the recent developments of photovoltaic solar modules concludes with the suggestion that thin-film crystalline silicon is becoming the prime candidate for future photovoltaics.

After the turn of the millenium, photovoltaics became a popular research subject, which is reflected by the number of publications in Fig. 2.15 and by the citation history of the paper from W. Shockley and H. J. Queisser [13]. According to W. Marx [14], the analysis by Shockley and Queisser has remained unnoticed for about 50 years, before the study literally attracted attention: the paper occupies the citation-rank number 12 of all 11,723 physics papers entered in the Web of Science in 1961, and it attracted half of all citations from solar cell or photovoltaic papers for a 1961 paper [14].

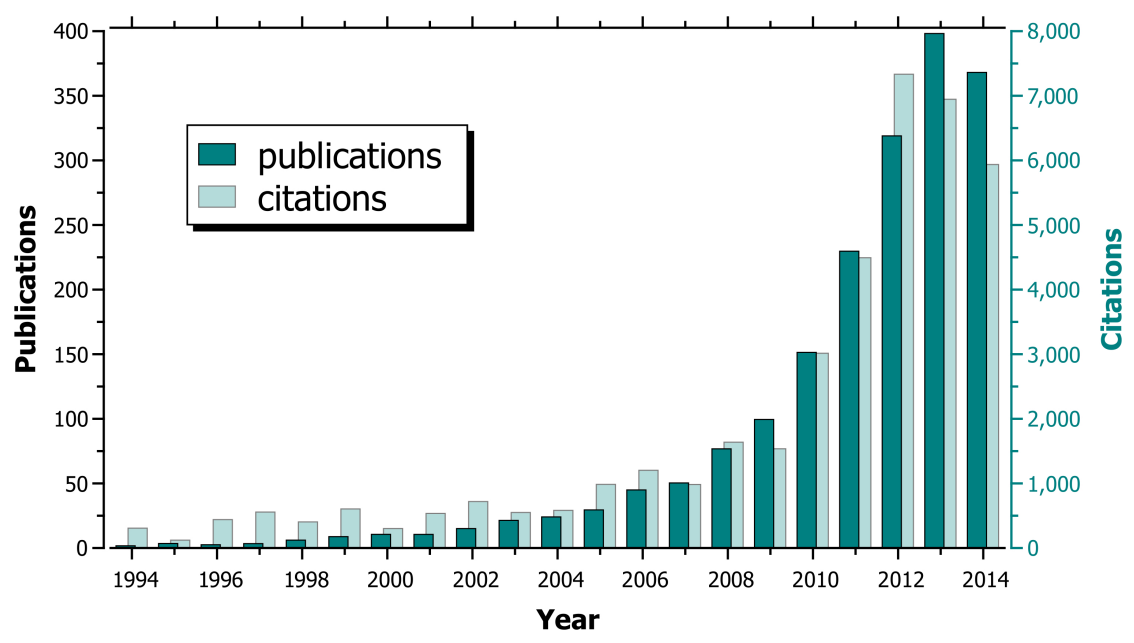

FIG. 2.15: Bibliographic analysis of publications (left) and citations (right) entered in the Web of Science (Thomson Reuters) before the 21st of November 2014. I used the keywords "light", "trapping" and "solar" and restricted the research areas to physics and optics. The total number of all publications is 2,000, whereas the number of all cited references is 38,000 in total. The exponential increase of research interest runs parallel with the steep increase of the cumulative PV capacity (not shown here). The silicon shortage between 2005 and 2008 could even have stimulated and intensified the scientific interest for light-trapping. For example, in 2005 a new article was published almost every week, while it ran up to one paper per day in 2012. Today, novel concepts, new strategies and emerging technologies in the PV field - like building-integrated, organic, dye-sensitized, plasmonic, CZTS, multi-junction, perovskite, HIT, flexible, hot-carrier, photon conversion, graphene, pentacene, quantum-dot or nanowire solar cells - make it easy to lose perspective. 


\subsubsection{The state of the art}

Light-trapping by random scattering (see Fig. 2.16) has since become the industrial standard for thin-film silicon modules [74]. At the same time, many novel methods for creating light scattering were introduced.

Metallic, dielectric 1D, 2D or 3D textures and photonic crystals were widely studied for their suitability for solar cells. The design optimization generally concentrated on the optical properties at the front (light in-coupling) or at the rear side (off-specular reflectance) of the thin-film absorber material. Structuring the back side normally addresses the red part of the spectrum only, since the blue and the green part of the visible spectrum are absorbed within the first few hundreds of nanometers in silicon. Therefore, patterning the absorber layer from both-sides increases the degrees of freedom available to the designer, as anti-reflective (front side) and diffractive functions (back side) can be combined $[75-77]$.
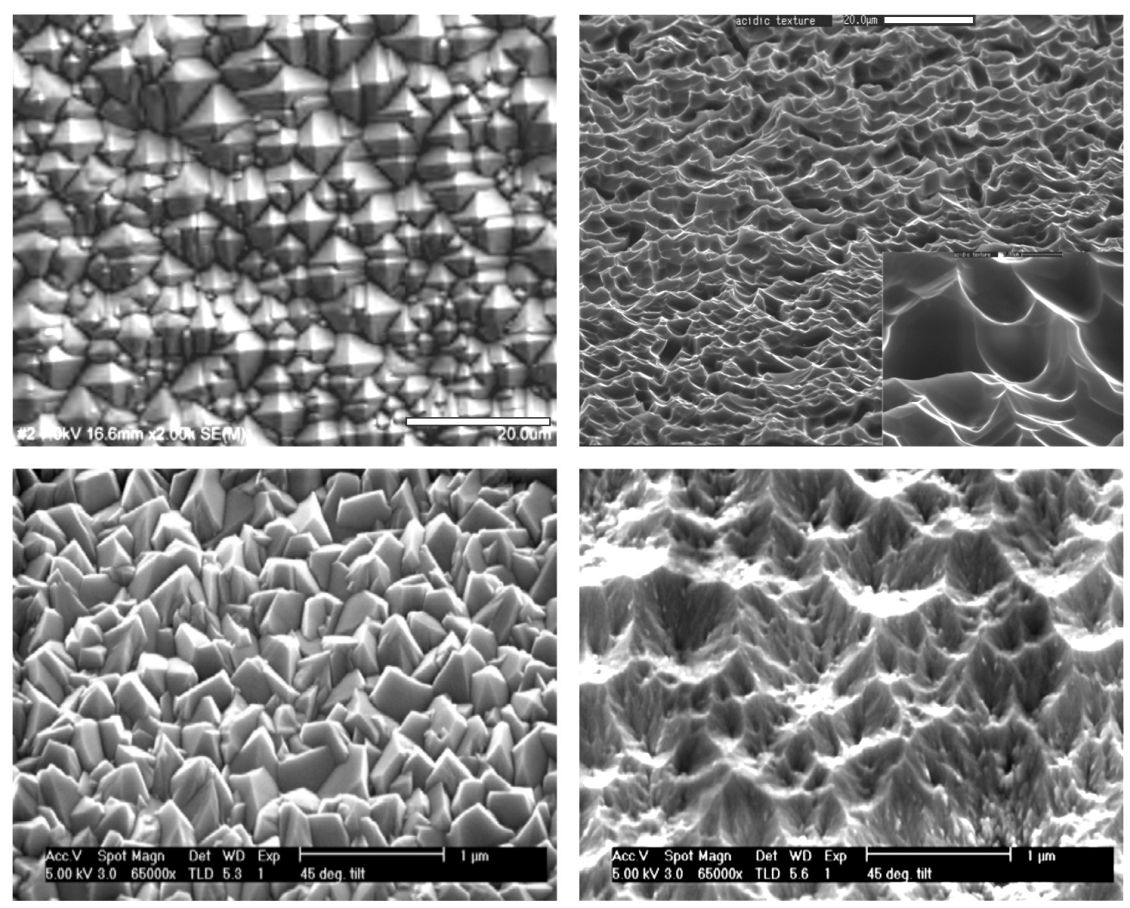

FIG. 2.16: Random surface textures increase the proportion of oblique scattered light into the absorber. Geometric light-trapping structures can easily be obtained in monocrystalline [78] (top left) or multicrystalline silicon [79] (top right) by chemical wet etching. Submicron light-trapping structures can form during the deposition, e.g. Asahi-U (bottom left), or may be chemically etched, e.g. into $\mathrm{ZnO}$ (bottom right) [80]. 
However, the advantages of using the strong scattering ability of metallic back reflectors are compromised by the substantial absorption losses in the metal itself. In the weak absorption limit, i.e. when light trapping is most beneficial, the light will interact even more often, so light-trapping, in fact, enhances parasitic absorption effects further. Campa et al. [81], for example, have found an overall reduction in the total reflectance of up to $40 \%$ in comparison to a specular silver reflector.

Such optical losses may, in principle, be minimized when the metal is separated from the silicon by a transparent oxide layer [82], as obliquely incident light will likely be totally internally reflected at a silicon/dielectric interface. Conducting oxide spacer layers between the metal and silicon absorber material indeed became a standard in state-of-the-art light-trapping designs. Thin conductive oxides thus can play a significant role not only as transparent front electrode, but also in improving the back reflection properties [83]. Alternatively, the metal mirror at the back can be replaced with a distributed Bragg reflector $[84,85]$.

In order to identify the most promising light-trapping strategies, I applied the LTE defined in Eq. 2.25 to numerous structures found in the literature.

While the majority of proposals are numerical simulations based on (intrinsic) c-Si, experimental studies are often conducted on hydrogenated microcrystalline silicon ( $\mu$-Si:H) layers. Although the absorption coefficient of $\mu$-Si:H depends on the defect density, crystalline/amorphous fraction and material morphology [86], its optical characteristic bears more similarity to c-Si than to a-Si:H. The LTEs of experimental structures for $\mu$-Si:H absorber layers were thus qualitatively assessed with the optical constants of c-Si.

When the experimental reference was not provided for the total thickness, I decided to use the ideal short-circuit current as the unstructured reference in order to establish a minimum for the light trapping performance.

Unfortunately, designs based on amorphous silicon were excluded from the analysis, because of the large variations found in the optical material properties: the material absorption of hydrogenated amorphous silicon (a-Si:H) strongly depends on the hydrogen concentration and on deposition-conditions, and these were often not provided. For example, the optical band gap of a-Si:H substantially varies between $1.4 \mathrm{eV}$ [87] and $2 \mathrm{eV}$ [88]. Therefore, the literature analysis was applied to crystalline silicon (c-Si) where well-known optical properties are available [11]. 


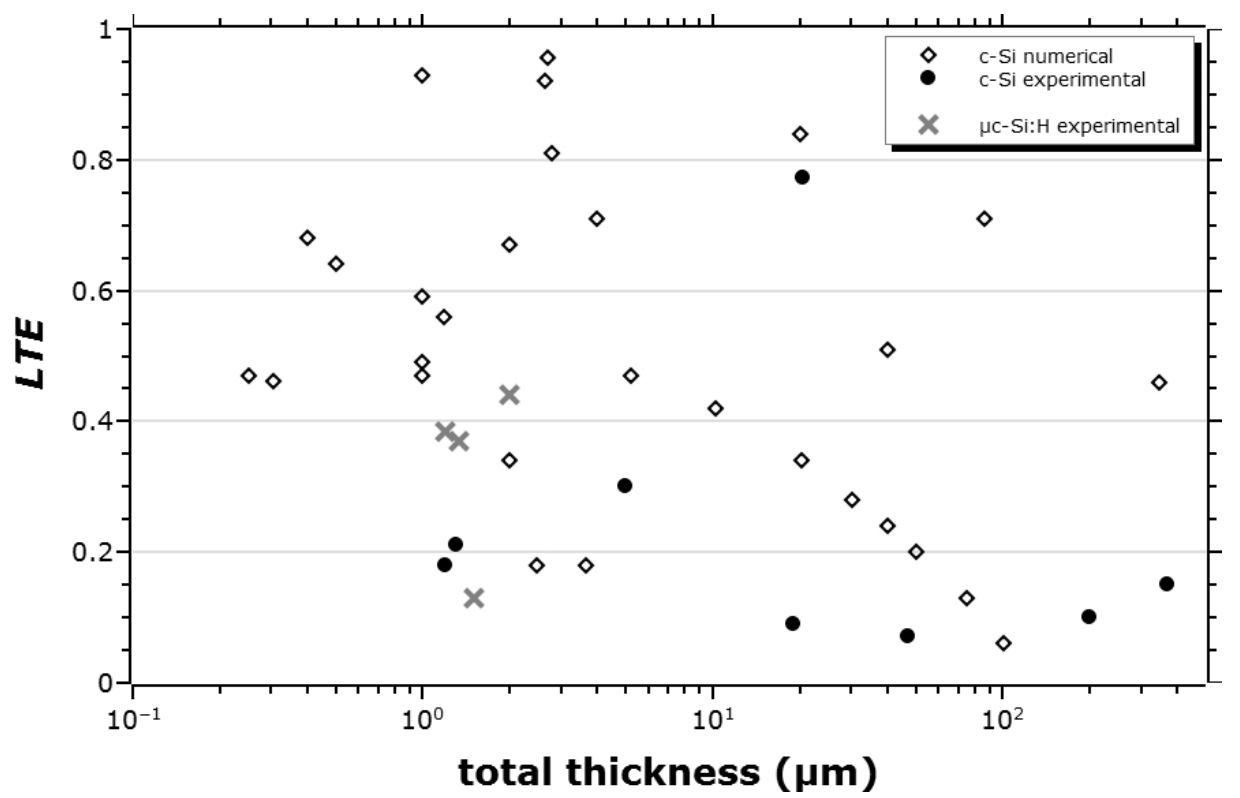

FIG. 2.17: The calculated light trapping efficiency (LTE) of light trapping structures realized or proposed in c-Si found in the literature (see Eq. 2.25). All $\mu \mathrm{c}-\mathrm{Si}: \mathrm{H}$ data points were assessed with the optical constants of c-Si [11]. While the $L T E$ is, in principle, independent of the absorber thickness, the highest performing structures operate in the $1-5 \mu \mathrm{m}$ range, which may relate to the fact that the benefit of light trapping is maximal in this thickness range. Notably, the solar cells with the highest efficiency (e.g. the $370 \mu \mathrm{m}$ thick PERL cell) are not necessarily the best light trapping structures, highlighting the difference between the LTE and the absolute efficiency. This difference also highlights the importance of anti-reflection coatings, as already shown in Fig. 2.11.

Figure 2.17 summarizes the outcome of the assessed structures, while the corresponding short-circuit currents are listed in Tab. 2.1 to Tab. 2.3. For the assessment of the structures found in the literature, I applied the following four methods.

1. Absorption spectra. When only the numerical absorption spectra were shown [85], I used the free software tool Plot Digitizer from sourceforge.net and the standard global solar spectrum AM1.5G [89] in order to calculate the $L T E$ of the structure.

2. Optical constants. As theoretical studies mainly use the optical constants provided by Green [11] or Palik [90], I tried to take this into account for the LTE. However, if $J_{r e f}$ appeared too close to the current of a single-pass traversal, I recalculated the reference with the same optical constants, e.g [91]. 
3. Effective thickness. When authors compared their proposal to reference samples with the effective thickness, e.g [59], I recalculated the short-circuit currents $J_{M B}$ and $J_{L L}$, since in Eq. 2.25 all currents are defined for the same (total) absorber thickness. For the planar reference $J_{r e f}$, I decided to use the ideal short-circuit current $J_{M B}$ in order to establish a minimum for the light trapping performance.

4. Parasitic absorption. When authors compute the electrical current from absorption spectra, the charge-collection is generally assumed to be $100 \%$, i.e. every photogenerated charge-carrier contributes to the short-circuit current. Since the total absorption is decomposed into useful absorption in the silicon and parasitic absorption in other layers, the electrical current must refer to the sole active absorption only. I therefore excluded studies where I suspected parasitic influences. My criterion was a too high absorption near the wavelength bandgap of silicon.

For a $300 \mu \mathrm{m}$ thick silicon wafer, the absorption at $1100 \mathrm{~nm}$ wavelength will be greater than $7 \%$ without back reflector and anti-reflection coating. For a $1 \mu \mathrm{m}$ thin silicon slab with $100 \%$ anti-reflection coating and lossless mirror, it requires a path length enhancement factor of $4 n^{2}$ to absorb almost $2 \%$ of the incident light, if $n=3.54$ refers to the refractive index at $1100 \mathrm{~nm}$ wavelength. However, if the metal mirror were $99 \%$ reflective, the parasitic loss may already sample to $1-0.99^{\left(2 n^{2}\right)}>20 \%$, assuming the light is trapped by total internal reflection at the front and the metal mirror at the back.

The review allows me to conclude that light trapping is now close to being a solved problem, because several studies were able to achieve an $L T E$ very close to the ideal 100\% performance. Naturally, demonstrating such high light-trapping efficiencies in real working solar cells still remains a challenging task.

As the key function of light-trapping structures is to enhance the absorption efficiency of a solar cell, it remains imperative to minimize the influence of the optical design on other process mechanisms that are involved in the conversion of the absorbed solar energy into electricity. So far, I could identify only one experimental structure approaching a $L T E \approx 80 \%$, where nanotextures at the front 
and random pyramids at the back were designed in respect to high performance in anti-reflection and in light-trapping, respectively. The design also uses a silver mirror as back reflector, after a $100 \mathrm{~nm} \mathrm{Si}_{2}$ was thermally grown on both sides of the structured $20 \mu \mathrm{m}$ thick silicon absorber [83].

\begin{tabular}{l|r|r|r|r|r|r} 
LTE & thickness $(\mu \mathrm{m})$ & $J_{\max }$ & $J_{\text {ref }}$ & $J_{L L}$ & $J_{M B}$ & reference \\
\hline \hline 0.96 & 2.7 & 33.9 & 23.2 & 36.2 & 25.0 & {$[75]$} \\
0.93 & 1.0 & 29.1 & 15.0 & 33.5 & 18.3 & {$[92]$} \\
0.92 & 2.7 & 35.7 & & 36.6 & 25.0 & {$[93]$} \\
0.84 & 20.0 & 37.3 & 31.3 & 41.1 & 34.0 & {$[91]$} \\
0.81 & 2.8 & 34.6 & & 36.8 & 25.3 & {$[76]$} \\
0.71 & 4.0 & 32.0 & 24.8 & 37.6 & 27.6 & {$[43]$} \\
0.71 & 86.7 & 42.0 & & 43.3 & 38.8 & {$[59]$} \\
0.68 & 0.4 & 21.8 & 10.3 & 29.6 & 12.5 & {$[94]$} \\
0.67 & 2.0 & 29.4 & 21.0 & 35.7 & 23.0 & {$[43]$} \\
0.64 & 0.5 & 21.5 & 12.0 & 28.7 & 13.8 & {$[43]$} \\
0.59 & 1.0 & 25.4 & 16.5 & 33.3 & 18.3 & {$[43]$} \\
0.56 & 1.2 & 27.6 & 19.4 & 34.1 & 19.4 & {$[95]$} \\
0.51 & 40.0 & 36.4 & 33.7 & 42.3 & 37.0 & {$[96]$} \\
0.47 & 5.2 & 33.6 & & 38.4 & 29.2 & {$[95]$} \\
0.47 & 0.3 & 16.4 & 8.3 & 27.2 & 9.9 & {$[43]$} \\
0.47 & 1.0 & 24.2 & 17.1 & 33.5 & 18.3 & {$[97]$} \\
0.46 & 0.3 & 20.1 & & 30.2 & 11.4 & {$[98]$} \\
0.46 & 346.7 & 43.0 & & 44.5 & 41.7 & {$[59]$} \\
0.42 & 10.2 & 35.7 & & 39.9 & 32.6 & {$[95]$} \\
0.34 & 2.0 & 23.6 & 19.3 & 35.8 & 23.1 & {$[85]$} \\
0.34 & 20.3 & 37.2 & & 41.2 & 35.2 & {$[95]$} \\
0.28 & 30.2 & 37.9 & & 41.9 & 36.3 & {$[95]$} \\
0.24 & 40.2 & 38.3 & & 42.3 & 37.1 & {$[95]$} \\
0.20 & 50.2 & 38.6 & & 42.7 & 37.6 & {$[95]$} \\
0.18 & 2.5 & 26.7 & & 36.5 & 24.5 & {$[99]$} \\
0.18 & 3.7 & 29.0 & & 37.5 & 27.1 & {$[100]$} \\
0.13 & 100.3 & 39.1 & & 43.2 & 38.5 & {$[95]$} \\
0.06 & & 39.4 & & 43.5 & 39.2 & {$[95]$}
\end{tabular}

TAB. 2.1: The $L T E$ of numerical structures using c-Si; only the best proposals in each reference were considered. The short-circuit currents are given in $\mathrm{mA} / \mathrm{cm}^{-2}$. 


\begin{tabular}{l|r|r|r|r|r||r} 
LTE & thickness $(\mu \mathrm{m})$ & $J_{\max }$ & $J_{\text {ref }}$ & $J_{L L}$ & $J_{M B}$ & reference \\
\hline \hline 0.77 & 20.5 & 39.3 & & 40.6 & 34.8 & {$[83]$} \\
0.30 & 5.0 & 17.5 & 14.7 & 38.3 & 29.0 & {$[101]$} \\
0.21 & 1.3 & 15.5 & 12.5 & 34.4 & 20.1 & {$[102]$} \\
0.19 & 1.5 & 15.4 & 12.8 & 34.8 & 20.9 & {$[103]$} \\
0.15 & 370.0 & 40.9 & 40.5 & 44.5 & 41.8 & {$[104]$} \\
0.10 & 200.0 & 41.0 & & 44.1 & 40.6 & {$[105]$} \\
0.09 & 19.0 & 35.5 & & 41.1 & 35.0 & {$[106]$} \\
0.07 & 47.0 & 37.8 & & 42.6 & 37.4 & {$[107]$}
\end{tabular}

TAB. 2.2: The LTE of experimental structures using c-Si; only the best proposals in each reference were considered. The short-circuit currents are given in $\mathrm{mA} / \mathrm{cm}^{-2}$.

\begin{tabular}{l|r|r|r|r|r||r}
$L T E$ & thickness $(\mu \mathrm{m})$ & $J_{\max }$ & $J_{\text {ref }}$ & $J_{L L}$ & $J_{M B}$ & reference \\
\hline \hline 0.44 & 2.0 & 28.7 & & 35.8 & 23.1 & {$[108]$} \\
0.38 & 1.2 & 20.8 & 15.3 & 34.4 & 20.1 & {$[30]$} \\
0.37 & 1.3 & 17.7 & 12.4 & 34.5 & 20.2 & {$[109]$} \\
0.13 & 1.5 & 9.9 & 8.1 & 34.9 & 21.1 & {$[101]$}
\end{tabular}

TAB. 2.3: The $L T E$ of experimental structures using $\mu \mathrm{c}-\mathrm{Si}$ and qualitatively assessed with c-Si [11]; only the best proposals in each reference were considered. The short-circuit currents are given in $\mathrm{mA} / \mathrm{cm}^{-2}$.

\subsection{Concluding remarks}

Analyzing the highest performing light-trapping structures [75, 76, 83, 91-93] allows to define three important design aspects: the control and modulation of the optical phase, the structuring of the absorber layer from both sides and the impact of surface textures on the electrical performance.

\subsubsection{Phase engineering}

The benefit of a certain amount of disorder in periodic light-trapping structures was repeatedly confirmed in the past $[43,91,110,111]$, but some practical guidelines were given by Han and Chen [93], which were based on arguments of group theory as described in the following. 
At first, the authors classify the waveguide modes into categories according to their symmetry properties under mirror reflection. Afterwards, they show that the mirror symmetries need to be broken to improve absorption, because the presence of mirror planes in a unit cell results in certain modes not coupling to incident light. The authors conclude that the symmetries can be broken either by destroying the periodic arrangements of the unit cell or by distorting the shape of the objects within the unit cell. They then argue that all modes will couple to light if the optical phase of any mode is not symmetric under mirror reflection. Following the outlined strategy, the authors indeed achieve an $L T E=0.92$ for $600 \mathrm{~nm}$ high skewed pyramids on a $2 \mu \mathrm{m}$ thick c-Si absorber layer. Please note, that their method differs from breaking the symmetry of macroscopic structures where phase information is absent.

The conclusion, that a lack of structural symmetry improves the optical absorption, is also in agreement with results reported by other groups and for other geometries:

1. Peters et al. [112] compare and verify the similar light trapping properties of optimized periodic and stochastic structures having similar geometrical features. The choice of their texture was based on a particular pattern that had already been used to fabricate efficient thin-film silicon solar cells.

2. By the subtle superposition of multiple binary gratings, Martins and coworkers $[92,113]$ choose to tune the appropriate level of disorder into the unit cell via its Fourier spectrum. Their concept allowed the designers to obtain an $L T E=0.93$ with a $190 \mathrm{~nm}$ etched QR code-like pattern on a $1 \mu \mathrm{m}$ thick c-Si absorber. The design uses a 100\% reflective mirror on the back and a $70 \mathrm{~nm}$ nitride coating on the top to reduce avoidable reflection losses.

3. Ferry et al. [114] systematically study the relationship between photocurrent and the spatial correlations of random or designed surfaces. They evaluate four general classes of nanopatterns in terms of their Fourier transforms, which were obtained from AFM images, and used this as a basis for the calculation of the corresponding power spectral densities. The authors highlight that the essential design rule for strong photocurrent enhancements rests on the capability of tailoring the spatial frequencies in the structure to high scattering powers in the required range of optical frequencies. 
Engineering light-trapping structures in terms of their impact on the optical phase thus can be understood as a general principle for enhancing the absorption of thin-films. Accordingly, whether a structure has a periodic or random morphology then tends to be less important than its local geometrical features, like the size and shape of the objects.

\subsubsection{Dual structuring}

The benefit of structuring the absorber layer from both sides increases the degrees of freedom available to the designer, as different functions can be combined. For example, Wang et al. [76] suggested to optimize the front and back surface of a silicon slab for anti-reflection and light-trapping, respectively, due to the significant difference in the structural requirements. Their proposed dual-grating design uses circular nanocones as the basic building elements for the grating geometry on both the front and the back surfaces. However, its realization could turn out to be complicated by the detached back reflector configuration.

Structuring the absorber layer from both sides has often been proposed by many various authors [75-77, 115-119]. In fact, some of the best light-trapping structures listed in Tab. 2.1 to Tab. 2.3 actually adhere to this idea.

\subsubsection{Electrical performance}

If an optically very good structure is electrically detrimental, or vice versa, the power conversion efficiency of a solar cell will be severely reduced.

1. From cross-sectional transmission electron microscopy (TEM) images Li et al. [120] show that the inability of conformal coverage tends to create pinholes in the cell, which are responsible for the electrical shunts. Using a textured substrate with a too high surface roughness for the deposition of the absorber material thus can result in short-circuiting of the entire device. 
2. Sai et al. [108] are able to correlate the origin of carrier collection losses to the non-uniform coverage around concaves on the substrate surface. From SEM and TEM images they conclude that the period of a honeycomb lattice sets a threshold value for the maximum absorber thickness, which can be deposited onto the light-trapping structure free of cracks. Using a textured substrate with periodic structures and a too small period thus can result in electrical defects.

3. Texturing the silicon surface after the material deposition generally tends to increase the surface recombination velocity of charge carriers. However, Oh et al. [17] also measure higher Auger recombination rates in case the doping profile of the device was changed by the presence of the texture.

Auger recombination is understood as the recombination of an electronhole pair that is giving up its energy to an electron in the conduction band (instead to a photon). Since the extra kinetic energy of the Auger electron is given off to the silicon lattice, such lattice vibrations will likely increase the absorber temperature. Consequently, Auger recombinations are understood as heat loss, because solar energy is not converted into electricity.

Recently, Isabella et al. [75] tried to optimize the optical characteristics of a complete solar cell stack configuration by minimizing not only the parasitic absorption losses, but also the electrical losses. Their proposed light-trapping scheme uses a high aspect ratio texture at the front side for anti-reflective purposes and low aspect ratio random pyramids at the back side for efficient long-wavelength light scattering. The proposed design could theoretically $(L T E=0.96$ [75]) and experimentally $(L T E=0.77[83])$ achieve the best light trapping efficiency. 


\section{Chapter 3}

\section{Fabrication and characterisation of diffractive nanostructures}

\subsection{Introduction}

This chapter focuses on the methodological aspects, outlining the realization and characterization of my samples. Section 3.2 describes the nanolithography techniques, such as electron beam and nano-imprint lithography, used for generation of the pattern. The details of the etching process are then given in section 3.3. In section 3.4, I analyze the required characteristics of the absorption measurement setup in terms of sensitivity, accuracy and overall performance.

\subsection{Nanolithography techniques}

Diffractive light trapping structures have dimensions on the order of the wavelength of light; such structures require the control afforded by lithographic techniques. In contrast, wet etching techniques that can generate rough surface textures are cheaper and easier to implement, but they do not afford this control. 
I mainly used electron beam lithography (EBL), because of its flexibility. Since the long exposure times and set-up procedures required for EBL are clearly not acceptable for larger scale production of solar cells, I also developed nanoimprint lithography as a low-cost, high-performance alternative (see appendix section A).

\subsubsection{Electron beam lithography}

Electron beam lithography (EBL) uses a beam of focussed electrons to create patterns in a resist material. As the kinetic energy of the electrons is in the $\mathrm{keV}$ range, the electron's de-Broglie wavelength is in the picometre range. The resulting spotsize is only of order $1 \mathrm{~nm}$, however, as it is difficult to focus an electron beam with a high numerical aperture, so relatively small numerical apertures are used. The resist layer changes its solubility following electron exposure and the material becomes selectively removable in a subsequent development step.

Typical feature sizes are no smaller than $10-20 \mathrm{~nm}$, however, as the incoming electron beam is scattered in the resist layer; more importantly, most of the electrons pass through the resist layer, and only lead to exposure once they have been backscattered from the substrate, as illustrated in Fig. 3.1a.

While e-beam lithography is very versatile and offers a fast turnaround time from design to pattern, it is a serial process that exposes one pixel after the other, hence is relatively slow. The handicap of this pixel by pixel exposure may be illustrated best in a comparison to standard photolithography:

Electron beam resist has a typical exposure dose of $150 \mu \mathrm{C} / \mathrm{cm}^{2}$, or ten electrons per surface atom $\left(10 \mathrm{e} / \mathrm{nm}^{2}\right)$. If the current is around $10 \mathrm{nA}$, the exposure of a $2 \mathrm{~cm}^{2}$ surface grating would last $1 / 2$ day. In contrast, the clearing dose of $150 \mathrm{~mJ} / \mathrm{cm}^{2}$ required by S1818 photoresist is achieved in 5 seconds by a UV-LED array of $30 \mathrm{~mW}$ optical power. Photolithography, in this particular example, is thus a factor of $10^{4}$ times faster than e-beam lithography. 


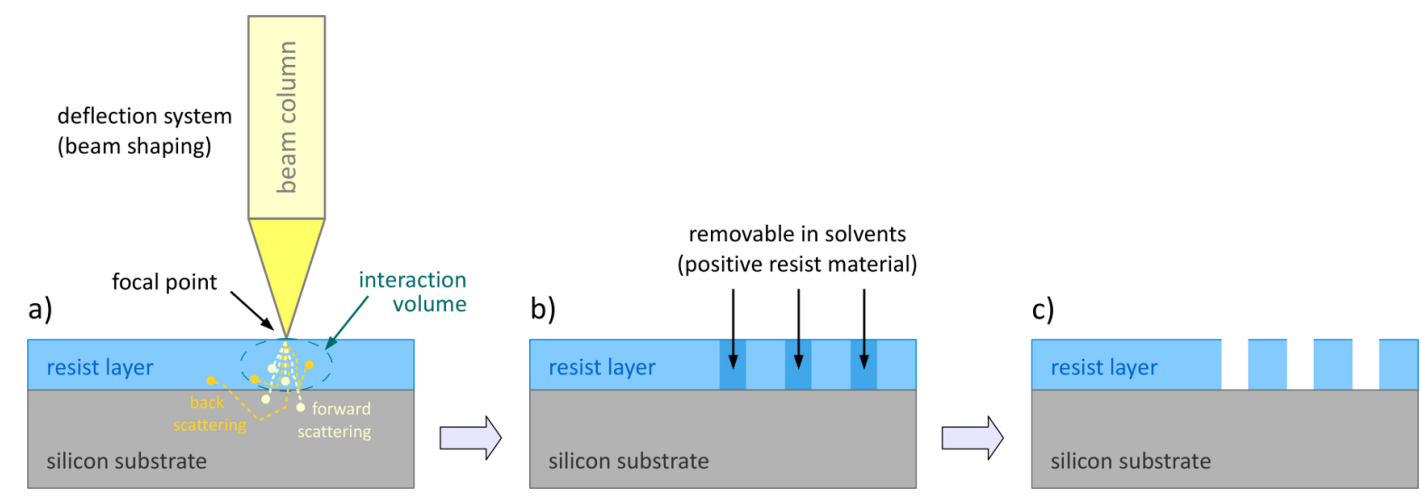

FIG. 3.1: From left to right: electrons are accelerated towards the specimen and expose the resist. The majority of the electrons do not stop in the resist but penetrate into the substrate. (a) Even if the beam diameter can be subnanometer in size at the focal point, the electrons may get scattered by the resist layer and get backscattered from the substrate, which effectively causes a widening of the exposure region. (b) Since the high-energetic electrons are able to break the molecular bounds, the exposed regions of a positive-tone resist become soluble in a developer. (c) After the developing step, the resist film is used as a mask to transfer the pattern into the substrate material by etching.

\subsubsection{The fabrication of diffractive structures}

Almost all my diffractive structures are realized on a silicon slab, using the positive-tone electron beam resist SX AR-P 6200 (CSAR62) from Allresist.

At first, a $250 \mathrm{~nm}$ thick layer of CSAR62 is spin-coated onto the cleaned and dried substrate at a rotation speed of $3000^{\mathrm{rpm}}$ for $60 \mathrm{sec}$, before the sample is left on a hotplate at $150{ }^{\circ} \mathrm{C}$ for $3 \mathrm{~min}$. The soft baking step removes any solvents and increases its adhesion to the substrate. The specimen is then immediately loaded into the vacuum chamber of the e-beam system (RAITH Voyager).

Once the working distance, focus, stigmation and beam distortion are adjusted, the structure is passed on to the pattern generator as a GDSII design, where it will finally be broken into a grid of exposure points. Since the Voyager is able to resolve geometrical objects with 18-bit precision, the smallest logically addressable element of a $500 \mu \mathrm{m}$ exposure field (or write-field) measures $\Delta x_{\text {min }}=500 \mu \mathrm{m} / 2^{18}=2 \mathrm{~nm}$ sidelength. In order to keep the exposure time on an affordable timescale, I typically use a pitch size of 4 or $8 \mathrm{~nm}$ without losses of spatial resolution. 
The dwell time $\Delta t$ of each pixel is determined by the current $I$, the step size $\Delta x$ and the area dose $D$, and is ultimately limited by the deflection speed of the pattern generator $(50 \mathrm{MHz})$ :

$$
\Delta t=D \cdot \Delta x^{2} / I \geq \Delta t_{\min } \equiv 20 \mathrm{~ns}
$$

If the design is patterned at a lower precision, e.g. by reducing the exposure points by $n$, the dwell time increases by a factor of $n^{2}$, allowing to select a larger beamcurrent by increasing the aperture. Since the beam diameter increases with aperture, high-resolution lithography can only be achieved with a small aperture and is relatively slow. Using the $40 \mu \mathrm{m}$ aperture, the beam current is around $0.6 \mathrm{nA}$. Using the maximum $500 \mu \mathrm{m}$ writefield of the Voyager, one such writefield typically takes between 5 and 10 minutes to pattern, depending on the complexity of the design. The quasi-random 2D supercell of section 4.3 measures almost $1 \mathrm{~cm}^{2}$ in size and is the largest pattern shown in this thesis, which, using these parameters, took almost 3 days to pattern. However, a new member in the photonics group was recently able to demonstrate $10 \mathrm{~h} / \mathrm{cm}^{2}$ by using the $100 \mu \mathrm{m}$ aperture in a high current mode.

At the end, the development step concludes the lithographic process; the sample is developed in X AR 600 from Allresist for 60 seconds and the development is quenched by rinsing in de-ionised water.

\subsubsection{Nanoimprint lithography}

Nanoimprint is an alternative lithography technique, which relies on the mechanical deformation of a polymer layer as a mold is pressed into it. The process is therefore analogous to the earliest form of writing, i.e. the imprint of pictographs into a wet clay tablet. This simplicity recommends nanoimprint lithography as a high throughput technique at low-cost. When the technique was invented in 1996 [63], Chou and his students were indeed inspired by the need for a low-cost and high resolution lithography method. 
Today, nanoimprint lithography has started to replace costly equipment tools in research laboratories. Yana et al. [121] and Sohn et al. [122], for example, achieved sub-50 nm resolution on a home-made setup. In the industrial sector, an exciting step was introduced by Molecular Imprints Inc., who used their innovative "Jet and Flash Imprint" technology - based on drop dispensing of UV curable resists for high resolution patterning - to become the market leader in patterning solutions: the new Imprio ${ }^{\mathrm{TM}} 450$ lithography system is capable of patterning entire $450 \mathrm{~mm}$ wafers at sub-20 nm resolution in less than 5 seconds, a technique that promises a low cost of ownership. Canon Inc. recently acquired this spin-out company of the University of Texas and intends to introduce nanoimprint lithography systems for volume production lines from 2015.

Despite these very promising developments, one must not forget that the masters are typically made by e-beam lithography, and that these masters only have a limited lifetime.

\subsubsection{The imprint step}

Nanoimprint relies on the plastic properties of a resist material, which needs to (inversely) replicate the nanostructured template as schematically shown in Fig. 3.2. The resist must therefore meet two important properties: a) the need for deformability requires the resist to have a lower Young's modulus than the stamp; b) the need to fill the trenches requires a low viscosity.

If a stamp is pressed into the resist layer, voids may form as the resist material is not able to fill the gaps in the stamp completely. In consequence, air bubbles may remain between the mold and the nanoresist. Applying pressure, temperature or a combination of both can reduce such voids, as long as the flowability of the nanoresist is increased (lower viscosity) by this curing step.

At best, the resist material comes as a liquid spin-on solution and is turned into a solid by a short curing process, with the stamp material assisting any capillary action. The problem of filling all the gaps was solved by the introduction of UV nanoimprint resist which is a low-viscosity solution that can be cross-linked by curing under UV light. 
(a)

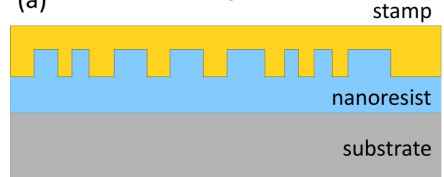

(b)

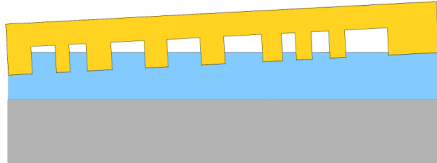

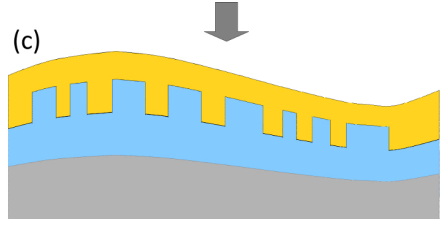

FIG. 3.2: If a stamp is directly pressed into a material that is softer than the stamp, the imprinted layer is mechanically deformed. By applying temperature, pressure, UV light or a combination of these, the (inverse) replica of the template is then produced in the cured material. However, the resist viscosity and deformability have decisive influence on the transferred pattern quality. Ideally, the cavities of the structured template will be completely filled (a), which requires the nanoresist to be of low viscosity. A high viscosity, or a non uniform surface contact between stamp and resist, can result in the formation of voids and in a loss of pattern fidelity (b). By reducing the Young's modulus of the stamp, a conformal contact can be made (c), but as the elastic contrast between stamp and nanoresist is reduced at the same time, a higher elasticity of the stamp material can also limit the pattern resolution. In addition, flexible stamps tend to get swollen by the nanoresist.

The downside of UV nanoimprint lithography, however, is that stamp materials need to be optically transparent. While the use of a transparent stamp may allow high-precision alignment, metallic stamps are often more suitable for large-scale production, because of their superior hardness and durability. In addition, if the nanoresist is to be used as an etch mask, UV-cross-linked nanoresists tend to be difficult to remove.

The three nanoimprint lithography techniques introduced above are compared in Tab. 3.1.

Direct nanoimprint requires a reliable control mechanism to precisely align stamp and substrate parallel to each other, often termed wedge error compensation, whereas thermal nanoimprint requires an accurate temperature control across the surfaces of substrate and stamp.

Considering these difficulties, I chose to develop a UV nanoimprint tool, because the technical requirements are less demanding: a UV light source for initializing the polymerization reactions, a quartz-disk for the stamp support and a weight for keeping the stamp profile pressed in the liquid resist solution at room temperature during the exposure. 


\begin{tabular}{|c|c|c|}
\hline direct nanoimprint & thermal nanoimprint & UV nanoimprint \\
\hline & nn & $\frac{14}{14}$ \\
\hline & n & \\
\hline direct embossing: & thermoplastic deformation: & radiation curing: \\
\hline press, step and repeat & heat, cool, step and repeat & flash, step and repeat \\
\hline $\begin{array}{l}\text { no or high viscous resist } \\
\approx 3 \mathrm{GPas}\end{array}$ & $\begin{array}{l}\text { spin-on resist solution } \\
\approx 3 \mathrm{Pas}\end{array}$ & $\begin{array}{l}\text { spin-on resist solution } \\
\approx 3 \mathrm{mPa} \mathrm{s}\end{array}$ \\
\hline $\begin{array}{l}\text { high pressures } \\
>1,000 \mathrm{~kg} / \mathrm{cm}^{2}\end{array}$ & $\begin{array}{l}\text { heating/cooling cycle } \\
\approx 150^{\circ} \mathrm{C}\end{array}$ & $\begin{array}{l}\text { UV light exposure } \\
\approx 1000 \mathrm{~mJ} / \mathrm{cm}^{2}\end{array}$ \\
\hline $\begin{array}{l}\text { strong need for uniform } \\
\text { surface contact }\end{array}$ & $\begin{array}{l}\text { requires thermal } \\
\text { matching between } \\
\text { substrate and stamp }\end{array}$ & $\begin{array}{l}\text { sets high demands on } \\
\text { chemical and physical } \\
\text { resist properties }\end{array}$ \\
\hline
\end{tabular}

TAB. 3.1: From left to right: direct nanoimprinting does not require any additional steps and can be used to make complex patterns via multiple imprints. However, this technique suffers from pattern relaxation issues and sets high demands on the uniformity of the surface contact due to the very high pressures used. Thermal nanoimprint lithography requires lower pressure by using elevated temperature for curing (central figure), but a thermal gradient between the substrate and the stamp can lead to pattern distortions during the cooling cycle, hence can affect the pattern fidelity. UV nanoimprint permits a high throughput via step and flash imprint lithography (right figure), because it does not require high pressures $\left(<1 \mathrm{~kg} / \mathrm{cm}^{2}\right)$ or a temperature control system; its capability, however, strongly depends on the chemistry of the photocurable resist material and it requires either a transparent substrate or a transparent stamp.

\subsubsection{The demoulding step}

Although nanoimprint appears to be a simple technique, several points need to be considered:

1. Stamp separation. If the nanoresist layer sticks to the stamp material, it can delaminate from the substrate at the demolding step, or both the imprinted replica and the template patterns could get damaged. 
2. Stress. Internal stress in polymers is a consequence of volume shrinkage (typically 5-15\%), for example, during solidification as a result of phase transformation or solvent evaporation. Stress can lead to surface instabilities, wrinkle formations, pattern relaxation/collapse and hence to a loss of replication fidelity.

3. Etch resistance. If the imprinted patterns need to be transferred from the resist into the substrate, the polymerized nanoresist must also function as a masking layer. Therefore, a good etch selectivity is mandatory for maintaining the pattern definition.

UV nanoimprint lithography succeeded in overcoming the above mentioned issues on a research and industrial level (Molecular Imprints Inc.):

(1) For a nondestructive release, the desirable requirements are a low interfacial tension and good structural stability of the imprinted patterns. Treating the stamp with a fluorinated silane therefore greatly helps to reduce any adhesive forces during the demoulding step [123]. In addition, the application of an oxygen plasma or a primer layer to the substrate - prior the deposition of the nanoresist - increases the binding properties and effectively addresses the peel-off issue.

(2) The internal stress is often reduced by the so called "soft-start" cure. A combination of initial slow polymerization (at low UV intensity) and then fast polymerization (at high UV intensity) allows the formation of tightly crosslinked microgels throughout the entire liquid resist volume (gelation), before the final vitrification process starts [124]. Otherwise, increasing the molecular weight of the polymer may also enhance its stress resistance (longer chains have a higher tensile strength), but increase its viscosity.

Finally (3), Peroz et al. [125] recently demonstrated a high etching selectivity of 1:15 between imprinted resist and silicon. 


\subsection{Pattern transfer by reactive ion etching (RIE)}

Having generated a resist pattern either by e-beam lithography or by nanoimprint, etching techniques are used to transfer the pattern into the desired material, with the resist acting as a mask. I can classify etching techniques into wet and dry etching.

a) Wet etching. Chemical liquids are used to remove material from the sample. If a specific pattern is defined by a resist mask on the sample, the material not protected by the mask will be etched away by the chemical solution. However, for substrate materials with a crystal structure, like silicon, the chemical reactions tend to occur along definite crystalline planes. Consequently, chemical solutions can not be used for transferring small sub-micrometer structures from a thin resist layer 1:1 into the surface of silicon.

b) Dry etching. The generation of ions and free radicals in a radio frequency (RF) plasma allows to remove the exposed areas of silicon, while a resist mask is protecting the remaining areas from either the physical bombardment by ions or the chemical etching by radicals. Reactive ion assisted etching processes (RIE) therefore tend to be highly directional, which enables me to transfer a pattern from an overlying masking material to the etched silicon surface with high fidelity.

Figure 3.3 schematically compares the wet and dry etching methods. Since the fabrication of sub-micron features requires a good profile control with vertical sidewalls in the silicon substrate layer, all nanostructures in my thesis are realized by means of RIE. 


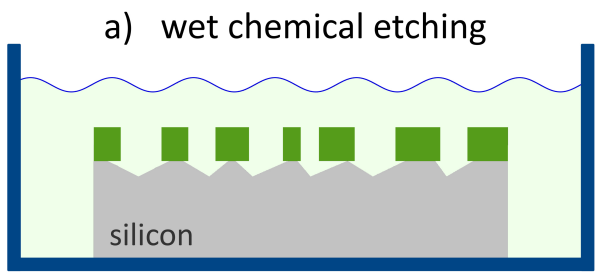

b) plasma etching

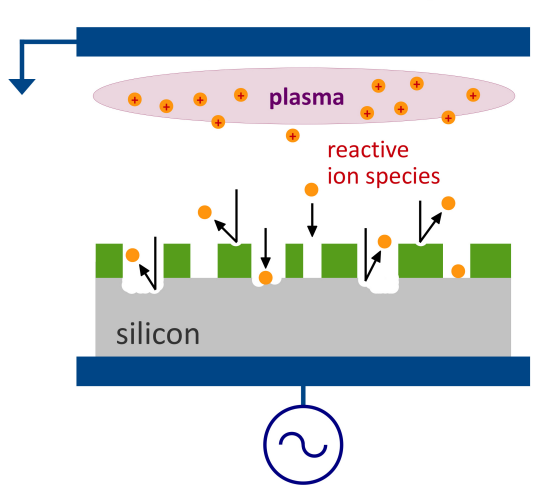

FIG. 3.3: Comparison of different etching techniques. The patterned resist (green) acts as a mask in both cases. I consider etching into silicon. a) wet chemical etching is the immersion of the silicon sample in a chemical bath of $\mathrm{TMAH}$ or $\mathrm{KOH}$. The material removal occurs along the crystal planes, which can result in an enlargement of the desired features due to undercuts of the masking resist material. b) dry etching done in vacuum, using a parallel-plate electrode configuration. An RF-frequency is applied to the electrode plates, thereby accelerating reactive radicals from the plasma towards the silicon sample. Reactive ion etching (RIE) is a combination of physical sputtering (which is anisotropic) and chemically driven reactions of activated neutrals (which is isotropic) on the material surface.

\subsubsection{Dry-etching in the RIE-system}

The removal of material requires the formation of volatile compounds by a combination of physical bombardment and chemical driven reactions. For example, the main purpose of etching silicon with a fluorine gas is the formation of volatile $\mathrm{SiF}_{4}$ molecules. However, while the physical sputtering is highly directional, the chemical reactions can cause undercuts and reduce the fidelity of the pattern transfer. In order to ensure all reaction products are volatile, it needs the right combination between the physical and chemical etching process.

One way to avoid undercuts is to protect the sidewalls with a polymer layer that can be formed during the plasma process. For example, the supply of chlorine or fluorocarbons facilitates the formation of $\mathrm{C}_{x} \mathrm{~F}_{y}$ film preferentially at the trench sidewalls, because the sidewalls are not directly exposed to the ion bombardment. A small hydrogen concentration has been shown to further assist the buildup of this passivation layer [126]. 
Therefore, I decided to use a $\mathrm{CHF}_{3}$ gas for the silicon etching and a graphite stage as the bottom electrode for increasing the generation of fluorocarbons.

The silicon etching thus becomes controllable by the fluorine concentration: the higher the fluorine concentration, the greater the ion bombardment and the more frequently chemical reactions occur, and, consequently, the more silicon is etched. For obtaining a high etch rate with smooth and near-vertical sidewalls, I fine tuned the parameter of the RIE process by the addition of fluorine $\left(\mathrm{SF}_{6}\right)$. As background contamination can result in a loss of process control, the etching takes place in an high vacuum environment ( $<30$ mTorr). The silicon etching recipe was optimized by all group members and is listed in Tab. 3.2.

After each etching step, I effectively remove any resist residuals by a 2 min oxygen plasma treatment, a warm 1165 solution around $30^{\circ} \mathrm{C}$ and a 15 min piranha bath $\left(\mathrm{H}_{2} \mathrm{SO}_{4}: \mathrm{H}_{2} \mathrm{O}_{2}=3: 1\right)$.

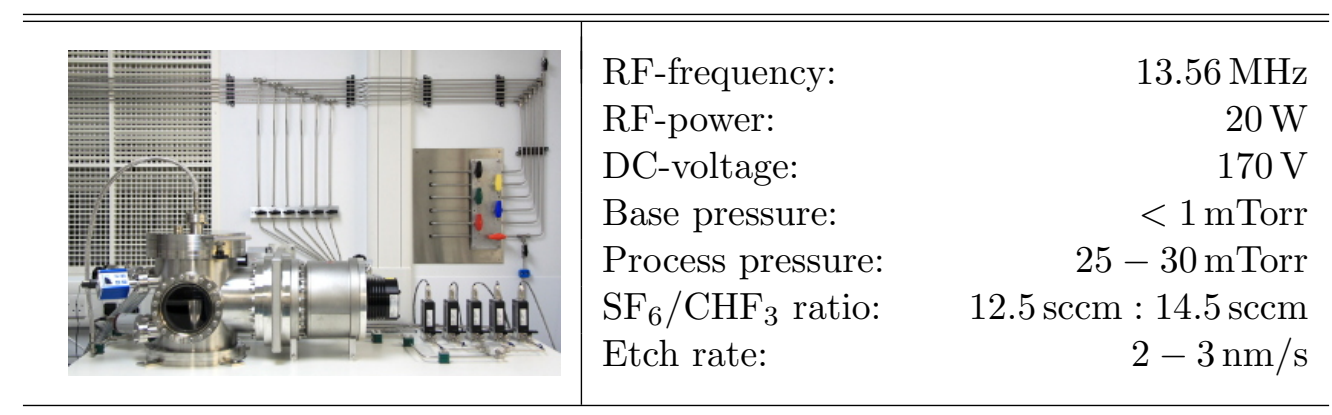

TAB. 3.2: The RIE system at York is a capacitively coupled RF plasma and set up for smooth sidewall etching of silicon on insulator (SOI). The graphite stage is the powered and smaller electrode plate, restricting the maximum sample size to $7 \mathrm{~cm}$ in diameter. The etch rate varies from $2 \mathrm{~nm} / \mathrm{s}$ (SOI) to $3 \mathrm{~nm} / \mathrm{s}$ (a-Si:H), allowing reasonable short process windows. 


\subsection{Absorption measurements}

In order to characterise my samples, I built a bespoke absorption measurement setup. Appendix section B provides detailed background information about this setup, while this section describes the calibration procedure.

For accurate absorption measurements, the total reflection $R$ and total transmission $T$ of a sample should be determined at the same time. However, standard spectrophotometers only allow to measure $R$ and $T$ one after the other and by repositioning the sample. When the spectra are obtained in sequence, the measurements could differ either in the angle of incidence or in the illuminated area of the sample, and therefore resulting in artefacts and inaccurate data sets, e.g. the absorption exceeds 1 or falls below 0 . In order to avoid such artefacts, I modified a standard integrating sphere such that $R$ and $T$ are detected simultaneously (see appendix section B).

The spectral absorptance $A(\lambda)$ is defined as the fraction of radiation absorbed at a specific wavelength $\lambda$, i.e. it is the ratio between the absorbed and the incident optical power. Calibration can be understood as the characterization of the experimental conditions, i.e. as a baseline scan. Each component in the setup influences the detector signal. For example, a sample mounted in the centre of the integrating sphere introduces a systematic error in the measurement, described in Fig. B.1 (see appendix). As long as all subsequent measurements remain affected in the same way, any signal changes can be directly contributed to the absorption of the specimen. Therefore, the more accurate I can make the baseline-scan, the smaller the measurement error will be.

I performed the calibration after a $60 \mathrm{~min}$ warm up phase of all instruments, which reduces thermal fluctuations and allows a precise absorption measurement $A(\lambda)$, that can be compared to a computer simulation later. 


\subsubsection{Reference measurement - thin films}

Thin-films of high-index materials are good references for assessing the measurement setup, because the thin film acts as a Fabry-Perot cavity, and its properties can be easily calculated via the well-known Airy function.

I used two reference samples; the first is a $1 \mu \mathrm{m}$ thin-film of crystalline silicon on glass (Fig. 3.4), the second is a polished $220 \mathrm{~nm}$ thin-film of crystalline silicon on a $2 \mu \mathrm{m}$ thick insulating oxide layer (SOI), which has been thermally grown on a standard silicon substrate wafer before (Fig. 3.4).

Even though the measurement and simulation match reasonable well, the comparison suggests an insufficiently collimated laser beam, because the measured Fabry-Perot fringes show a reduced dynamic range.

In order to produce a more collimated beam, a doublet lens would need to be placed at its focal distance from the monochromator. Using an achromatic doublet lens at the exit port of the monochromator not only can help to achieve a tighter focus but also a superior off-axis performance compared to the planoconvex lens used in the setup.

However, a non perfectly collimated laser beam will not severely affect the characterization of diffractive structures, because roughness or surface patterns scatter a large part of the incident light off-axis. 


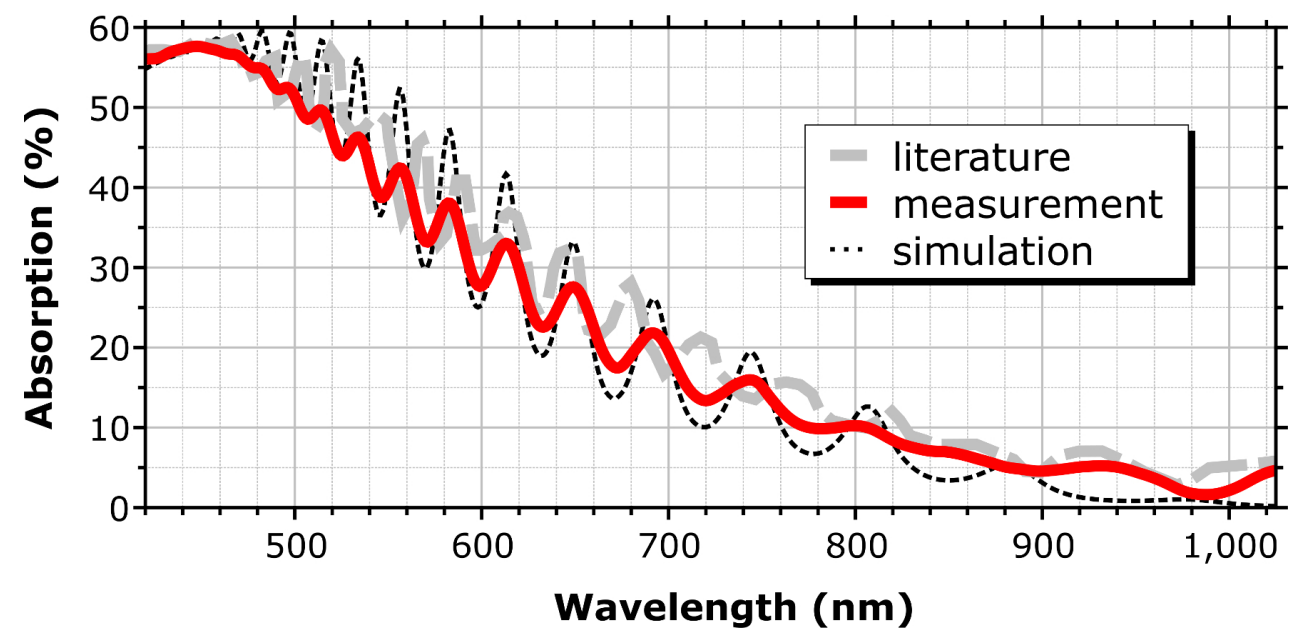

FIG. 3.4: Comparison of thin-film absorption of a $1 \mu \mathrm{m}$ thin-film of crystalline silicon on glass. The red solid line refers to the measured absorption, the grey solid line shows the absorption measurement of a similar sample found in the literature [127] and the black dotted line represents the theoretical Airy curve. The average values agree extremely well, highlighting that the setup is calibrated well. The small deviation in the magnitude of oscillation suggests an incoherent collimated input beam; the Airy curve assumes a plane wave input, while the measurement uses a collimated point source.

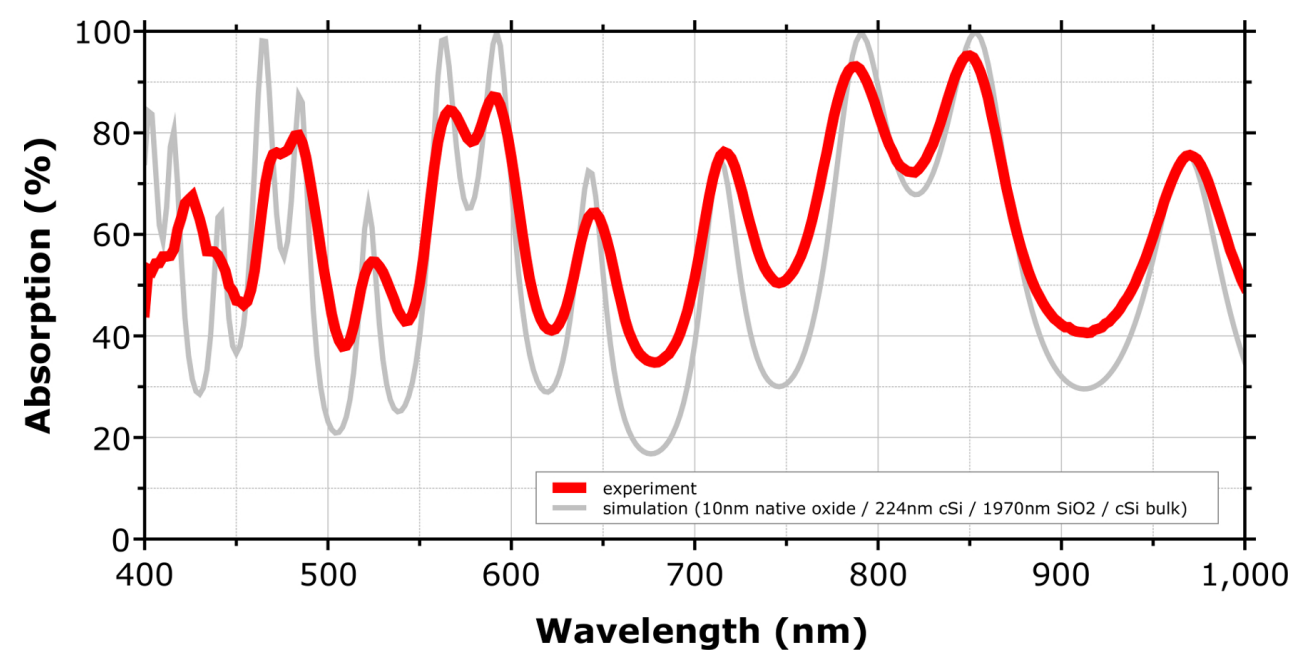

Fig. 3.5: Comparison of thin-film absorption of a $220 \mathrm{~nm}$ thin-film of crystalline silicon on a $2 \mu \mathrm{m}$ thick oxide layer of a standard SOI wafer. The red solid line refers to the measured absorption, the grey solid line shows the simulated absorption spectrum. The reduced dynamic range of the measured Fabry-Perot fringes compared to the model is probably due to a non perfectly collimated or partly coherent beam as mentioned above. 


\subsection{Summary}

This chapter outlines the fabrication process used for the nanostructures in my thesis.

I first discussed the key aspects of electron beam lithography, because the e-beam system at York (RAITH Voyager) offered me a fast turnaround time from design to pattern. Electron beam lithography, however, is a serial process that exposes one pixel after the other, so it is still a non acceptable lithography technique in larger scale productions of solar cells. Therefore, I also explore the technical possibilities offered by nanoimprint lithography.

After weighing the three most common nanoimprint methods against each other, I chose to design a UV nanoimprint tool based on LEDs, as it does not require high pressures or a temperature control system. Using a bi-layer hardPDMS/PDMS stamp in combination with the nanoresist AMONIL, the imprint process was successfully tested on a tool constructed by myself (described in appendix section $\mathrm{A}$ ).

The lithography process is followed by a RIE dry-etching, which allows me to transfer the fabricated structures from the resist material into the silicon substrate without loss of fidelity. The need for smooth and near-vertical sidewalls involved an RIE parameter study.

Finally, I outlined how the absorption of structured samples can experimentally be assessed on a home-made characterization set-up. Therefore, I had modified a standard integrating sphere such that the total reflection and transmission are detected simultaneously (see appendix section B). The measurement of crystalline silicon thin-films on glass show a good agreement with numerical calculations. 


\section{Chapter 4}

\section{Achievements}

\subsection{Introduction}

This chapter outlines two key experiments performed in my $\mathrm{PhD}$ project:

1. The first experiment highlights a simple layer transfer technique. In a proof-of-principle, I demonstrate the ability to pattern a silicon thin-film independently from both sides in section 4.2. My technique could support the study of more efficient light trapping designs without being constraint by design parameters.

2. The second experiment, outlined in section 4.3, concentrates on a fair assessment of two different light-trapping mechanisms, i.e. diffractive vs plasmonic. This comparison is jointly undertaken with colleagues from the University of Catania and it highlights the importance of avoiding any optical loss mechanisms in novel light-trapping designs. 


\subsection{Dual gratings by a simple layer transfer tech- nique}

The research community initially focused on one-dimensional (1D) gratings to study the key requirements of light-trapping, because they are less complex and therefore potentially easier to fabricate than two-dimensional (2D) gratings. Although 1D designs are simpler to analyze, e.g. numerical simulations require less computational power, 1D structures have a tendency to be most effective for only one polarization.

2D designs are more complex than 1D gratings but show two advantages. Firstly, they can allow to address both polarization states. Secondly, they provide access to a significantly larger number of diffraction orders. For these two reasons higher absorption enhancements are expected from 2D gratings and were widely proposed to further explore the interaction of light with textured surfaces.

A logical step and further extension was to use gratings on both sides of the film, because structuring the absorber layer from both sides increases the degrees of freedom available to the designer, at the cost of increased complexity though. Since 1D gratings tend to have a higher diffraction efficiency for one polarization, using two different 1D gratings on each side could be advantageous for targeting both polarization states separate from each other. In addition, the wavelength spectrum at the rear side is significantly reduced compared to the front side, because the silicon absorber functions as a long-pass filter. A back-side lighttrapping structure consequently needs to be designed only for wavelengths that are not absorbed in their first pass through the silicon slab.

Dual structures were studied by the community $[59,76,118,119]$, but theoretical proposals were generally limited to supercell-type calculations (where the period on one side is the multiple of that on the other) and experimental structures were often restricted to those with the same period on both sides, i.e. deposited by conformal growth techniques (where the structural features of the substrate go all the way through the absorbing material).

Developing a simple layer transfer technique would therefore allow to assess experimentally the light-trapping performance of any kind of structure without numerical nor technological constraints. 


\subsubsection{Why dual gratings ?}

Although diffraction is a wavelength-dependent phenomenon, light-trapping structures need to operate broadband in order to exploit the incident sunlight. The ability to independently structure both sides of a thin-film absorber could allow one to combine two different optical functions then: while the top grating could be optimized for light in-coupling and anti-reflection action in the visible part of the spectrum, the longer wavelengths could be diffracted by the bottom grating design so as to enhance their optical path length through the absorber slab. For example, light below $600 \mathrm{~nm}$ wavelength is absorbed in silicon within the first micrometre, whereas light above $800 \mathrm{~nm}$ wavelength usually requires path lengths of at least $10 \mu \mathrm{m}$. Using a dual grating design would thus allow one to take advantage of the full benefits that a texture can offer and may even allow thin-film cells to approach the Lambertian limit more closely.

Thin-film absorber layers, however, are generally obtained by vacuum deposition methods onto a bulk substrate. The substrate then impedes direct access to the back side of the absorber slab, which is therefore not available for patterning. In the following, I will briefly list a few currently known layer transfer methods, before outlining a novel but simple alternative technique. As a proof-of-principle demonstration and by choosing two different designs on each side of a silicon slab, I was able to fabricate a dual grating structure and managed to show good agreement between the measured and the simulated absorption spectra.

\subsubsection{A novel layer-transfer technique}

Zeng et al. [128] were able to directly bond the silicon device layer of a SOI wafer to a new handle wafer and to remove the original substrate by etching afterwards. The bonding of the silicon surface to the new substrate, however, was very unreliable and involved a high temperature annealing step between 600 and $1000^{\circ} \mathrm{C}$. 
Cruz-Campa et al. [129] were able to demonstrate how $14 \mu \mathrm{m}$ thin and $15 \%$ efficient silicon solar cells can be released from the original substrate wafer. However, the processing involved five etching steps and long submersion in potassium hydroxide $(\mathrm{KOH})$.

Petermann et al. [130] produced a mesoporous double layer by electrochemical etching, which facilitated the lift-off of a vapour deposited silicon layer. They then used this technique to fabricate a $43 \mu \mathrm{m}$ thick and $19 \%$ efficient silicon solar cell.

All the three techniques, mentioned above, require expensive vaccuum equipment tools and are not easy to reproduce. Instead, a simple layer transfer method could be characterised by

1. low-cost (avoids the need for vacuum equipment),

2. easy handling and processing steps,

3. unaffected silicon material properties,

4. short waiting times (avoids etching or high temperature cycles),

5. high yield.

My novel idea combines the use of an optical adhesive with the transfer of the thin-film to a new carrier. This combination automatically meets the five criteria mentioned above. For a microscope glass slide as the carrier, a high yield may also be obtained, because optical adhesives naturally conform to glass.

Since the patterning of a material generally involves at least one lithography step, the glue must retain its optical and adhesive properties after short baking periods or chemical solutions that are typically used to develop/strip a resist material.

The optical adhesive NOA61 from Norland fulfils these secondary conditions. According to the manufacturer, NOA61 withstands temperatures up to $260^{\circ} \mathrm{C}$ as a coating on the surface of a substrate for three hours. I tested the glue to be highly resistant to most chemicals (even to $\mathrm{HF}$ ) and temperatures up to $180^{\circ} \mathrm{C}$ on a hot plate for short times. 
To guarantee a strong bonding to NOA61, the surfaces of both the foreign substrate and the thin-film layer must be clean, dry and highly hydrophilic, which can generally be achieved by piranha solutions or a plasma ashing with oxygen ions.

Before the optical glue is then drop casted onto the thin-film, the surface energy can even further be increased by pre-heating the samples on a hot plate above $100{ }^{\circ} \mathrm{C}$. The heat treatment of NOA61 improves the homogeneity of the adhesive and also avoids the formation of cracks, while a spinning process afterwards can help to achieve a flat and uniform coating layer.

At the end, the microscope glass slide is simply placed onto the liquid glue as the new carrier and cured under UV light $\left(3 \mathrm{~J} / \mathrm{cm}^{2}\right)$. Following exposure, the two carriers can be simply prised apart, which finishes the layer transfer process. The technique relies on the fact that the thin film adheres more strongly to the cured NOA61 than to the original carrier.

I was able to transfer amorphous silicon thin-films up to $4 \mathrm{~cm}^{2}$ surface area by using the above procedure.

\subsubsection{Proof of principle demonstration}

As a proof of principle, I chose to pattern a $400 \mathrm{~nm}$ thin-film of hydrogenated amorphous silicon (a-Si:H) from both sides. The process flow is schematically shown in Fig. 4.1.

\subsubsection{Fabrication and design parameters of the dual-grating}

As 2D square lattices of holes have previously been considered for light trapping [43], I decided to use two such lattices of the same period of $300 \mathrm{~nm}$, but which greatly differ in their fill factors. Keeping the period the same on both sides of the absorber makes the subsequent numerical comparison easier. 
a)

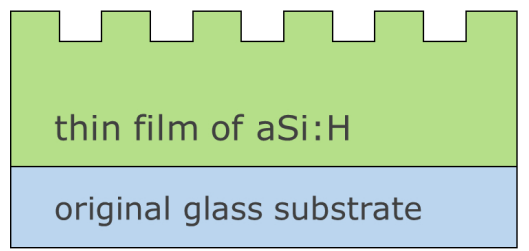

d)

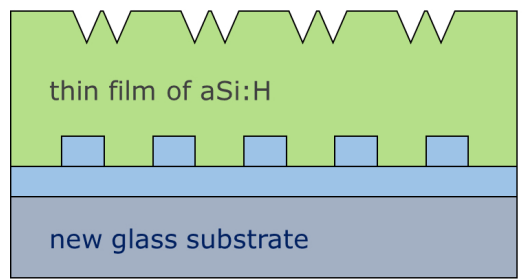

b)

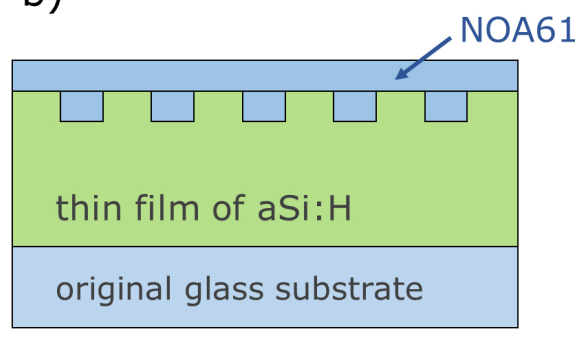

C)

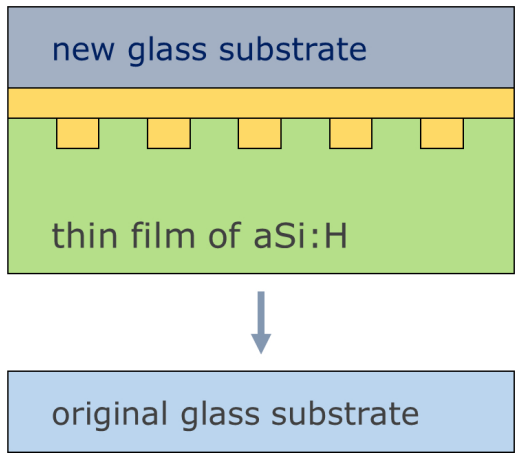

FIG. 4.1: The layer transfer technique relies on the fact that the thin-film adheres more strongly to the cured optical adhesive NOA61 than to the original carrier. (a) Following the deposition and patterning of the thin-film, (b) the optical adhesive NOA61 from Norland is spin coated onto the surface. (c) A glass slide is then placed onto the liquid glue as the new carrier. NOA61 will solidify under UV light exposure $\left(3 \mathrm{~J} / \mathrm{cm}^{2}\right)$, which permits the two carriers to be prised apart, thereby leaving the thin-film bonded to the new carrier. (d) Since the layer-transfer technique allows us to get access to the back side of the thin-film, both sides of the film can now be structured independently and without any restrictions on the design parameters.

The fabricated top and bottom gratings can be seen in Fig. 4.2 and are clearly distinguishable by their design aspects. The bottom grating appears as a welldefined 2D lattice of holes, while the topography of the top grating rather resembles a square lattice of star-like columns in air. Both structures were realized by electron beam lithography and reactive ion etching (see chapter 3). However, since warm chemical solutions (above $30^{\circ} \mathrm{C}$ ) can dissolve NOA61, the resist material was effectively removed by means of an oxygen plasma, instead of using the standard resist remover 1165.

A top view image of the final sample is shown in Fig. 4.3 along with a crosssection of the completed structure. 


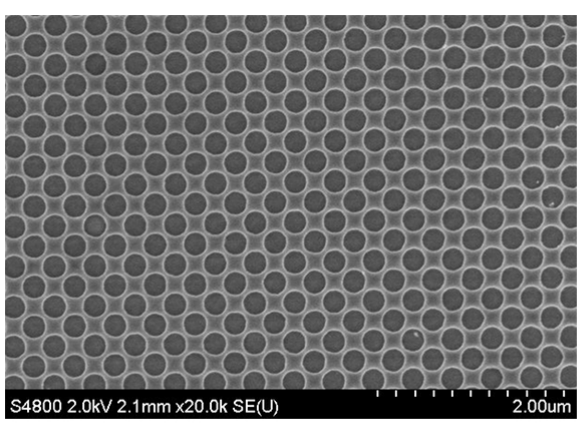

(a)

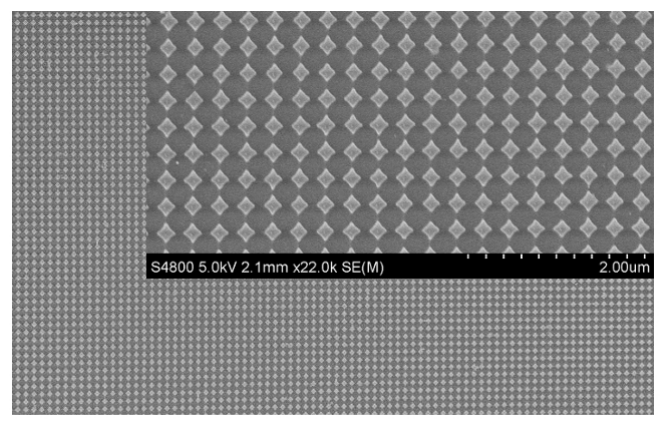

(b)

FIG. 4.2: Scanning electron microscopy (SEM) images of the 2D square lattices used for the dual-grating approach. Both gratings have the same period of $300 \mathrm{~nm}$ in order to facilitate the computational comparison. (a) The bottom grating is a photonic lattice of holes in a-Si:H with $240 \mathrm{~nm}$ diameters and $60 \mathrm{~nm}$ etching depth. Norland's optical adhesive NOA61 has highly transparent properties; its refractive index of 1.56 is very similar to $\mathrm{SiO}_{2}$. (b) The holes of the top grating touch each other, as the diameter was set equal to the lattice period. Therefore, the structure rather simulates star-like columns of a-Si:H in air. The nominal etch depth is $80 \mathrm{~nm}$.

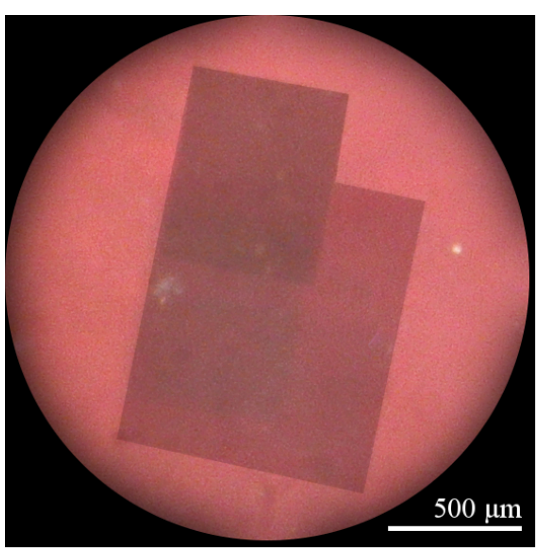

(a)

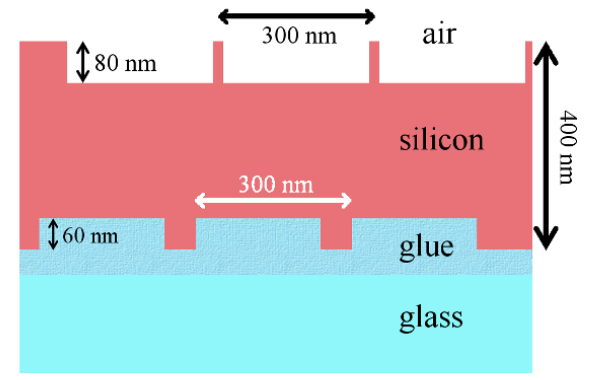

(b)

FIG. 4.3: (a) Microscope image (top view) of the 2D dual interface grating fabricated in a $400 \mathrm{~nm}$ thin a-Si:H slab on glass. The top and bottom grating are independently patterned by electron beam lithography and have been offset to allow individual optical characterisation. (b) A cross-sectional scheme of the fabricated final structure. The glue has the same optical properties as the glass substrate. 


\subsubsection{Measurements and results}

First, the measured absorption spectrum of the as-deposited a-Si:H film is compared with a numerical simulation in Fig. 4.4. The comparison reveals a good agreement and also verifies the optical material properties used in the model. The required dielectric function was obtained by variable angle spectroscopic ellipsometry (VASE) conducted by M. Patrini from the University of Pavia.

The absorption measurements of the bottom, top and double-sided gratings are then compared in Fig. 4.5 to the spectra calculated by P. Kowalczewski of the University of Pavia. In all cases, a very good agreement is seen. Notably, the spectrum of the dual-grating structure combines features of both individual gratings.

Tab. 4.1 summarizes the measured integrated absorption and the absorption enhancement achieved by the different structures. Since the optical properties of NOA61 are designed to resemble those of glass, the refractive index of the glue and the glass substrate are kept the same in all calculations.

Since the top grating provides anti-reflection action and diffraction into the aSi:H slab, it outperforms the bottom grating ( $32 \%$ vs $25 \%$ ). For example, most of the incident short-wavelength light will be absorbed during its first pass through the material layer, such that the bottom grating alone can efficiently address only the (non-reflected) longer wavelengths.

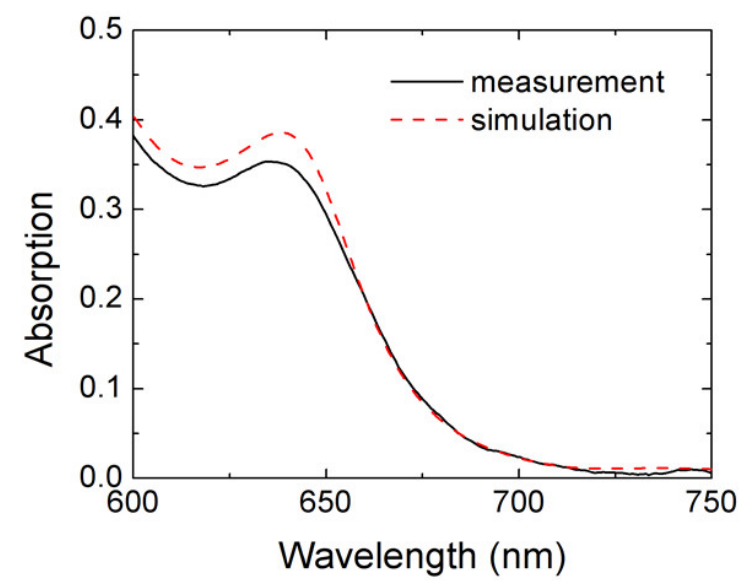

FIG. 4.4: Comparison of the measured (solid line) and numerical simulated (dashed line) absorption spectra of the unpatterned $400 \mathrm{~nm}$ thin a-Si:H film deposited by plasma enhanced chemical vapor deposition (PECVD). 


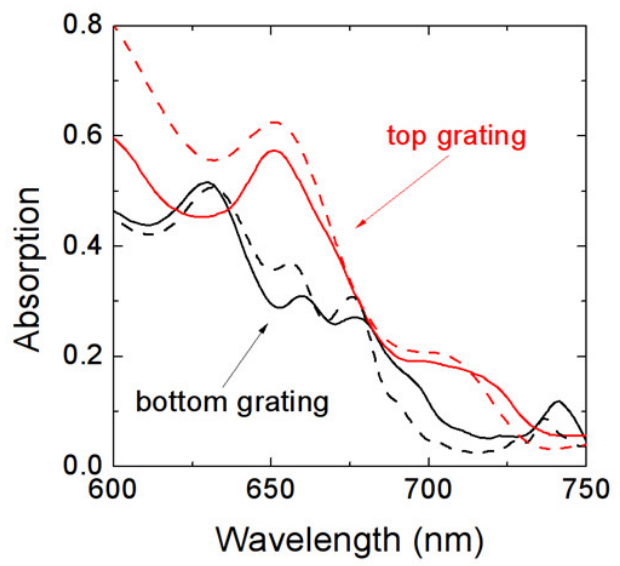

(a)

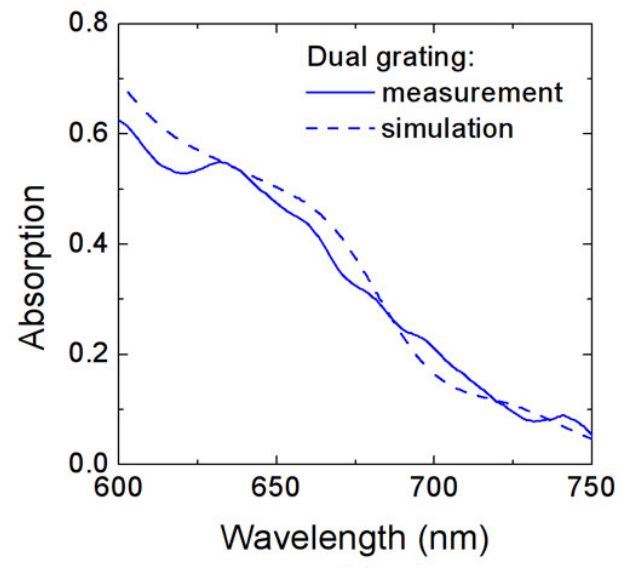

(b)

FIG. 4.5: Comparison of the measured (solid line) and numerical simulated (dashed lined) absorption spectra of the top or bottom patterned $400 \mathrm{~nm}$ thin a-Si:H film (a) and the dual-grating structure (b). A very good agreement is found, except for the top-grating of Fig. 4.3 in the short-wavelength range.

\begin{tabular}{llllll}
\hline & unpatterned & bottom & top & bottom+top & unpatterned-ARC-Ag* \\
\hline absorption & $15 \%$ & $25 \%$ & $32 \%$ & $33 \%$ & $34 \%$ \\
enhancement & $0 \%$ & $65 \%$ & $110 \%$ & $115 \%$ & $110 \%$ \\
\hline
\end{tabular}

*unpatterned film with ARC and silver BR (simulated structure for comparison)

TAB. 4.1: The integrated absorption of a $400 \mathrm{~nm}$ thin a-Si:H slab on glass with or without pattern are listed for the wavelength range from $600 \mathrm{~nm}$ to $750 \mathrm{~nm}$. The results are also compared with a modelled reference sample consisting of a $\mathrm{Ag}$ reflector at the back and a $70 \mathrm{~nm} \mathrm{Si}_{3} \mathrm{~N}_{4}$ anti-reflection coating layer at the front (last column). Please note that for the unpatterned film without mirror and anti-reflection coating the calculated absorption is $22 \%$ and thus $7 \%$ higher compared to the measurement, which explains the lower enhancement factor of the simulated structure.

The combination of both gratings further improves the absorption enhancement, yet the improvement is rather modest. The $2 \mathrm{D}$ grating structures were indeed not optimized to yield optimal light-trapping, but were rather chosen to simplify the comparison with a model. Essentially, I used the dual-grating approach as a vehicle to demonstrate the layer-transfer technique. Therefore, if the individual gratings were optimized, higher performances should be possible. Finally, as the propagation lengths are longer in indirect bandgap materials, microcrystalline silicon would benefit more from such a dual-grating approach, but I did not have a source of this material available in order to conduct the comparison. 


\subsubsection{Conclusions}

Using Norland Optical Adhesive 61 (NOA61), I outlined a simple layer transfer technique that allowed me to successfully pattern a $400 \mathrm{~nm}$ thin a-Si:H film on glass from both sides independently. In addition, the measured absorption characteristic of the dual-grating structure stands in very good agreement with numerical simulations. Since the dual-grating shows a higher absorption enhancement over a single grating structure either on top or bottom of the film, the greater performance does also highlight the promise of the dual-grating approach.

My study serves as a proof-of-principle demonstration. If a back-reflector and anti-reflection coating layer were included and the textures designed to yield maximum light absorption in the active silicon material, further improvements will be clearly possible. My layer transfer technique opens a new way to study dual interface gratings with no restrictions on the design parameters of each grating.

\subsection{Plasmonic and diffractive nanostructures for light trapping - an experimental compari- SOn}

In 2008 Catchpole and Polman [131] highlighted the potential benefit of metallic nanoparticles for light trapping. The author's idea is based around the collective oscillations of the conduction electrons in the metal, commonly known as localized surface plasmon modes. Noble metal nanoparticles, if excited at their surface plasmon resonance, can become strong scatterers with scattering crosssectional areas well exceeding the particle's size.

Two years later, the nature article from Atwater and Polman [20] further triggered many new research activities in the field of plasmonics for photovoltaics.

Despite the fact that both diffractive nanostructures and metal nanoparticle scatterers have been widely studied for light trapping, a direct, clear and unbiased comparison between the two has not been carried out yet. 
The difficulty of such a comparison is to keep the absorber material the same, to use an identical anti-reflection coating layer, to account properly for any parasitic effects such as absorption in the metal nanoparticles (or any contact layer) and to retain all electrical properties in order to allow a fair assessment of the lighttrapping mechanism.

Plasmonic solar cells are usually fabricated in a substrate configuration, i.e. the absorber material is deposited onto the metallic nanoparticles. By depositing the absorber material onto the metallic nanoparticles, the particles will add a texture to the active layer and throughout the solar cell device [23, 25], which creates a surface relief that contributes to the scattering properties of the layer. Consequently, the optical effects of the surface interfere with a proper assessment of the plasmonic scattering-properties.

\subsubsection{Methodology}

In order to keep the comparison simple, I decided to use identical absorber material that was structured after deposition and annealing, and used absorption measurements to qualify each light trapping approach. Regarding the light trapping nanostructures, I compared a quasi-random supercell diffractive nanostructure $[92,113]$ with a layer of self-assembled metal nanoparticles on a $240 \mathrm{~nm}$ thin non-hydrogenated amorphous silicon (a-Si) slab on glass; $240 \mathrm{~nm}$ is a thickness close to the value used in thin film solar cells [29].

In a joint project with CNR-IMM Catania, who are specialized in plasmonic structures for light-trapping, each research group fabricated their own design on an identical slab of a-Si, i.e. without the usual rear mirror implemented in plasmonic backreflectors $[25,29,132]$. If the samples were then illuminated from the glass substrate first, the performance of the two different light-trapping concepts can be directly compared - irrespective of anti-reflective, Fabry-Perot interferences or material properties, as these were all kept the same. 

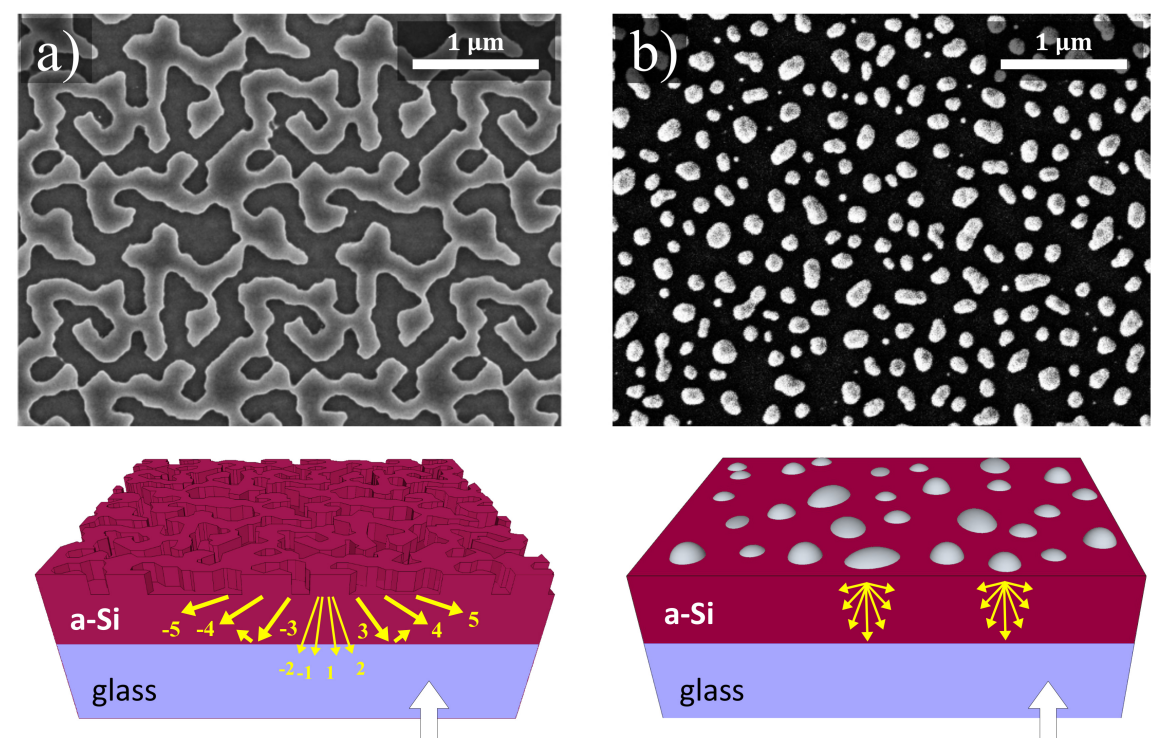

FIG. 4.6: (a) Diffractive 2D quasi-periodic nanostructure, optimized to channel more energy into the higher diffraction orders; (b) plasmonic nanostructure designed to scatter light efficiently into the silicon layer. Both approaches have been realized on the back of a $240 \mathrm{~nm}$ a-Si absorber slab. The white arrows in the bottom sketches indicate the illumination direction.

\subsubsection{The diffractive design}

For the diffractive design, I fabricated the quasi-random supercell structure $[92,113]$ shown in Fig. 4.6a by means of electron beam lithography and reactive ion etching. The supercell's period and nominal etch depth are $1.8 \mu \mathrm{m}$ and $85 \mathrm{~nm}$, respectively.

The supercell concept had previously been demonstrated as one of the most effective light-trapping techniques $[92,133]$, while only relying on a binary grating approach that could be mass-produced using nanoimprint technology [63]. A Fourier analysis (see bottom sketch in Fig. 4.6a) demonstrates the working principle for the high performance of the design: since the structure is able to channel more energy into the higher diffraction orders, more light can couple into the quasi-guided propagating modes of the thin-film, thereby leading to increased optical pathlengths in the absorber slab [58].

Even if the supercell can additionally provide anti-reflection action when used at the front surface, the supercell is here situated on the rear side in order to assess only the diffractive effects, and light enters the absorber through the glasssemiconductor interface first. 


\subsubsection{The plasmonic design}

The plasmonic design consists of self-assembled silver nanoparticles in direct contact with the high-index a-Si layer, an approach that has previously shown strong efficiency enhancements of almost 30\% [25, 134].

Silver $(\mathrm{Ag})$ not only has a low imaginary permittivity, but also a high scattering efficiency in the visible and near-infrared spectrum [135]. In addition, placing the nanoparticles onto the rear side of the absorber was shown to be more advantageous for light trapping than locating them at the front [136]. Thirdly, since the optical density of states in a a semiconductor is higher than in air or silica, it is advantageous to place the scattering centres directly onto the a-Si slab to maximize coupling between the scatterer and the absorber layer [137].

The plasmonic structure is shown in Figure $4.6 \mathrm{~b}$ and was obtained by a postdeposition annealing step of $13 \mathrm{~nm} \mathrm{Ag}$ on a-Si for 1 hour at $450{ }^{\circ} \mathrm{C}$ in $\mathrm{N}_{2}$ atmosphere.

\subsubsection{Results}

\subsubsection{Material properties}

While the optical bandgap $E_{04}$ before annealing was $1.9 \mathrm{eV}$, it was $1.7 \mathrm{eV}$ after the annealing step. The optical band gap of thin film materials, however, varies substantially with deposition conditions. For example, Bakr et al. [138] report optical gaps between 1.8 and $2 \mathrm{eV}$, whereas G. Cody and T. Tiedje et al. [87] cite values between 1.4 and $1.8 \mathrm{eV}$.

To ensure that the a-Si absorber slabs are all identical, a thermal treatment at $450{ }^{\circ} \mathrm{C}$ in $\mathrm{N}_{2}$ atmosphere was carried out before the plasmonic and diffractive nanostructures were realized. The material properties relevant for the study $(n$ and $k$ data) were then carefully measured by variable angle spectroscopic ellipsometry (VASE by Woollam Inc.) and plotted in Fig. 4.7.

In order to check whether the amorphous silicon contains a crystalline phase, Micro-Raman measurements were performed using a HORIBA XploRA instrument with a $532 \mathrm{~nm}$ laser excitation wavelength, x50 magnification and a 2,400 gr/mm grating. 
For the measurements, $2 \mathrm{~mW}$ and $5 \mathrm{~mW}$ optical power were used at the deposited sample (a-Si:H) and the thermal treated sample (a-Si), respectively. The spectra were obtained using 45 3-second acquisitions over which each spectrum was averaged. The material was optically and spectrally verified to be free of any laser damage.

In Fig. 4.8, both the deposited and the annealed film show the broad TO peak of a-Si instead of the typical band of crystalline silicon at $520 \mathrm{~cm}^{-1}$ (used for the calibration of the measurements). Therefore, no crystalline phase is present in the sample.

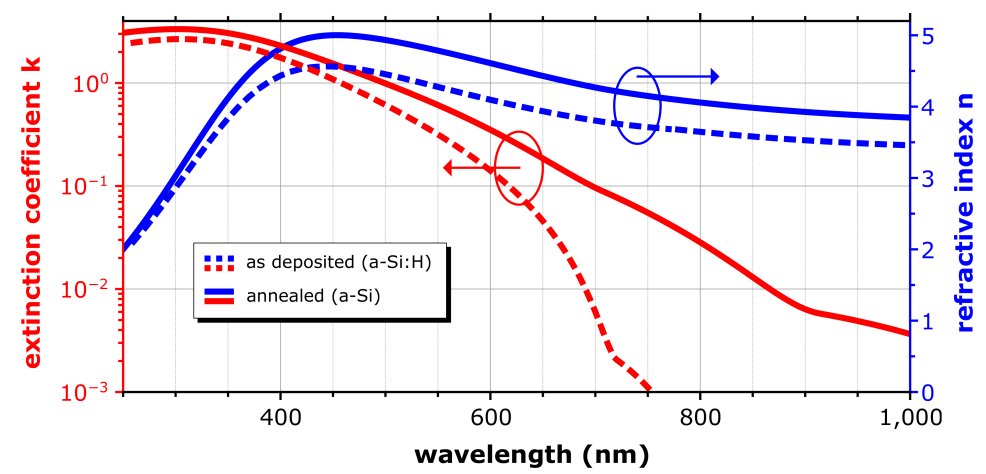

FIG. 4.7: The graphs show the extinction coefficient (red curve, left axis) and the refractive index (blue curve, right axis) of the a-Si, respectively, used in the experiment. The values were determined by variable angle spectroscopic ellipsometry (VASE by Woollam Inc.). The material functions of the a-Si:H, used in section 4.2 , are also shown for comparison.

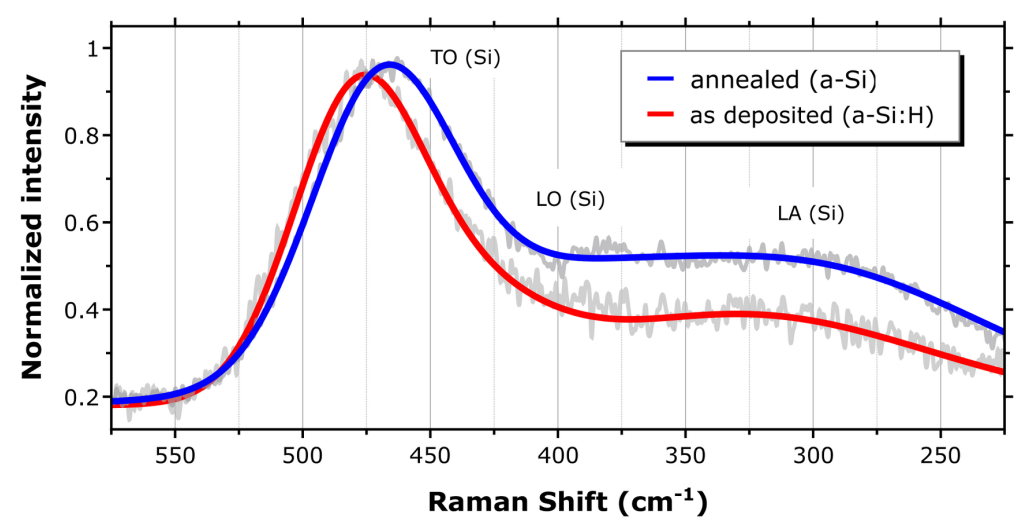

FIG. 4.8: Normalized Raman spectra of the $240 \mathrm{~nm}$ thick hydrogenated a$\mathrm{Si}: \mathrm{H}$ thin film, before (red) and after (blue) a $60 \mathrm{~min}$ post-thermal treatment at $450{ }^{\circ} \mathrm{C}$ in $\mathrm{N}_{2}$ atmosphere. The blue curve, post-annealing, is slightly broadened. This broadening can be explained by the outdiffusion of hydrogen, which induces disorder, while the presence of hydrogen atoms increases the structural order by stabilizing the amorphous network. 


\subsubsection{Absorption measurements}

All samples were carefully characterized by following the method described in section 3.4, and the measured absorption spectra are then plotted in Fig. 4.9.

The quasi-random supercell clearly outperforms the plasmonic nanoparticles in the visible range between 550 and $700 \mathrm{~nm}$, while the opposite is true in the nearinfrared (700 - $1000 \mathrm{~nm})$ where the plasmonic structure clearly causes higher light absorption. The key question is therefore whether the observed absorption is due to stronger scattering of the plasmonic nanoparticles, or whether it is due to undesired parasitic absorption of the metal.

On the one hand, previous studies suggest that for the metal particle sizes involved $(170 \mathrm{~nm} \pm 90 \mathrm{~nm})$ the parasitic absorption becomes minimal in the long wavelength regime $[132,134]$. In fact, according to simple Mie theory the scattering efficiency of a single (spherical) nanoparticle scales with the square of its volume, whereas the absorption efficiency scales only linearly with the particle volume [139]. This argument therefore favours the assumption that the observed high absorption in the near-infrared is mainly due to the strong scattering of plasmonic nanoparticles.

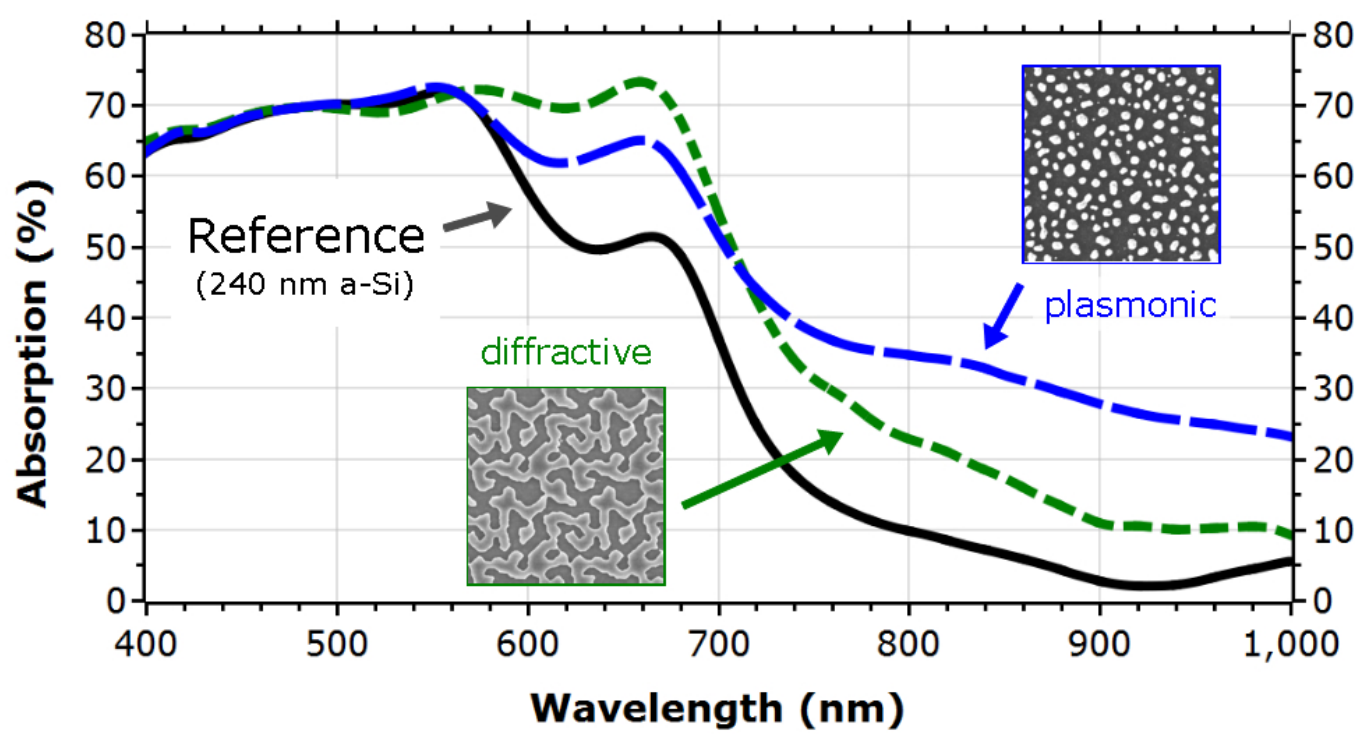

FIG. 4.9: Measured total absorption of the unstructured a-Si slab (black solid line), with added diffractive structure (green short-dashed line) and with added plasmonic structure (blue long-dashed line). 
On the other hand, Schmid et al. [140] showed that the transition from a single nanoparticle to a density of particles already modifies the scattering performances predicted by Mie theory. The high absorption together with the weak wavelength dependence, as observed here between 800 and $1000 \mathrm{~nm}$, thus could also be dominated by the parasitic losses in the metal as it has already been pointed out before [24, 29, 141, 142].

\subsubsection{Discussion}

Mie theory is often intended to provide a first-order estimation of the nanoparticle's performances [134]. While the theory predicts a low parasitic absorption for our particle sizes involved $(170 \mathrm{~nm} \pm 90 \mathrm{~nm})$, the scattered light can come back to the plasmonic particles and be scattered or absorbed again, and again. Consequently, a small parasitic absorption may accumulate to a sizeable absorption overall [143-145]. For example, even if only $10 \%$ of the light is absorbed and $90 \%$ is scattered, at long wavelengths, the light will make many round-trips, interact with the nanoparticles more often and eventually be absorbed parasitically. Therefore, the parasitics have to go up at long wavelengths, as the amorphous silicon becomes transparent (see subsubsection 4.3.2.1).

To quantify the parasitic absorption in plasmonic nanostructures, I therefore suggested to consider the cumulative parasitics that may arise from the multipass nature of trapped light.

I extended the lossy waveguide model outlined in section 2.3 by adding a parasitic loss term for each reflection at the nanoparticle layer. My main assumption here is the inseparability of scattering and parasitic absorption; once the light interacts with the nanoparticle and scatters, it will experience absorption at the same time. This relationship is indeed intrinsic to Mie theory and was also highlighted by Sun and Khurgin [146]. As a result, minimising the parasitic losses is of critical importance, because the desired scattering properties are directly to these undesired parasitic losses. Figure 4.10 schematically illustrates the extended model, which is based on further two intuitive assumptions: 
1. Firstly, since the nanoparticles are broadly distributed and the light is randomized at every reflection, the scattering from the metal nanoparticles will be incoherent. As a result, the (coherent) Fabry-Perot interference fringes around $600-700 \mathrm{~nm}$ are then averaged out. I believe that non-coherence is an acceptable simplification, because averaging these fringes does not affect the integrated short-circuit current.

2. Secondly, the metal nanoparticles scatter Lambertionally. Although nanoparticles are often considered within a point-dipole description, the angular distribution of the scattered light was shown to be similar to the Lambertian case, when Ag nanoparticles were directly situated on the substrate [144]. Lambertian scattering does also take into account light that is coupled into the waveguide by the average scattering angle - in contrast to haze (the non-specular part of reflection), which has no direction.
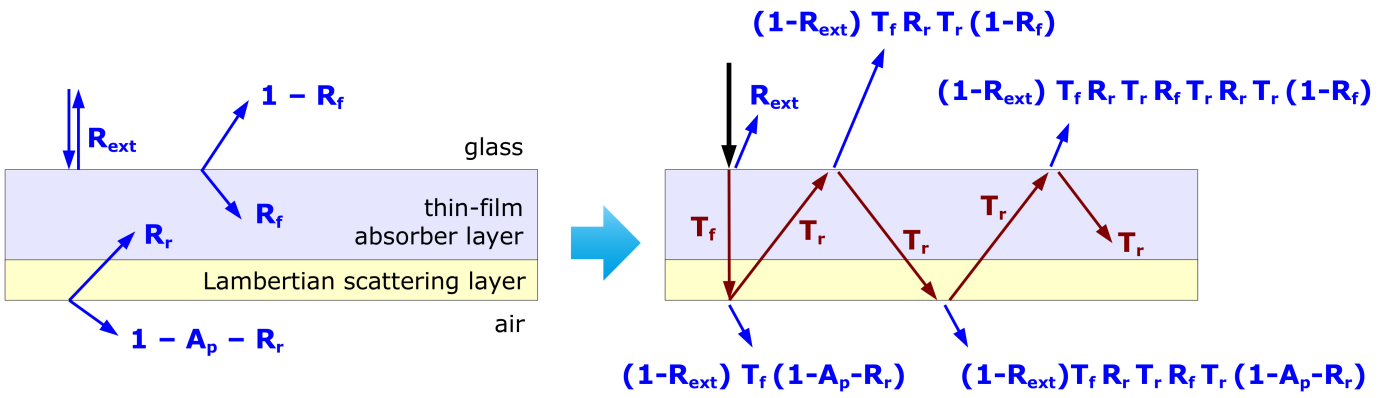

FIG. 4.10: When randomization of light at the scattering layer allows to neglect coherent effects, the propagation of an averaged light ray in a lossy waveguide is described by the external reflection $R_{e x t}$, the internal effective reflectances $R_{f}$ and $R_{r}$, the parasitic metal absorption $A_{p}$ and the attenuated transmission $T_{r}$ with respect to the single-pass traversal $T_{f}$. The incident medium is assumed to be glass, as all measurements were normalized to a reference glass cover slide. 


\subsubsection{Extraction of the parasitic influences}

Using the terms defined in Fig. 4.10, the total absorption $A_{\text {tot }}$ can now be calculated in analogy to Eq. 2.13 via

$$
A_{\text {tot }}^{\text {sim }}=Z \cdot\left[1-T_{f}\left(1-A_{p}-R_{r}\left(1-T_{r}+\left(1-\frac{T_{r}}{T_{f}}\right) \cdot R_{f} T_{r}\right)\right)\right] .
$$

The recirculation factor $Z=\left(1-R_{\text {ext }}\right) /\left(1-R_{r} T_{r} R_{f} T_{r}\right)$ takes into account the attenuation of the average light ray due to the multiple upward and downward reflections at the cladding layers. Since the optical constants $(n, k)$ and the thickness $d$ of the a-Si slab are all known, $T_{f}, T_{r}$ and $R_{f}$ can be readily calculated via Eq. 2.7 and Eq. 2.10, such that all parameters in Eq. 4.1 are determined - except for $R_{r}$ (the effective backreflection from the nanoparticle layer) and $A_{p}$ (the parasitic absorption).

In the following analysis, I will assume that the values for $R_{r}$ and $A_{p}$ are constant across the spectral range of interest. My key argument for spectrally constant fitting parameters is that Mie theory clearly shows that the plasmonic response is flat below $2 \mathrm{eV}$ for particles $>100 \mathrm{~nm}$. In fact, Morawiec and Tan et al. [24, 134] experimentally verified that the resonance is very broad, and almost flat, in the corresponding wavelength range (600-1000 nm).

Using the least square method, I then compare in Fig. 4.11a the difference between the simulated and measured absorption spectrum as a function of the effective backreflection $R_{r}$ and the parasitic absorption $A_{p}$. The fitting outcome allows me to determine the best parameter set $\left(R_{r}, A_{p}\right)=(34 \%, 20 \%)$ directly from the contour plot. Fig. 4.11b finally compares the best fit to the measured data curve.

One could question the uniqueness of the result, because a range of $\left(R_{r}, A_{p}\right)$ values can be used to achieve a good fit in Fig. 4.11b. However, the goal of the analysis is not to precisely quantify the parasitics at each wavelength, but to estimate the overall parasitic losses. These losses are shown as the red curve in Fig. 4.11, and I note that the red curve is very robust against variations (see subsubsection 4.3.1.2): demonstrating little difference between a range of fitting 


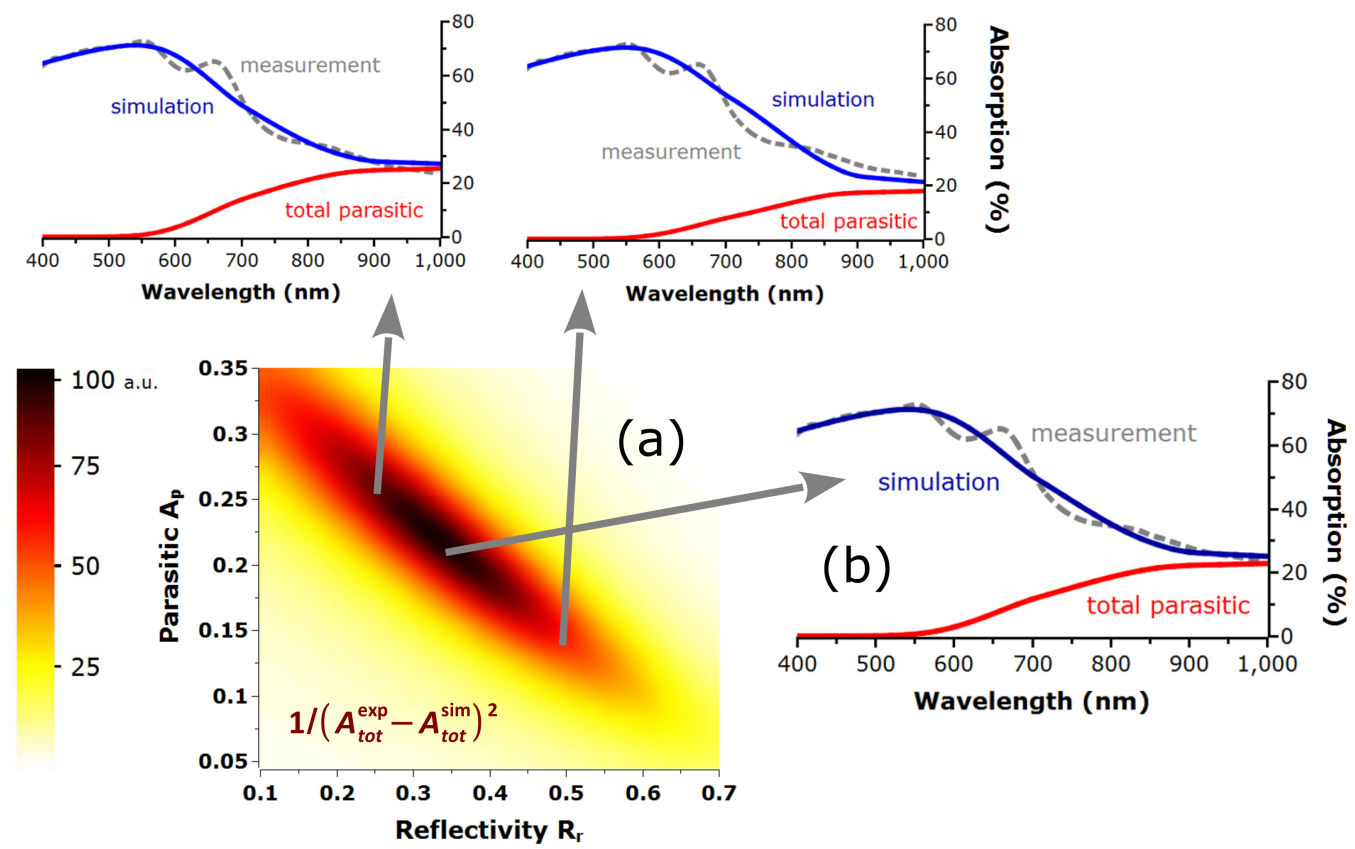

FIG. 4.11: Analysis of the modelled parasitic absorption caused by the metal nanoparticles. (a) Using the least square method, the difference between the simulated and measured total absorption $A_{t o t}$ is shown as a contour plot as a function of the effective backreflection $R_{r}$ and the parasitic absorption $A_{p}$. I used the inverse of the residual $\left(1 / A_{\text {exp }}-A_{\text {sim }}\right)$ as it brings out the differences more clearly. (b) The measurement is then compared to the best fit with $R_{r}=$ $34 \%$ and $A_{p}=20 \%$. Higher or lower values of $R_{r}$ would overestimate either the measured absorption or the parasitics in the visible range, respectively, where most of the light does not reach the backscattering layer or only interacts once with the metal nanoparticles.

parameters shows that the overall conclusion is robust, generally applicable and not restricted to the specific details of the plasmonic structure. Therefore, I varied the $\left(R_{r}, A_{p}\right)$ values in the range of $25 \%<R_{r}<50 \%$ and $13 \%<A_{p}<25 \%$, while maintaining a good fit to the experimental curve.

As a result, I could indeed verify no remarkable differences in the overall parasitic losses, represented by the red curves in Fig. 4.11. This finding can also be understood intuitively: if $R_{r}$ increases, the lightpath will be longer and the light experiences the parasitic absorption more often, hence $R_{r}$ and $A_{p}$ are somewhat interchangeable without affecting the final result.

In order to dispel any final doubts on the generality of the conclusion, I also analysed the measured data by using resonant and wavelength-dependent fittingparameters; maybe the assumption of wavelength-independent scattering and absorption parameters is not correct after all ? To address this question, I assumed 
a Lorentzian lineshape

$$
R_{b}=R_{\text {Si/air }}(\lambda)+\frac{0.45 \cdot 300^{2}}{(\lambda-975)^{2}+300^{2}} \quad A_{p}=\frac{0.12 \cdot 300^{2}}{(\lambda-975)^{2}+300^{2}},
$$

Fig. 4.12 again shows that a very good fit can be achieved, and that the red curve of parasitic absorption is very similar as before (Fig. 4.11). I can thus conclude from the multipass approach that the total parasitic absorption represents a total loss of $12+/-2 \%$, irrespective of the assumptions used.
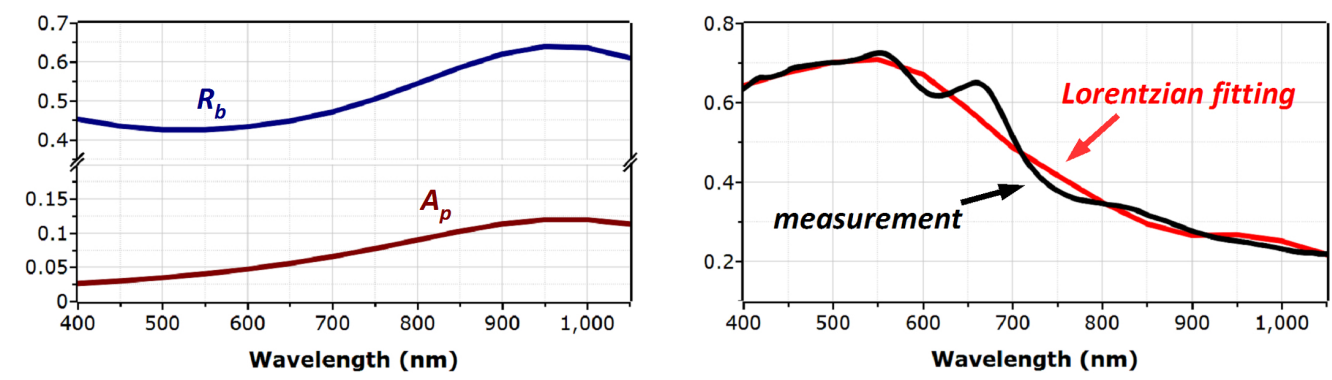

FIG. 4.12: For wavelength-dependent fitting parameters (left), a good agreement with the measurement data (right) is found by assuming the Lorentzian resonance of Eq. 4.2 .

\subsubsection{Comparison of the two different approaches}

Having determined the total parasitic loss, I am now able to isolate the useful absorption from Fig. 4.9, simply by subtracting the parasitic losses from the measured absorption. For comparison, I also included the unstructured reference and an ideal Lambertian backreflector in Fig. 4.13. Over the full wavelength range, the quasi-random supercell now outperforms the plasmonic design.

In order to quantify the differences in performance, I use the LTE introduced in subsection 2.4.2. I first calculated the short-circuit current $J_{s c}$ of the plasmonic device via

$$
J_{s c}=\frac{e}{h c} \cdot \int_{400 \mathrm{~nm}}^{1,000 \mathrm{~nm}} A_{e x p}(\lambda) \cdot\left[1-\frac{A_{p, t o t}(\lambda)}{A_{t o t}(\lambda)}\right] \cdot \lambda \frac{d I}{d \lambda} \cdot d \lambda,
$$




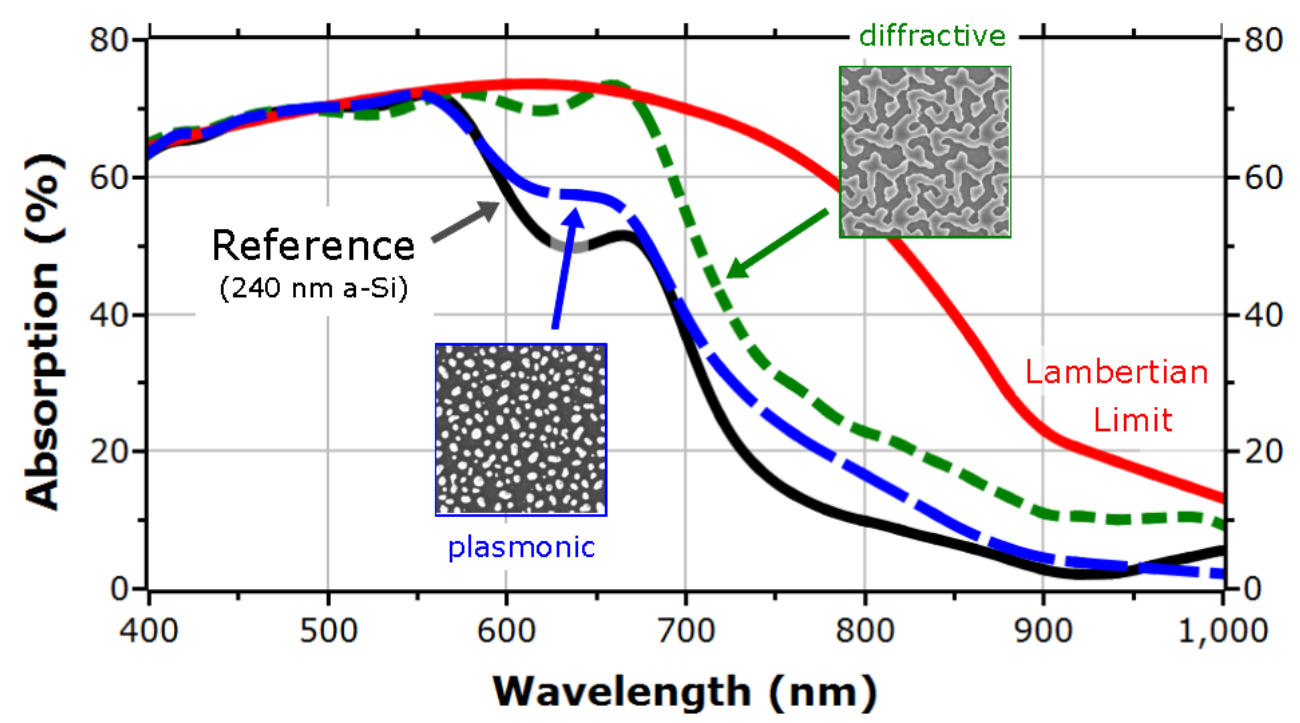

FIG. 4.13: By separating the parasitic contribution from the total absorption in Fig. 4.9, the graphs describe the absorption in silicon only. While the plasmonic structure (blue long-dashed line) can enhance the absorption of an unstructured a-Si slab (black solid line) by 7\%, the diffractive structure (green short-dashed line) is able to do so by $25 \%$. For comparison, the red solid line corresponds to the theoretical absorption of an ideal Lambertian backscatterer.

where I subtracted the total parasitics $A_{p, t o t}$ from the measured absorption $A_{\text {exp }}$. For simplicity, I assumed the internal quantum-efficiency to be unity over the AM1.5 global solar spectrum [89] $d I / d \lambda$. Note that this simplification is artificial and must be dropped when the electric short-circuit current is experimentally assessed, because embedding the comparison into a solar cell would not only require more layers and (optical) interfaces, the assessment would necessarily involve additional effects, such as the electrical, chemical and thermal properties of the device. The impact and interplay of these effects would not only screen the key parameters for light-trapping, but also make it more difficult to extract a clear conclusion in respect to the light-trapping mechanism itself. Here, I do not try to address the question whether the best light-trapping strategy also maximizes the overall power conversion efficiency - this is a different research question beyond the scope of my analysis.

Similarly, I then calculated the implied $J_{s c}$ of the unstructured reference and of the diffractive structure, but without the $A_{p}$ term. The results are listed in Tab. 4.2. 


\begin{tabular}{l|ccr} 
& Absorption & $J_{s c}{ }^{*}$ & LTE \\
\hline unstructured reference & $35.7 \%$ & 13.5 & $0 \%$ \\
plasmonic light-trapping & $38.3 \%$ & 14.6 & $14.2 \%$ \\
diffractive light-trapping & $44.8 \%$ & 17.1 & $47.8 \%$ \\
Lambertian backscatterer & $55.5 \%$ & 21.3 & $\frac{100 \%}{{ }^{*} \text { in } \mathrm{mA} / \mathrm{cm}^{2}}$
\end{tabular}

TAB. 4.2: Comparison of the integrated silicon absorption, the calculated short-circuit current $J_{s c}$ and the LTE [11] for the 400 to $1000 \mathrm{~nm}$ wavelength range.

The $L T E$ related to the diffractive approach is so low, because the supercell was originally designed for light in-coupling and would thus perform better on the front $(L T E=0.68)$. However, the aim of the present study was to quantitatively compare the light-trapping mechanisms by keeping all other parameters as identical as possible, rather than optimising the overall performance of each approach.

\subsubsection{Conclusions}

Plasmonic and diffractive light-trapping using photonic nanostructures has been compared experimentally. Corresponding nanostructures have been placed on the rear side of an absorber slab. Since the incident light will pass through the glass substrate and amorphous silicon layer first, the absorption enhancement can be directly related to the scattering and diffractive properties of the structures. In fact, while previous conclusions have been screened by additional effects or were biased toward a particular approach [23, 24, 147, 148] (with a corresponding loss of generality), using the simplest possible platform enabled me to make a clear and unbiassed comparison of these two light-trapping mechanisms.

For extracting the parasitic metal absorption and the assessment of the lighttrapping performances, I had to the extend the simple analytical model outlined in section 2.3. The analysis turned out to be robust even for a different functional dependence of the parasitic absorption. 
While Ingenito et al. [149] already proposed a similar method to differentiate between the absorptance in silicon and metal, their empirical model only describes a single pass through the material. This can be seen by assuming the silicon to be only weakly absorptive $A_{S i} \sim 0$ and by expressing the metal absorption $A_{A g}$ from Eq. 4 and 5 q.v.

$$
A_{A g}=\left(1-R_{a i r / S i}\right) \cdot \sum_{m=0}^{\infty}\left(R_{@ 1200} R_{S i / a i r}\right)^{m} \cdot\left(1-R_{@ 1200}\right) .
$$

Here, $R_{@ 1200}$ is the measured total reflection at $1200 \mathrm{~nm}$ wavelength, whereas $R_{S i / a i r}$ and $R_{a i r / S i}$ are the external Fresnel reflection at air/Si of Eq. 2.1 and the effective internal back reflection Si/air of Eq. 2.7, respectively.

As the model by Ingenito et al. uses only the first term $(m=0)$, it would not correctly represent the multi-pass nature of a waveguiding system and, therefore, does not facilitate the separate determination of the plasmonic nanoparticles' reflectivity and absorption.

The accurate modelling of non-identical and randomly distributed metal nanoparticles has not been demonstrated yet, because of the difficulties set by the random nature of the particles' shape, size and volume as seen in Fig. 4.6. The lack of order, for example, leads to agglomerations and thus enhanced inter-particle interactions, which are known to reduce the plasmonic resonances [142, 150]. Secondly, both the absorption and the scattering cross section of a nanoparticle usually exceed the particle size, such that a computational cell is difficult to define. Plasmonic resonances are also quite sensitive to the area in contact with the substrate [144] and to defects in the nanoparticle's shell [135]. If a numerical computation were to include all of the above effects, the modelling would become complex or run into convergence problems due to the required fine meshes.

Finally, although thicker metal nanoparticles may scatter even more strongly and absorb less according to Mie theory, the variations would be small. For example, previous works had highlighted that the best performing nanoparticles differ by less than 10\% [134]. Equally, Tan et al. [24] reported that the variation between different size plasmonic nanoparticles only leads to a ca. $10 \%$ variation in haze in reflection in the important $600-900 \mathrm{~nm}$ regime. Therefore, the outcomes in this study would not differ much if better nanoparticles were used. 
The comparison reveals that a quasi-random supercell diffractive grating enhances the silicon absorption compared to an unstructured slab by $25 \%$, whereas randomly distributed silver nanoparticles are only able to do so by less than $10 \%$. Even though the high parasitics are responsible for the poor performance of the silver nanoparticles, they should be less detrimental in a real solar cell configuration, because nanoparticles show a stronger scattering performance when coupled to a rear mirror $[25,132,139]$.

In conclusion, diffractive light trapping outperforms plasmonic light trapping due to the cumulative parasitics arising from multipath interactions at metal nanoparticles.

\subsection{Summary}

By using an optical adhesive to aid the transfer of a silicon thin-film to a new carrier, I developed a simple method for patterning before and after the transfer process without restrictions on the design parameters. In a proof-of-principledemonstration, I etched two different 2D gratings into the top and bottom of a $400 \mathrm{~nm}$ thin-film of hydrogenated amorphous silicon. The absorption measurements could indeed highlight the promise of this approach, because the dual structure showed an appreciable improvement over single gratings patterned either on the top or bottom of the film (see section 4.2).

To study and to quantify the impact of parasitic effects on the silicon absorption enhancement, we decided to compare two different light-trapping mechanisms. In a joint project with CNR-IMM Catania, each research group fabricated their own design on an identical silicon slab. We used a quasi-random diffractive structure, which I had etched into the silicon surface, whereas the research group of Catania focused on self-assembled silver nanoparticles as strong scatterers for the incident light. While Mie theory predicts a low parasitic absorption for the metallic particles, this theory neglects that scattered light can come back to the particles and be scattered or absorbed again. I developed a model for the extraction of the parasitic influence by considering the cumulative parasitics that may arise from 
many round-trips of trapped light (see section 4.3). My analysis turned out to be robust even for different functional dependencies of the parasitic absorption, such that we could generally state that diffractive light-trapping structures tend to be a better choice for photovoltaic applications, because of the cumulative parasitics arising from multipath interactions at the metallic nanoparticles. 


\section{Chapter 5}

\section{Conclusions and outlook}

\subsection{General conclusions and remarks}

A large number of different types of light-trapping structures for thin-film solar cells have been proposed over the last decades. In order to provide a unified, objective and quantitative assessment of the different structures, I have developed a figure-of-merit in chapter 2, called the light-trapping efficiency (LTE), which aims to assess the performance of the nanostructure itself. The LTE separates the light trapping effect from the material, the absorber thickness, the fabrication method and the light-trapping method. The LTE enabled me to compile a ranking list of state-of-the-art proposals in Tab. 2.1 to Tab. 2.3.

Compiling this list allowed me to draw four general design rules, which I will comment on in the following paragraphs.

1. Sub-wavelength structures offer the possibility of suppressing the surface reflection over the useable solar spectrum (see subsection 2.2.2), and can replace, in fact outperform, antireflection coatings.

2. Since the absorber slab acts as a long-pass filter for the incident sunlight, structures on the back side of the absorber only need to address a reduced wavelength range. 
3. Whether a structure presents a periodic or random arrangement of scatterers can be considered as less important than its local geometrical features (see section 2.6).

4. Parasitical loss mechanisms are of critical importance and must be avoided when the active absorber material becomes weakly absorptive.

The first two design rules are addressed by research studies focusing on dualgrating structures. In fact, structuring a thin-film absorber layer from both sides allows us to take advantage of the full benefits that textures can offer. However, theoretical proposals are often limited to supercell-type calculations (where the period on one side is the multiple of that on the other), whereas experimental structures were restricted to those with the same period on both sides (where the structural features of the substrate go all the way through the absorbing material).

By using an optical adhesive to aid the transfer of a silicon thin-film to a new carrier, I developed a simple method for patterning before and after the transfer process without restrictions on the design parameters. In a proof-of-principledemonstration, I etched two different $2 \mathrm{D}$ gratings into the top and bottom of a $400 \mathrm{~nm}$ thin-film of hydrogenated amorphous silicon. The absorption measurements could indeed highlight the promise of this approach, because the dual structure showed an appreciable improvement over single gratings patterned either on the top or bottom of the film (see section 4.2).

The third design rule is derived from the fact that neither completely random nor conpletely periodic structures are able to maximize the absorption enhancement over a broadband spectrum (see section 2.2). The most promising light trapping structures tend to lie between these two domains. This observation can be understood in the framework of diffractive optics:

While the lower diffraction orders generally being more intense in random and periodic designs, the higher diffraction orders are more likely to be totally (internally) reflected at the cladding of a silicon slab, thereby experiencing a longer path length in the absorber layer. As typical structural features form the building blocks of diffraction patterns, their local arrangement thus needs to guarantee a 
suppression of the lower orders so as to enhance diffraction into the higher orders - which in turn will increase the optical absorption due to their longer paths.

The appropriate level of (dis)order in the diffractive structures used in my thesis has been tuned by using its Fourier spectrum as a tool. For the fabrication, I used electron beam lithography and reactive ion etching (see chapter 3). Since electron beam lithography is a pixel by pixel exposure, and thus is relatively slow, I also developed a tool for exploring the technical possibilities offered by nanoimprint lithography in A. The setup I used for characterizing the absorption of my samples is described in section 3.4.

Having gained theoretical and experimental expertise in the design of efficient light trapping structures, I noticed the need for theoretical descriptions to be more comprehensive, as not all relevant optical losses are generally taken into account (fourth design rule). This observation is based on my literature review, because the light trapping efficiency of theoretical structures significantly exceeds that of experimental ones (see Fig. 2.17).

To study and to quantify the impact of parasitic effects on the silicon absorption enhancement, I decided to compare two different light-trapping mechanisms. In a joint project with CNR-IMM Catania, each research group fabricated their own design on an identical silicon slab. I used a quasi-random diffractive structure, which I had etched into the silicon surface, whereas the research group of Catania focused on self-assembled silver nanoparticles as strong scatterers for the incident light. While Mie theory predicts a low parasitic absorption for the metallic particles, this theory neglects that scattered light can come back to the particles and be scattered or absorbed again. I developed a model for the extraction of the parasitic influence by considering the cumulative parasitics that may arise from many round-trips of trapped light (see section 4.3). My analysis turned out to be robust even for different functional dependencies of the parasitic absorption, such that I could generally state that diffractive light-trapping structures tend to be a better choice for photovoltaic applications, because of the cumulative parasitics arising from multipath interactions at the metallic nanoparticles. 
In summary, this thesis outlines the assessment of light-trapping structures, the critical importance of parasitic loss mechanisms and demonstrates a simple layertransfer technique to enable the study of more efficient light-trapping designs.

\subsubsection{Limitations and future work left}

As the electron beam system I used at York was the first ever VOYAGER system produced by Raith, I often encountered exposure issues during my work. Although receiving outstanding support from the company, the regularly recurring software bugs and breakdowns of the pattern exposures slowed the overall lithography process down. Therefore, if I were given a second chance, I probably would have moved on to nanoimprint lithography earlier. For example, time did not allow me to fine-tune the imprint parameters for the replication of structures with simultaneous small and large structural features, such as the quasi-random supercell pattern used in my experiments. Future work thus will need to focus on

1. the influence of the applied pressure during the UV curing step,

2. the matching of the relief height of the stamp to the resist thickness,

3. the determination of the etching rate of AMONIL,

4. the fabrication of bi-layer hard-PDMS/PDMS stamps,

5. the replacement of the current LEDs with a $360 \mathrm{~nm}$ wavelength LED array.

In addition, if time had permitted, I would also have tried to drop cast the nanoresist AMONIL directly onto the stamp (instead of the silicon substrate), because it may make the spinning step redundant, could reduce the necessary amount of resist material and even further facilitate the process. This method is known as reverse nanimprint lithography. Secondly, I would have tried to set up a step-and-repeat imprint process in order to prove the scalability of light-trapping patterns on our simple home-made setup described in section A. 
After the nanolithography process, I used RIE etching to transfer the diffractive nanostructures into the silicon material. However, the kinetic ion energies tend to severely damage the material. While surface defects may be irrelevant in terms of the texture's optical performance, material defects degrade the electrical performance of a thin-film solar cell device. For example, when light trapping structures are made via RIE processes and used in solar cells, the carrier transport can be subject to surface recombination velocities as high as $10^{5} \mathrm{~cm} / \mathrm{s}$ [151]. The need for curing these defects requires efficient surface passivation techniques - solar cells would otherwise not benefit from the absorption enhancement offered by diffractive light-trapping structures.

However, although efficient surface passivation techniques were recently introduced, which allow the curing of defective states, most methods require additional processing steps, reduce the throughput capability and tend to be difficult to integrate into production lines. Why should the combination of nanoimprintlithography and anisotropic wet etching not be an alternative to reactive ion etching then ? The high damage of crystal planes is avoided (less electrical defects), no vacuum equipment is needed (higher throughput and cost-effective), and the optical performance of graded textures has been shown to be advantageous for anti-reflection and light trapping (see subsection 2.2.2).

For these three reasons, I initiated wet-etching tests to explore the feasibility of nanostructuring the silicon surface with tetramethylammonium hydroxide (TMAH). Unfortunately, I had to interrupt these tests, because the mask materials used (flowable oxide, FOx, i.e. spin-on glass; Al and Ni metal layers) turned out to be unsuitable for this purpose. Future work could therefore concentrate to develop this idea further, once one is able to deposit or to grow typical masking materials for silicon, such as silicon nitride or thermal silicon oxide.

However, the need for thin crystalline silicon layers for my experiments also motivated me to use TMAH for the etching of a standard silicon wafer down from $500 \mu \mathrm{m}$ thickness to a slab of tens of microns. Figure 5.1 shows my simple setup for this test along with the resulting outcome. The total etching time may take up to $6 \mathrm{~h}$ at temperatures around $200^{\circ} \mathrm{C}$. 


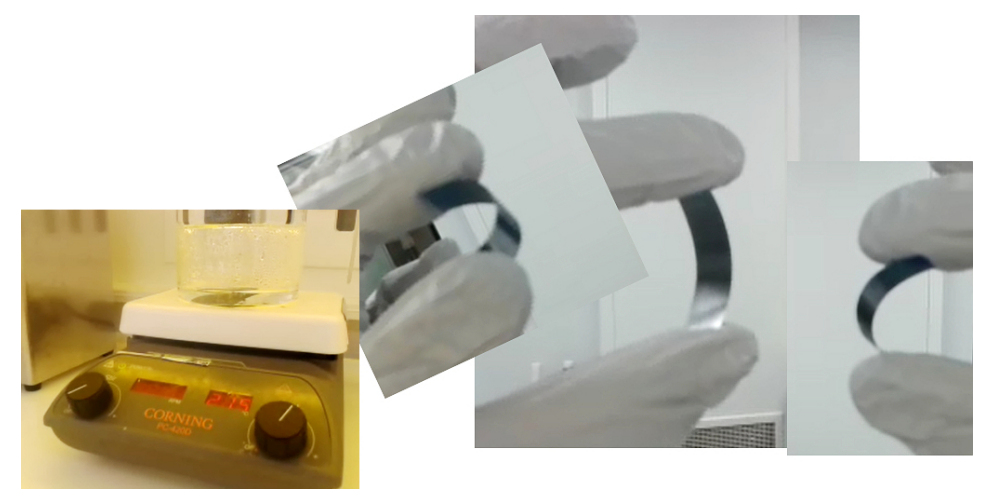

FIG. 5.1: TMAH wet etching allows to thin silicon standard wafers down to a few tens of micrometers. When the sample is left in TMAH at $200^{\circ} \mathrm{C}$ for 6 hours, the thickness is reduced from 500 to $50 \mu \mathrm{m}$ - where the material starts to become flexible.

It was not until recently when I identified the master thesis from Erik Janssen [152] as inspiration for optimizing this etching process, because he reviews five different procedures for thinning substrate wafers down to a few microns. In addition, Janssen also constructed an etching apparatus of Teflon that can be totally submersed in an etching solution of potassium hydroxide $(\mathrm{KOH})$. If time would have permitted, I would have studied Janssen's thesis and optimized the thinning process in light of his suggestions for obtaining a smoother surface roughness, a faster etching rate or to limit the etching attack only to one side of the wafer. The latter may even allow to transfer a high-quality layer of $200 \mathrm{~nm}$ crystaline silicon from the silicon substrate of a standard SOI wafer to a glass carrier for handling.

Future work also needs to focus on the absorption measurement setup. A better collimation of the laser beam is required on the sample, because the measured Fabry-Perot fringes of unstructured thin-films show a reduced dynamic range. In addition, since diffracted light from the sample can be directly reflected towards the entrance port, I would recommend to use a smaller entrance port of a few $\mathrm{mm}^{2}$ area.

Last but not least, this PhD project has been a great experience for me. The developing of theoretical and experimental methods was a comprehensive learning curve in respect of the understanding of light management and of experimental skills in fabrication and characterization of nanostructures. 
I would definitely study light trapping again: although the interplay of light and matter is only the first step in the photovoltaic conversion mechanisms, it must be well understood in order to pursue power efficiencies well above the Shockley-Queisser limit of 30\%. The gained knowledge in this research field therefore becomes important for tandem solar cells that require an intermediate scatterer between the top and bottom cell, i.e. a selective Lambertian reflection of short-wavelength light (top cell) alongside Lambertian transmission of longwavelength light (bottom cell). Such an arrangement could allow to reduce the absorber thickness of both cells and hence facilitate the collection of photogenerated charge carriers.

\subsubsection{Outlook}

The next step would be the realization of a working thin-film silicon solar cell $(<20 \mu \mathrm{m})$ based on the layer-transfer technique and the diffractive supercell structure (see chapter 4). Its realization could allow the study of how lighttrapping influences the power conversion efficiency, i.e. the electrical performance. Here, I will briefly outline one possible fabrication process for such a device, using the steps shown in Fig. 5.2:

1. structuring and coating/passivating the front surface (steps 1-4),

2. passivating the back surface (step 5),

3. fabricating the p-type electrode (steps 6-9),

4. fabricating the n-type electrode (steps 10-13),

5. defining the metal contacts (step 14),

6. applying a spacer layer and a white back reflector (step 15).

Since electrons as the minority-charge carriers are generally able to diffuse longer distances in p-doped crystalline silicon than holes in n-doped $\mathrm{Si}$, the absorber layer can be chosen to be doped lightly p-type already. A lightly doped material also reduces the probability of Auger-recombinations. 
(1)

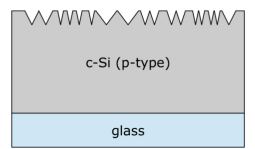

(5)

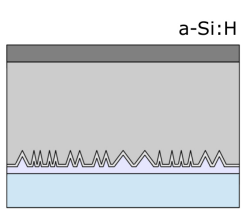

(9)

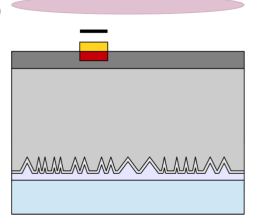

(13)

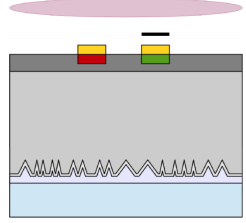

(2)

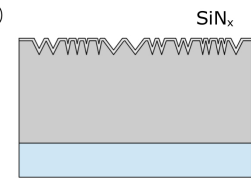

(6)

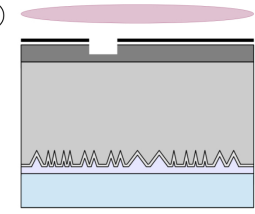

(10)

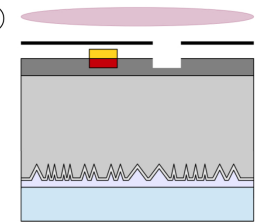

(14)

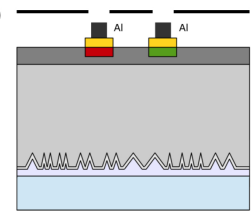

(3)

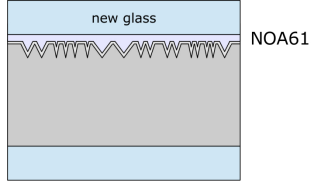

(7)

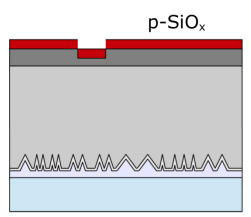

(11)
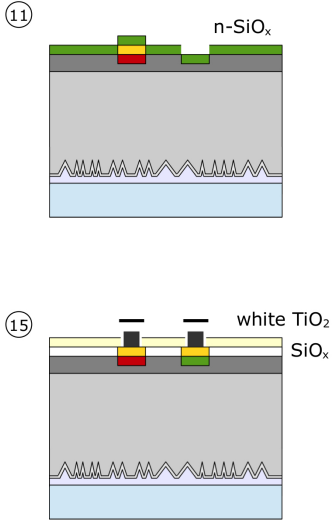

(4)

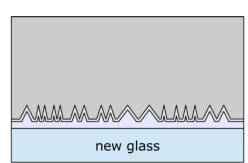

(8)

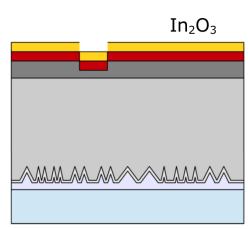

(12)
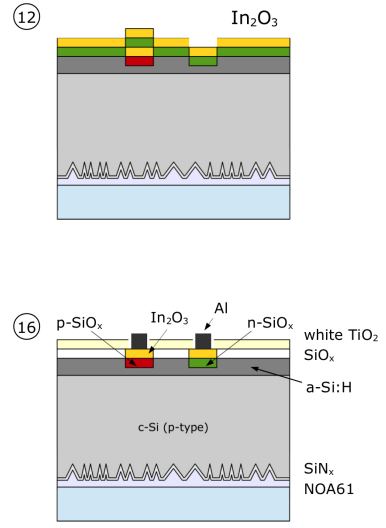

FIG. 5.2: Possible fabrication process for thin-film silicon solar cells (see text).

In order to avoid unnecessary optical losses, thin-film solar cell should use the entire front surface as the active area. Light-trapping structures then can exploit the entire device area at the front, yet both electrical contacts have to be arranged at the back side of the device (in an interdigitated fashion).

Before applying the layer transfer technique, the absorber material must first be structured (step 1) and be passivated, e.g. via the PECVD deposition of $\operatorname{SiN}_{x}$ (step 2), since the foreign glass carrier will not allow the access to the front side any more (step 3). Sunlight, however, is able to pass through the new glass substrate and will be diffracted by the coated supercell structure into the silicon absorber.

After the transfer (step 4), the thin and heavily doped electrodes are applied to the back side. A thin layer of a-Si:H (ca. $10 \mathrm{~nm}$ ) acts as the passivation layer for the back side and as a formation layer for the electrodes. Metal masks allow to selectively dry-etch the contact regions (step 6 and 10) prior the evaporation of the electrode materials (step 7 and 11) and the thin conductive oxide layer (step 8 and 12). Subsequent cleaning steps will address shunting issues (step 9 and 
13) and complete the fabrication of the electrodes. The conductive oxide, here $\mathrm{In}_{2} \mathrm{O}_{3}$, and the sputtered $\mathrm{SiO}_{x}$ (step 15) optically act as a cladding layer for the absorber layer and aim to enhance the total internal reflection of light.

The sputtering of the metal contacts (step 14) and the coating/spray deposition of a white back reflector (step 15) will finalize the fabrication process.

\subsection{Can solar power solve the world's energy crisis ?}

Two important issues I did not address in this thesis so far. First, solar power will only become truly competitive, if the storage problem can be overcome. Once this additional hurdle is solved, I think solar energy can make a significant contribution towards the world's energy production. Second, transport problems can be an issue, because low-latitude countries receive the most hours of bright sunshine during the year, whereas mid- to high-latitude countries have the highest per capita energy consumption.

However, I would like to finish my thesis with a few more general thoughts about our research efforts for a more efficient energy technology. In short, I am asking the question whether the world's energy crisis can be solved by technologies based on solar power.

Energy drives economies, guarantees growth and sustains the existence of billions of people. While human population quadrupled in the last century, our energy consumption increased tenfold in the same period. Some studies even see the increase in population as a consequence of the higher energy consumption, because new technologies have allowed farmers to cultivate increasingly larger fields and to generate greater crop yields. In light of this statement, a $10 \%$ growth of human population would be based on a $50 \%$ greater thermal coal consumption. In fact, China, the country with the fastest growing population, is the largest energy consumer and the world's largest coal producer as well as importer, according to the U.S. Energy Information Administration (EIA). 
In the end, the fast transitions from the agricultural to the digital age have made our living costs more and more energy-intensive.

The continuing population growth might cease when either the energy supply or the space on our planet fails to keep up with our demand for natural capital. If the required space takes into account the amount of biologically productive land and sea area - not only necessary to supply the resources a human population consumes, but also to assimilate associated waste and pollution -, one can define the footprint of human population by its impact on Earth's biocapacity. The global ecological footprint is a standardized figure-of-merit of our demand on the Earth's ecosystems. For example, a footprint of 1 would mean that humanity uses ecological services as quickly as Earth can renew them. The majority of environmental studies, however, agree that we already need a biocapacity greater than our planet to meet our standards of living; we are living too energy intensive: fossil fuels, like coal and petroleum, are identified as the leading causes for our large footprint, but still remain rooted as the response to our rapidly increasing energy demands - even at much higher spot prices and progressively poorer quality (see Fig. 5.3). Ultimately, our strong dependence on fuels has resulted in many political, social and ethical conflicts. I believe these global conflicts will further increase if fossil fuels remain rooted in our society. Consequently, we may are not limited by energy, but by space in support of human sustainability.

The focus recently moved to "green" technologies, that are able to replace the usage of coal and petroleum. In particular, solar power experiences a remarkable revolution, demonstrating new capacities at record installation rates. The enormous potential of solar energy is one of the motivating forces, that drives the new ambitions. England, for example, receives $2.4 \mathrm{kWh} / \mathrm{m}^{2}$ on an average day from sun. Therefore, the sunshine on a London cab can already meet the electricity needs per capita $(8 \mathrm{kWh} /$ day), whereas the sunlight over all England even exceeds world's electricity demands by six times. 
Ironically and despite all the efforts over the last decades, solar power contributes to our net electricity by less than $0.2 \%$ - coal still by over $40 \%$. The promotion of a green technology may be a challenging task, but its success will probably not solve our hedonism on energy: yes, solar power allows to reduce the ecological footprint and so can facilitate humanity's sustainability, but no, I think, it probably will not satisfy the world's screams for energy.

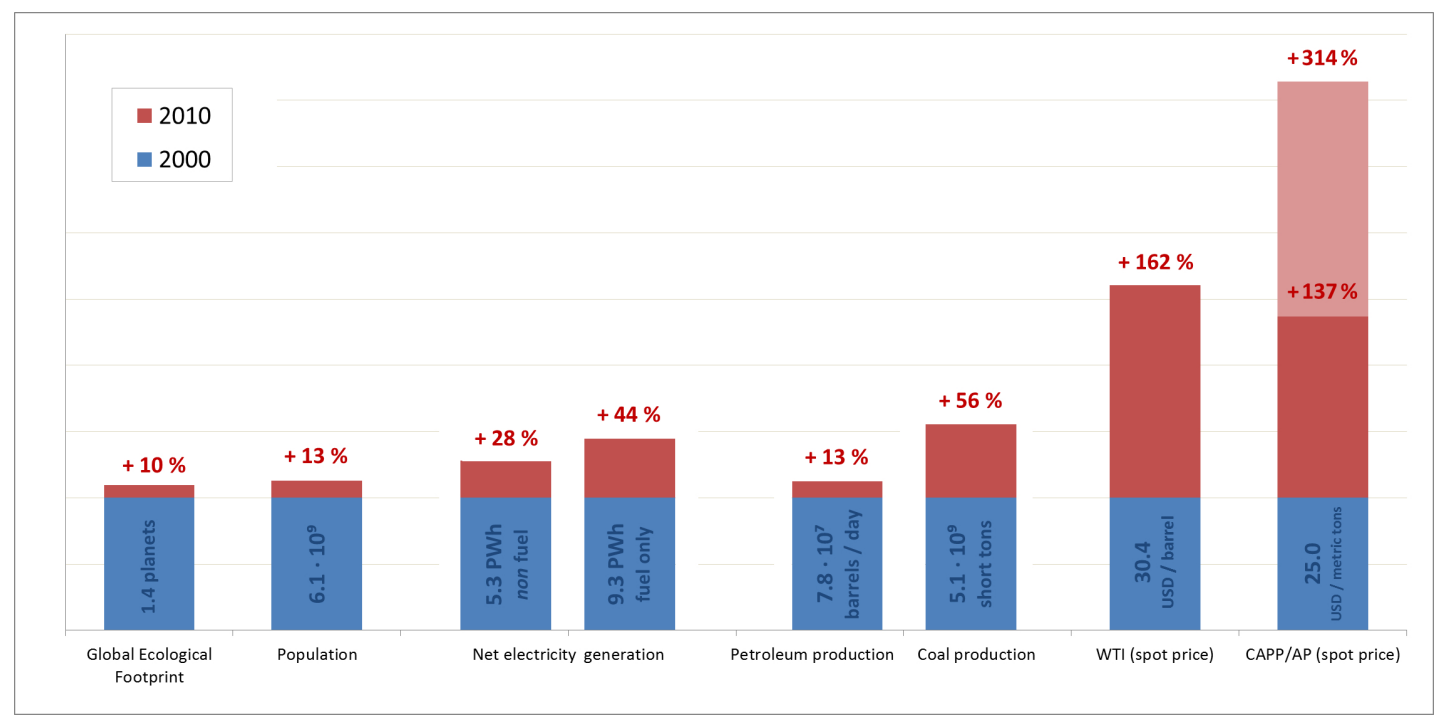

FIG. 5.3: The global ecological footprint is a standardized measure for the human impact on Earth's biocapacity. While in the last decade population and the global footprint grew at almost the same rate, the energy requirements disproportionally increased at much higher costs. Here, the spot prices of the West Texas Intermediate (WTI), of the central Appalachian coal (CAPP) and of the Australian thermal coal (AP) were used in the analysis (see text). 


\section{Appendices}

\section{A A home-made UV nanoimprint tool}

In light of the issues mentioned in subsection 3.2.2, I decided to set up a UV nanoimprint tool, because the technical requirements are less demanding according to Tab. 3.1 on page 64. I decided to use GaN-based LEDs at the required wavelength of $400 \mathrm{~nm}$, they are much cheaper than a conventional UV lamp, they last longer and they do not need to warm up for stability.

\section{A.1 Technical requirements}

Three basic elements conform to the technical requirements of the process flow diagram shown in Fig. A.1.

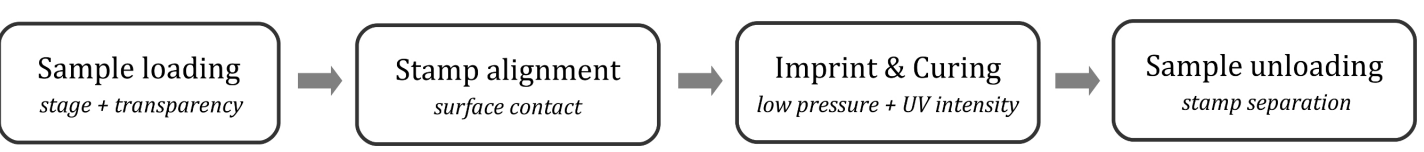

FIG. A.1: As the tool is intended for a single exposure only, with no alignment requirements, the stamp and the resist coated substrate are brought into contact by eye. A transparent stage allows mould (stamp) and polymer layer (resist) to be exposed to UV light from the bottom. GaN-based LEDs (400 nm wavelength) are integrated into the underpart of the tool, while, at the same time, the arrangement is permitting the application of low pressure to the materials by a weight from the top. Following exposure, the sample and stamp remain bound together and must be prised apart, which finishes the nanoimprint process. 
Firstly, a stage is required on the tool for the location of the specimen. The stage has to be transparent to the UV radiation used for curing. Secondly, UV lights needs to cure the resist material. Thirdly, pressure must be applied to the stamp/resist sandwich during the curing process.

The silicon sample must therefore be placed onto a transparent stamp, because pressure can then simply be applied by a weight from the top. Otherwise, extra arrangements would be required for keeping the pressure constant during UV exposure.

Since the LED-array has an emission cone of about $140^{\circ}$, I used an aluminumtunnel of 3 inch diameter in order to funnel the light from the source towards the sample. The Fig. A.2 shows a photograph of the tool. Note that most of the components (LED-array, heat-sink and quartz-disk) were acquired form ebay at a total cost of $<100$ US\$, with some additional help form the mechanical workshop at York.

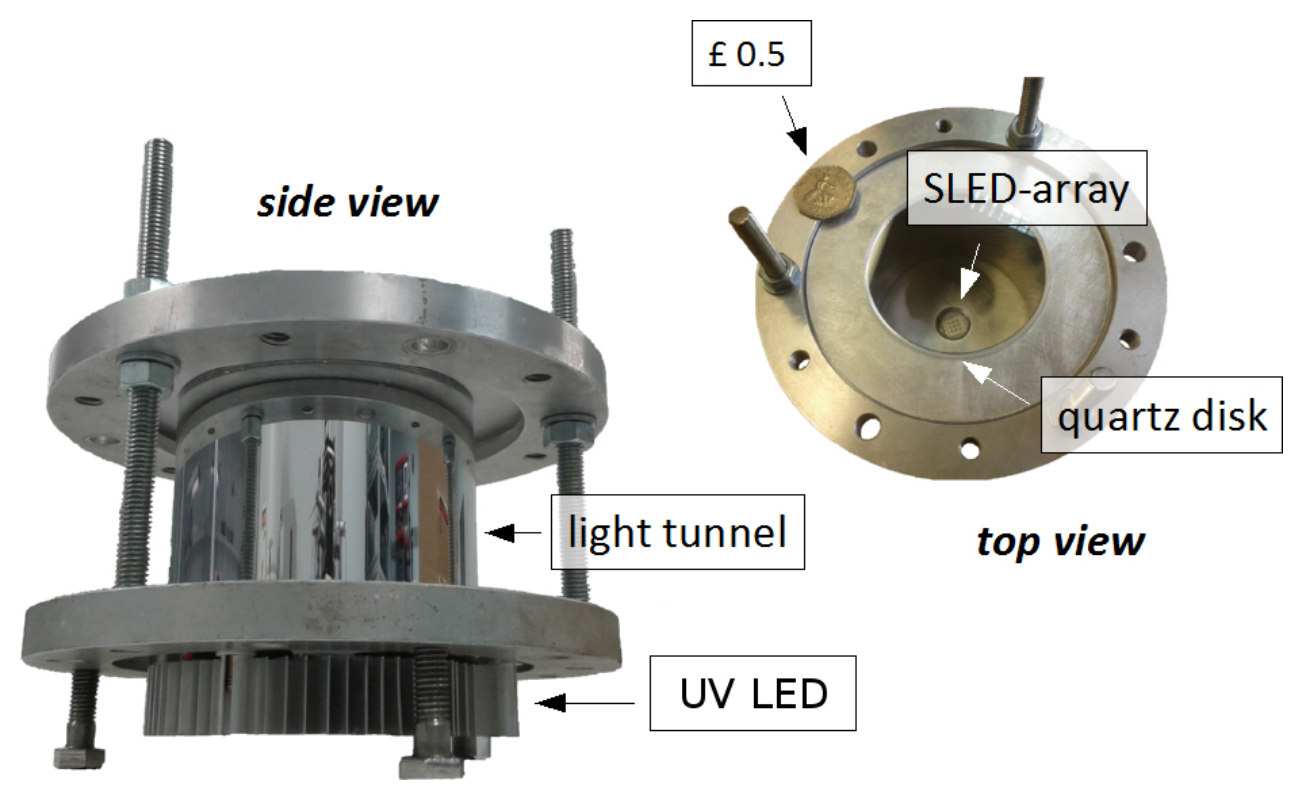

FIG. A.2: The home-made nanoimprint setup is an assembly of a UV-SLED array, a heat sink and a quartz disk (3 inch in diameter). The left and right photograph display the tool from the side and the top, respectively. A 50 pence coin is also shown as a size reference. The light tunnel is made of a glass tube with aluminium evaporated on the inside surface in order to direct the diffusive light from the SLED array towards the quartz disk. The electrical and optical characteristics of the setup are listed in Tab. A.1. 


\section{A.2 Technical specifications of the UV tool}

The specifications of the home-made UV nanoimprint tool are summarized in

Tab. A.1; the optical characteristics were measured in the department. Using three different methods, i.e. a PIN diode, a power meter and a spectrum analyzer, I could accurately determine the optical output power shown in the right hand side of Tab. A.1.

At the sample plane, I noticed that the illumination is not uniform, and drops from $28 \mathrm{~mW} / \mathrm{cm}^{2}$ at the center towards $14 \mathrm{~mW} / \mathrm{cm}^{2}$ at the border. The structured region of the template, however, is always less than $1 \mathrm{~cm}^{2}$, such that the imprint process remains unaffected by the non-uniformity if the structured area is placed in the central part of the quartz-disk. In addition, the radiance on the quartz-disk is less than $30 \mathrm{~mJ} / \mathrm{cm}^{2}$ per second, which is very low compared to the typical curing dose of $1 \mathrm{~J} / \mathrm{cm}^{2}$ according to Tab. 3.1. Therefore, the nonuniformity does not result in internal stress in the nanoresist (see page 65) even for larger structured areas.

\begin{tabular}{lr}
\hline \hline Forward Voltage: & $27-30.6 \mathrm{~V}$ \\
Forward Current: & $350 \mathrm{~mA}$ \\
Input power: & $10 \mathrm{~W}$ \\
Central wavelength: & $395-400 \mathrm{~nm}$ \\
Intensity on disk: & $14-28 \mathrm{~mW} / \mathrm{cm}^{2}$
\end{tabular}

TAB. A.1: Specifications of the LED array used in the homemade UV nanoimprint tool. The electrical data were taken from the datasheet, the optical characteristics were accurately measured in the department. The intensity on the 3 inch quartz disk drops from $100 \%$ at the center towards $50 \%$ at the border, c.f. Fig. A.2. 


\section{A.3 Testing the tool by photo lithography}

In order to verify the operation of the tool, I conducted two tests:

1. Shipley Microposit S1813 requires an exposure dose of $140 \mathrm{~mJ} / \mathrm{cm}^{2}$. The resist is optimised for $436 \mathrm{~nm}$ (g-line), which is close to the emission from the LED array. An exposure time of 7 seconds was sufficient to expose the resist, which corresponds to an effective intensity of $20 \mathrm{~mW} / \mathrm{cm}^{2}$, and hence within the measured range.

2. Norland Optical Adhesive NOA61 requires an exposure dose of $3 \mathrm{~J} / \mathrm{cm}^{2}$, which is 20 times higher than for S1813. I therefore tried to glue two microscope slides together and used a 3 minute exposure time for curing.

Both trials were successful, despite the fact that the exposure properties of S1813 and NOA61 are optimized for use at $436 \mathrm{~nm}$ (g-line) and $365 \mathrm{~nm}$ (i-line), respectively. Therefore, the curing wavelengths will refer to recommendations, as the materials must have a far broader UV sensitivity.

\section{A.4 The stamp materials}

The stamp is a central part of the imprint process, because it must retain and consistently replicate the same structures many times. The materials used for the fabrication of stamps have to meet the following criteria:

1. Stamps have to be transparent to UV light.

2. Stamps must be chemically compatible with the resist material: a stamp should not get swollen by the liquid resist, which could affect the pattern profile or duration of the stamp, or be penetrable for oxygen, which may impede the solidification process of the resist.

3. The easy detachment of a stamp from a hardened polymer layer needs to be guaranteed to prevent pattern damages (caused by a rip-off) and contamination (caused by sticky residues). 
4. Ideally, a stamp would conform to any waviness of the substrate. While small air bubbles, trapped in the cavities of the stamp, will likely dissolve in the resist material, larger air bubbles may remain and significantly affect the imprint result.

In addition, the inclusion of particles between stamp and resist must be eliminated, since these will distort the imprinted replica.

Microscope glass slides comply with criteria (1) and (2), and the treatment of a patterned glass-surface with fluorinated silane groups [123] could also address requirement (3). However, e-beam lithography on non-conducting oxides is generally complicated by the poor charge dissipation and need for high resolution. While the former obliges a high clearing dose $\left(>700 \mu \mathrm{C} / \mathrm{cm}^{2}\right)$, the latter prohibits the usage of a large aperture. Correspondingly, exposures could become too time-intensive. Since the etching of nanostructures into glass would furthermore require a parameter study on the RIE, I chose to focus on stamp materials that offer a faster turnaround time from design to pattern.

I had to try two approaches to find out the one that worked:

a) Instead of using the glass directly, it serves as a substrate for spin-coating the stamp material that can fulfil the criteria (1-4).

b) Using a flexible material such as poly(dimethyl)siloxane (PDMS).

For the first approach (a), which turned out to fail, I used the Fluorolink products MD500 and MD700 from Solvay Solexis [153]. MD500 is a UV curable polyether, which acts as the transparent fluorinated cast (1), whereas MD700 is applied as a primer, providing mechanical strength and a strong adhesion of MD500 to the glass substrate (3). The Fluorolink products then only had to be tested against criteria (2), i.e. the chemical compatibility between the stamp and the resist. The compatibility test, however, failed with the available resist (Microresist UVCur21), because the stamp material MD500 is permeable to oxygen, inhibiting the resist's solidification. 
For the second approach (b), which turned out to work, PDMS is first cast and cured against a template. Such rubber-elastic stamps not only conform to any waviness of the substrate (4); if the PDMS stamp is placed in contact with a liquid resist, capillary forces tend to pull the stamp into the resist, thereby minimizing the possibility of eventual structural deformations during the UV curing process. While PDMS is transparent and therefore complies with criterion (1), the swelling of PDMS in resist solutions may affect the durability of the stamp and could violate criterion (2), i.e. the chemical compatibility between the stamp and the resist. However, this compatibility issue was solved by the German company AMO that developed a UV nanoresist material (AMONIL) especially for PDMS stamps.

In addition, Odom et al. [154] introduced an imprinting method based on a composite bi-layer stamp of hard-PDMS/PDMS. A thin layer of hard-PDMS enables imprint capabilities down to the $20 \mathrm{~nm}$ range [155], while the thick PDMS layer ensures the global flexibility by its lower Young modulus. The bi-layer stamp method therefore ensures the easy detachment from the cured resist, i.e. criterion (3). Hamouda et al. [156] could even prove the externally applied pressure to be dispensable, which relaxes the technical requirements for the UV curing process.

Using a composite bi-layer stamp of hard-PDMS/PDMS and the nanoresist AMONIL (MMS4), mentioned above, I was able to successfully imprint silicon substrates, which will be outlined in more detail next. 


\section{A.5 Testing the tool by nanoimprint lithography}

The nanoimprint process is conducted with a composite bi-layer stamp of hardPDMS/PDMS, the resist AMONIL (MMS4) from AMO and a silicon substrate. The successful process is illustrated in Fig. A.3 and consists of three basic steps:

a) In order to facilitate the peeling off and to ensure no deformations of the imprinted nanostructures, the PDMS stamp is treated with a fluorinated silane group, which acts similarly as a Teflon coating [123].

b) Since the resist will be used as a mask to transfer the imprinted pattern into a silicon substrate by etching, a $200 \mathrm{~nm}$ thick layer of AMONIL (MMS4) resist is spin-coated onto a silicon substrate. As an additional precaution against the peel-off issue, I also briefly apply a gentle oxygen plasma to the silicon surface just before spinning the resist; this treatment mediates a strong binding between silicon and resist.

c) The next step is the contact with the stamp, because AMONIL does not require a baking step in between the spinning and the UV curing process. After locating the stamp in the central part of the quartz disk of the UV tool, the silicon sample is carefully placed on top of the stamp with the resist coated side facing downwards. A gentle press - followed by the positioning of a small weight $(100 \mathrm{~g})$ on the back side of the silicon substrate - finishes the merging process of stamp and resist layer.

a)

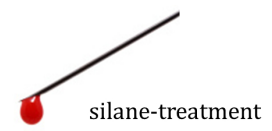

hard-PDMS/PDMS stamp b)

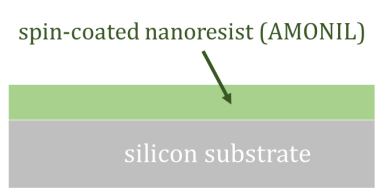

c)

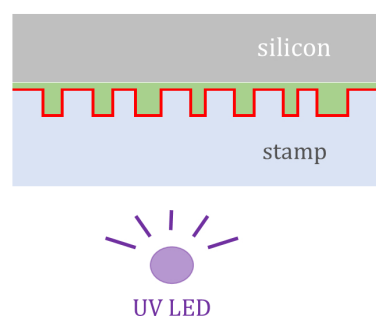

FIG. A.3: a) A compositite bi-layer stamp of hard-PDMS/PDMS is made hydrophobic by deposition of a silane layer, which acts similarly as a Teflon coating and therefore effectively addresses the peel-off issue. b) A thin-layer of AMONIL resist is afterwards spin-coated onto a silicon substrate. The resist may later be used as a mask to transfer the imprinted pattern into the silicon by etching. c) AMONIL is gently pressed into the PDMS stamp and then cured under UV light. 
The UV light exposure completes the nanoimprint process. However, while the LEDs emit UV light at $400 \mathrm{~nm}$ wavelength, AMONIL is designed for UV-curing at $320 \mathrm{~nm}$ and requires an exposure dose of $2,000 \mathrm{~mJ} / \mathrm{cm}^{2}$. If I simply calculate the overlap integral between the light source (see Tab. A.1) and the sensitivity curve of AMONIL (taken from the datasheet), I find a correction factor of 20. Therefore, the required exposure time extends to $2,000 \mathrm{~mJ} / 1.5 \mathrm{~mW} \approx 20 \mathrm{~min}$ on my tool. Since applying higher doses or broader UV-wavelength ranges would neither affect the imprint quality nor the properties of the cured polymer according to the manufacturer, the AMONIL is instead exposed for 30 minutes as a precautionary measure.

After exposure, the silicon substrate and PDMS stamp will remain stuck together, but they can easily be separated from each other by manually peeling the flexible PDMS stamp from the silicon sample. Figure A.4a shows the outcome of the nanoimprint lithography process.

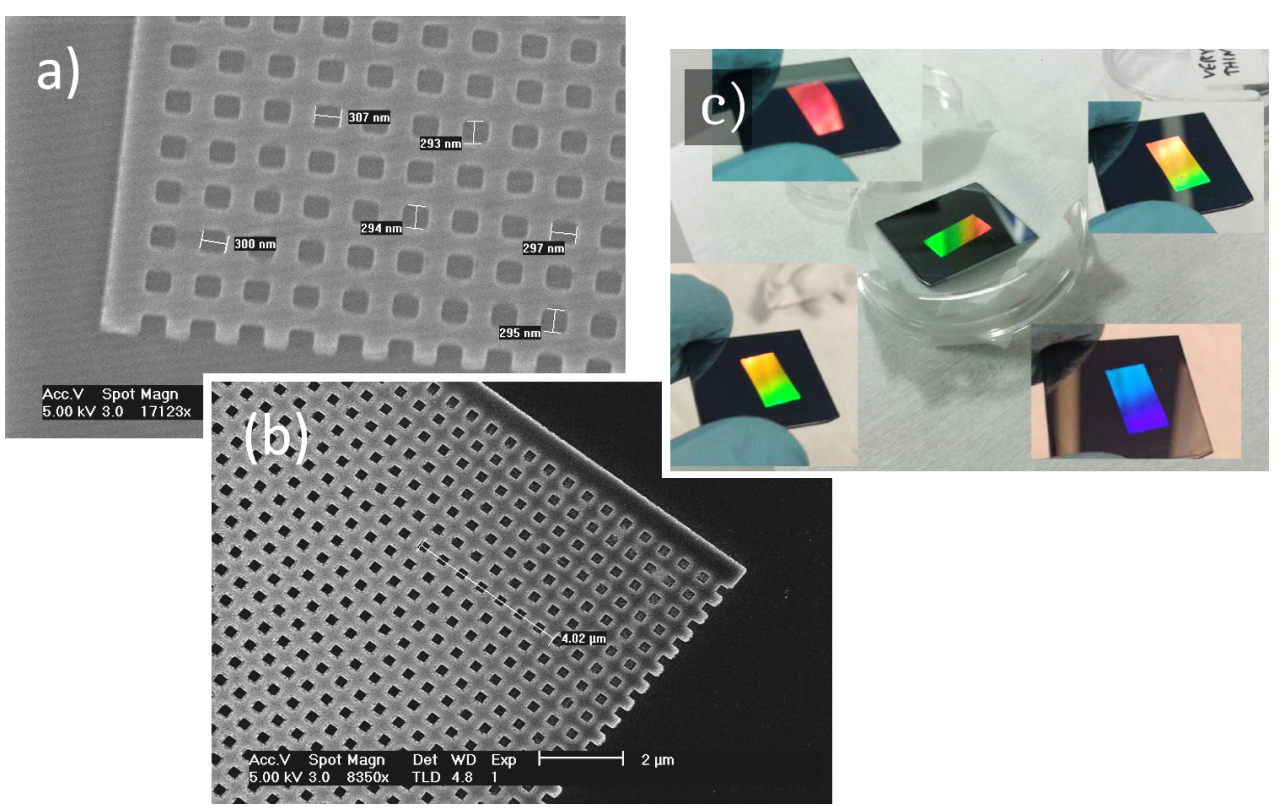

FIG. A.4: The first results of the UV home-made nanoimprint tool presented in Fig. A.2. The above pictures show a Si photonic crystal with period $600 \mathrm{~nm}$ (a) right after the demoulding step and (b) after RIE etching. The nanoresist AMONIL exhibits good masking properties. Picture (c) shows the imprinted sample from different angles to highlight the diffractive properties of the photonic structure. 
The final step is the transfer of the pattern into the silicon substrate. The sample is therefore etched for 1 minute via RIE, then cleaned in a fresh prepared piranha solution $\left(\mathrm{H}_{2} \mathrm{SO}_{4}: \mathrm{H}_{2} \mathrm{O}_{2}=3: 1\right)$ and again inspected (see Fig. A.4b). AMONIL tends to exhibit good masking properties, because the test structure was successfully transferred from the resist into the silicon substrate.

In conclusion, the tool can be used for UV nanoimprint lithography. Using composite bi-layer stamps of hard-PDMS/PDMS along with the nanoresist AMONIL allows the fabrication of nanostructures in silicon. The tool also allowed me to structure the surface of unpolished silicon substrates with $160 \mu \mathrm{m}$ thickness.

\section{A.6 Concluding remarks}

Many requirements are set to UV nanoresists as spin-on solutions; a composition of functional compounds and monomer mixtures is not only fine-tuned to yield a special combination of chemical/physical properties before and after photoinitiation, the components themselves must also be chemical compatible with each other.

Table A.2 overviews the key requisites for a UV nanoresist. All listed criteria are met by the AMONIL resist from AMO in combination with a composite bi-layer stamp of hard-PDMS/PDMS.

\begin{tabular}{lr}
\hline \hline before photoinitiation & after photoinitiation \\
\hline liquid spin-on solution & adhesive to the substrate \\
adequate thickness range & tensile strength \\
low exposure dose & integrity of patterns \\
coating with capillary action & anti-sticking to the stamp \\
insensitivity to oxygen inhibition & etching resistance \\
\hline
\end{tabular}

TAB. A.2: Many chemical and physical properties must be met by a UV nanoresist before (left column) as well as after photoinitiation (right column), while its chemical components have to be compatible with each other at the same time. Since the combination of these properties tends to be difficult to meet, suitable nanoresists tend to be expensive, e.g. AMONIL $\sim 6 € / \mathrm{ml}$. 


\section{B Absorption measurement setup}

In order to characterise my samples, I built a bespoke setup. The following subsection B.1 provides detailed background information about this setup.

\section{B.1 Design considerations}

For accurate absorption measurements, the total reflection $R$ and total transmission $T$ of a sample should be determined at the same time. However, standard spectrophotometers only allow to measure $R$ and $T$ one after the other and by repositioning the sample. When the spectra are obtained in sequence, the measurements could differ either in the angle of incidence or in the illuminated area of the sample, and therefore resulting in artefacts and inaccurate data sets, e.g. the absorption exceeds 1 or falls below 0 . In order to avoid such artefacts, I modified a standard integrating sphere such that $R$ and $T$ are detected simultaneously.

\section{B.1.1 The integrating sphere}

Integrating spheres are designed to spatially increase the net radiant flux by multiple reflections inside. The higher the sphere surface reflectance (here Lambertian Spectraflect ${ }^{\circledR}$ coating) and the smaller the port fraction, the greater the radiance. The radiance is typically enhanced by 10 to 30 times in a sphere with respect to a planar diffuser of the same surface area [157], yet decreases with larger diameters.

Since the sample is mounted in the center of the sphere, the arrangement as such will affect the overall throughput of the integrating sphere (see Fig. B.1). The change in sphere throughput - caused by mounting the sample in the center of the sphere - is known as substitution error and is due to specular reflections between the sample and the light in the sphere, even after the beam's first strike on the sample [158]. However, this error can be corrected, if neither the sample is too large nor the sphere diameter too small. 
Regarding the choice of integrating sphere, there is a trade-off between a small substitution error (requires a large sphere diameter) and a high radiance (requires a small sphere diameter). According to the E903 international standard of the American Society for Testing and Materials (ASTM), the specimen area should be limited to $1 \%$ of the surface area of the sphere. While this choice was not made clear in the ASTM guidelines, the definition closely corresponds to the typical port fraction. The substitution error can then be understood as if a substitutional radiance loss was caused by an additional port on the sphere.

Since my samples are no larger than $2 \mathrm{~cm}^{2}$, this recommendation suggests a diameter of at least $8 \mathrm{~cm}$. I therefore opted for a 4 inch integrating sphere $(10 \mathrm{~cm}$ diameter). While a 2 inch sphere would have been too small, a 6 inch system would yield a lower radiant flux at the detector port.
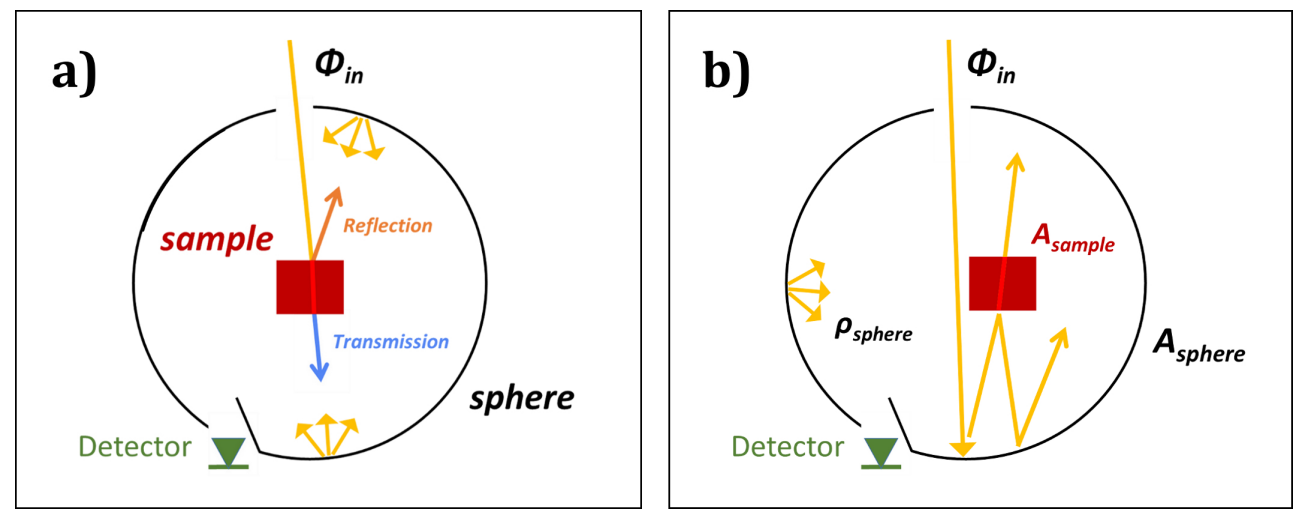

FIG. B.1: Integrating spheres are designed to spatially increase the incident radiant flux $\Phi_{\text {in }}$ by multiple reflections inside the sphere. For absorption measurements, the test sample is mounted in the center, such that both the total reflection and total transmission are detected simultaneously (a). However, the light in the sphere can interact with the area of the specimen $A_{\text {sample, even }}$ after the beam's first strike on the sample, and hence significantly change the optical flux at the detector port. If the area of the sample $A_{\text {sample }}$ is much smaller than the area of the sphere $A_{\text {sphere }}$, i.e. $A_{\text {sample }} \leq 0.01 \cdot A_{\text {sphere }}$, these interactions can be corrected by taking a reference spectrum. The sample must therefore be kept out of the direct line of sight, as indicated in (b). A baffle further prevents any direct irradiation of the detector and ensures a hemispherical field-of-view. 
In order to minimise artefacts while achieving a high throughput, the requirements on the integrating sphere are therefore:

1. $8-10 \mathrm{~cm}$ diameter size

2. Integration of a center mount sample holder

3. Introduction of a chalk baffle in front of the detector to prevent any direct irradiation

4. Reduction of the port fraction by minimizing the size of the entrance port

5. Mounting the photodetector directly onto a port reducer

\section{B.1.2 The monochromator}

Light-trapping, anti-reflection action and in-coupling of photons are wavelength dependent mechanisms. Light-trapping by diffraction particularly relies on the interaction between the light waves and the topographical surface features. Therefore, useful absorption measurements require the performance of spectrographs with nm-resolution. I will discuss two options for the setup:

1. A CCD spectrometer, which is able to detect the incident light intensity versus wavelength at the detector port.

2. A monochromator, which is able to transmit a tuneable narrow band of wavelengths to the entrance port.

The first option, which I decided not to use, is to spectrally analyse the output light with a spectrometer. The USB4000 from Ocean Optics offers an elegant solution, as it uses a linear CCD array for the spectral analysis and features a wavelength resolution of $\Delta \lambda=1.4 \mathrm{~nm}$.

However, the small pixels of the detector $\left(8 \times 20 \mu \mathrm{m}^{2}\right)$ may be too small for our application, given the low radiant flux inside the sphere and the diffuse light conditions; the USB4000, like any grating spectrometer, requires a collimated input light beam. In addition, the spectral sensitivity of the CCD sensor is also markedly blue-shifted with respect to a standard silicon photodiode, which may limit the detection of near-infrared light, as shown in Fig. B.2. 


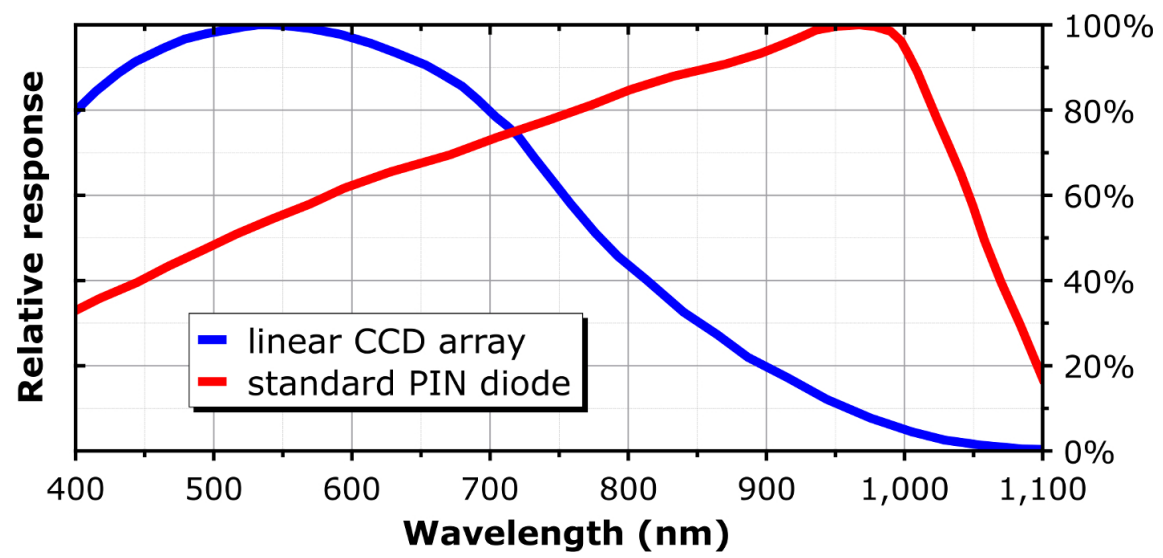

FIG. B.2: Spectral sensitivity of the linear CCD array used in the USB4000 (blue curve) differs from a standard photoreceiver PDA100 (red curve) by a considerable blue shift, even when the same material is used. The optical effect of MOS-capacitors and passivating coating-layers on a CCD chip tends to influence the spectral response. In contrast, the spectral dependency of standard photodiodes is mainly determined by the absorption coefficient of the bulk material, because of the general absence of electronic surface structures on the active area.

The second option, which I opted for, is to spectrally select the input light with a grating monochromator and to combine this with a very sensitive detector on the output side; the detector does not require collimated light, so is more suited to operating with an integrating sphere. The advantages of monochromators are the sub-nm optical resolution, due to focal lengths far greater than $100 \mathrm{~mm}$, and the possibility of analyzing multiple spectral ranges, as usually more than one grating can be used.

Even if the monochromator increases the complexity of the setup, the apparatus adds a valuable degree of flexibility to the experiment. I operated the apparatus as a tunable light source together with a SM-30 supercontinuum white light source from LEUKOS. The spectrum of the SM-30 is shown in Fig. B.3.

For silicon absorption measurements, the monochromator needs to perform best from the UV to the silicon bandgap. Consequently, the required diffraction grating must cover the $400-1100 \mathrm{~nm}$ range and be blazed in the visible spectrum. Blazing means that the grating allows to concentrate a large percentage of the incident energy into a specific diffraction order.

In order to avoid overlap between different diffracted orders of the grating, the selected wavelength is reflected to the exit slit as the first diffraction order, while 
a longpass filter is inserted in the optical path for limiting the width of other diffraction orders. Scanning a spectrum is then accomplished by rotating the grating with a motor.

I chose the diffraction grating from Richardson Optics shown in Fig. B.4, as it has a high diffraction efficiency over a broad spectral range.

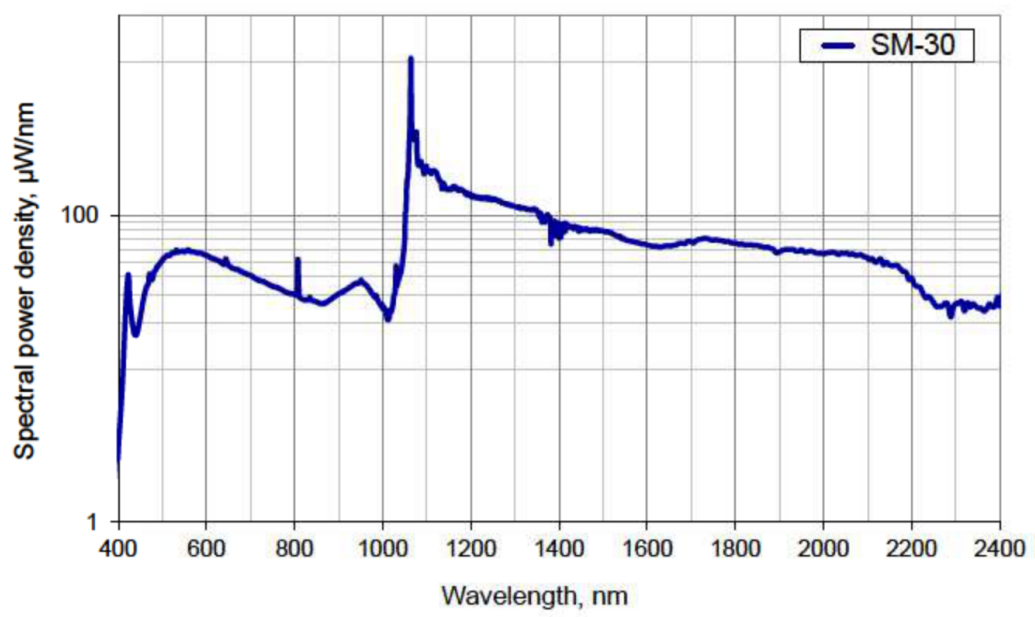

FIG. B.3: The SM-30 supercontinuum white light laser source from LEUKOS uses the non-linear effects of a photonic crystal fiber to generate a broadband spectrum. The seed repetition rate is $30 \mathrm{kHz}$ at the pump $1064 \mathrm{~nm}$ wavelength of a Nd:YAG laser ( $1 \mathrm{~ns}$ pulse width), hence the apparent spike at 1,064 nm.

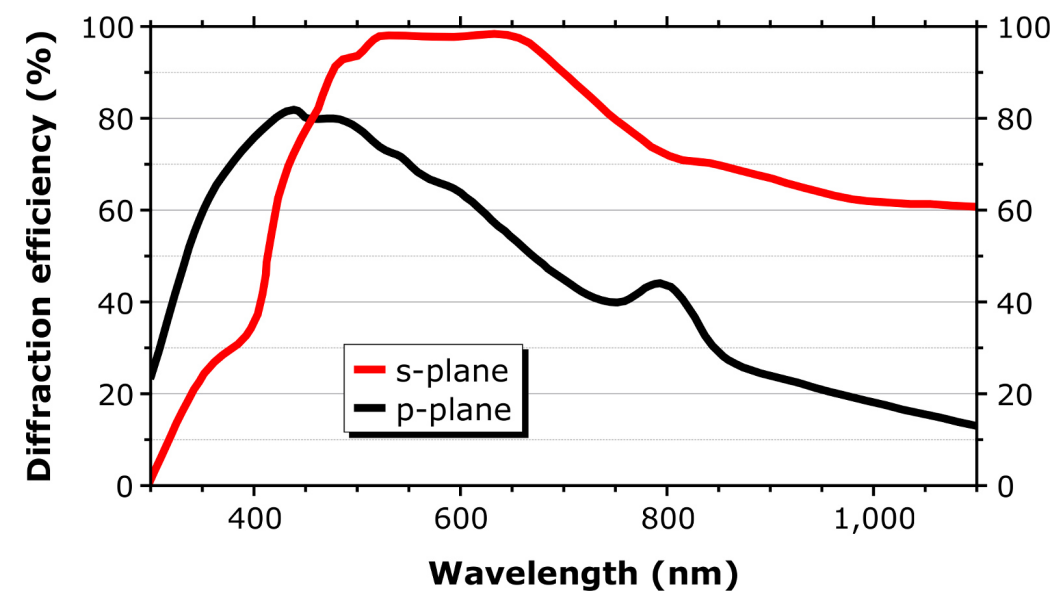

FIG. B.4: Efficiency curves for the reflection grating from Richardson Optics. The graphs are taken from the specification sheet and normalized to the reflection of aluminium. As the grating is optimized for silicon absorption measurements, it was installed in the monochromator Omni $\lambda-150$ from Gilden Photonics in an astigmatism-corrected Czerny-Turner design. The blazing wavelength is at $500 \mathrm{~nm}$, and the grating's period of $833 \mathrm{~nm}$ corresponds to a groove density of 1200 grooves $/ \mathrm{mm}$. 


\section{B.1.3 The detectors}

For measuring the intensity of the diffuse light in the sphere, a photo-detector needs to be mounted at the sphere's detector port (see Fig. B.1). Since the setup has to characterize the absorption of (structured) silicon samples, a high sensitivity is required in the NIR spectral region, where silicon is only weakly absorbing. In the following, the requirements of the detector are analyzed in terms of bandwidth, sensitivity, performance and suitability.

Detector bandwidth The scanning motor of the monochromator samples $A(\lambda)$ at intervals of $\Delta \lambda=1 \mathrm{~nm}$. The sampling rate $f_{s}$ is given by the grating motor, which is limited to a maximum speed of $7 \mathrm{~Hz}$, hence the detector bandwidth does not need to exceed $2 f_{s}=14 \mathrm{~Hz}$, according to the sampling theorem. A low bandwidth also means that the $30 \mathrm{kHz}$ pulse repetition rate of the laser is not resolved (as required) and the detector reads out a constant DC signal.

While the absorption of a sample (mounted in the center of the sphere) is derived by the signal of the detector that is directly attached to the sphere, fluctuations of the laser light, occurring during the measurements, are taken into account by a second detector outside the sphere. The arrangement of the detectors is schematically shown in Fig. B.5.

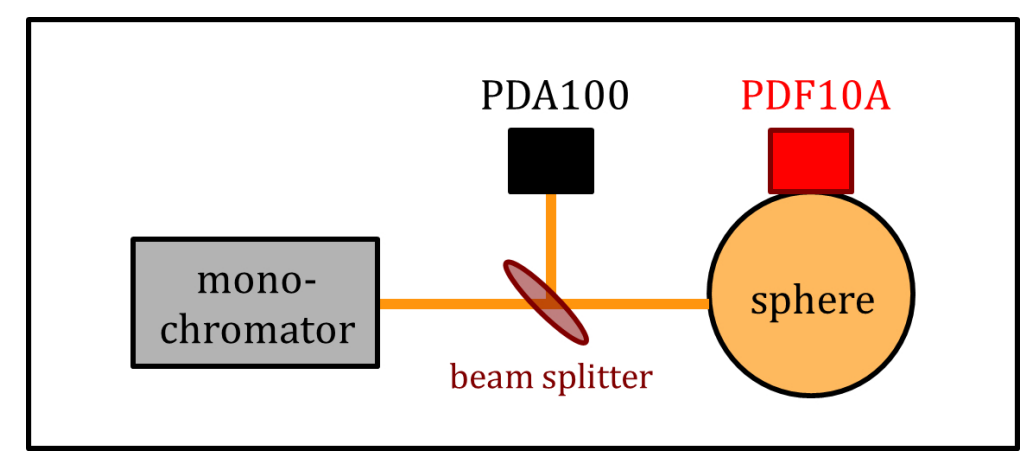

FIG. B.5: The signal detector (highlighted in red) records the radiance inside the sphere and is directly attached via a port reducer. Any fluctuations of the laser light are taken into account by a reference detector (highlighted in black) outside the sphere. Since the monochromator acts like a gate for the selected wavelength, the sampling rate is defined by the speed of the stepping motor and not by the bandwidth of the detectors. 
Sensitivity The sensitivity of a photodetector is related to the signal-to-noise power ratio $S / N$. The noise equivalent power contained in a $1 \mathrm{~Hz}$ bandwidth is quantified by

$$
N E P \equiv \frac{\text { RMS noise voltage }(V / \sqrt{1 \mathrm{~Hz}})}{\text { spectral responsivity }(\mathrm{A} / \mathrm{W}) \times \operatorname{transimpedance~gain~}(\mathrm{V} / \mathrm{A})}
$$

The spectral responsivity is typically in the order of $0.5 \mathrm{~A} / \mathrm{W}$; if the RMS noise voltage of the detector is above $2.5 \mathrm{mV} / \sqrt{\mathrm{Hz}}$, a gain of $10^{12} \mathrm{~V} / \mathrm{A}$ is required to obtain a $N E P$ of $5 \mathrm{fW} / \sqrt{\mathrm{Hz}}$.

As the bandwidth does not need to exceed $14 \mathrm{~Hz}$ (see section B.1.3), the detector is used in photovoltaic mode, i.e. without externally applied bias, because of reduced dark current and higher linearity at low light levels, which are not as good when a reverse bias is applied (see Fig. B.6).

The minimum power $P_{\min }$ required for a $S / N=1$ is then

$$
P_{\text {min }}=N E P \cdot \sqrt{\Delta f_{d}} \quad \text { with } f_{d} \leq 14 \mathrm{~Hz}
$$

For example, if the NEP of a detector is $5 \mathrm{fW} / \sqrt{\mathrm{Hz}}$ at $2 \mathrm{eV}$ (620 nm wavelength), $P_{\text {min }}=18.7 \mathrm{fW}$ in a bandwidth of $14 \mathrm{~Hz}$, i.e. more than 4200 photons are required to obtain a $S / N>1(4200 \times 14 \mathrm{~Hz} \times 2 \mathrm{eV} \approx 18.8 \mathrm{fW})$.

In order to compare various detectors and to understand their performances, the $N E P$ is normalized to the square root of the active area $A$. The resulting quantity is commonly referred to as the sensitivity, or specific detectivity $D^{*}$, of a photodetector:

$$
D^{*} \equiv \frac{\sqrt{A}}{N E P}=\frac{\sqrt{A \Delta f_{d}}}{P_{\min }} \quad \Longrightarrow \quad S / N=D^{*} \cdot \frac{P_{\text {in }}}{\sqrt{A \cdot \Delta f_{d}}}
$$

The sensitivity is measured in Jones $[\mathrm{cm} \sqrt{\mathrm{Hz}} / \mathrm{W}]$ and is the most useful parameter for the purpose of comparison, because $D^{*}$ is independent of the area or operating frequency. 


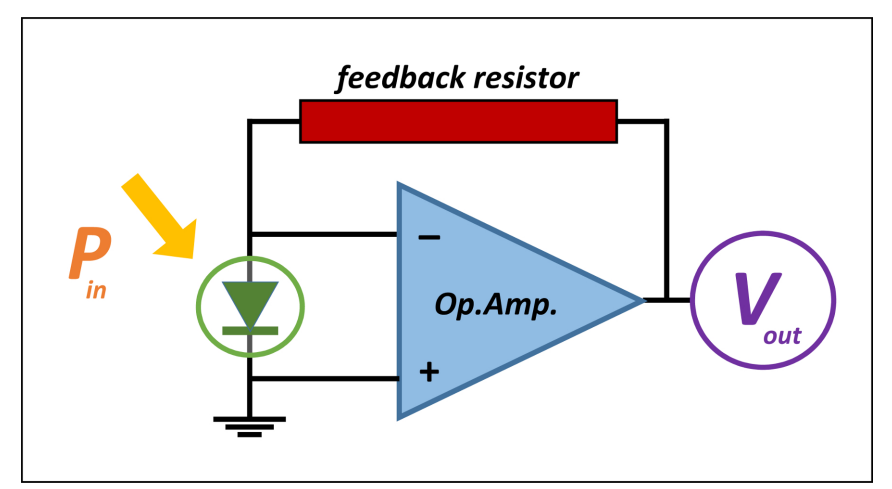

FIG. B.6: When photodetectors are operated in the photovoltaic mode, the $p n$-diode remains zero biased. As the open-circuit voltage depends on the incident light intensity $\Phi_{i n}$ logarithmically, the photodiode is virtually shorted to the ground of an operational amplifier and therefore used as a current generator instead. A large feedback resistor then serves as a (negative) feedback response to the photodiode, implying a high amplifier gain, yet a slow voltage response $V_{\text {out }}$. However, the operation is extremely linear at low light levels due to the almost complete elimination of leakage currents.

A different figure-of-merit is often cited as the gain-bandwidth product (GBP), because both a high gain and a high cut-off frequency are advantageous. However, since the noise power $P_{\min }$ scales with the operating bandwidth $\Delta f_{d}$ according to Eq. 2, transimpedance amplifiers are specifically designed for the operation at low frequencies (small bandwidth) with very large feedback resistors (high gain).

Four noise sources are mainly encountered in connection with photodetectors:

1. Johnson noise. Johnson noise results from the random motion of electrons in a conductor and is the dominant contribution to the overall noise figure if a large feedback resistor is used in the photovoltaic mode of operation. Johnson noise has a white noise spectrum (a flat frequency power spectrum).

2. Shot noise. Shot noise, or dark current noise, results from the random generation and recombination of charge carriers within the depletion region in the absence of an external signal. Shot noise provides the greatest noise contribution in the photoconductive mode of operation, because it scales with the reverse bias. 
3. Photon noise. The nature of the signal itself may lead to appreciable changes in the photocurrent: photon noise, also known as Poisson noise, refers to the inherent variation of the incident photon flux and, therefore, imposes a fundamental noise limit to a photodetector. One way to reduce this kind of noise is by increasing the signal level, which usually means longer integration times.

4. $1 / \mathrm{f}$ noise. While the mechanisms that produce $1 / \mathrm{f}$ noise are poorly understood, it is often related to the DC level and to imperfections in the semiconductor. 1/f noise is a typical noise figure of transistors and considered to be correlated with an inefficient charge transfer of the channels. In presence of $1 / \mathrm{f}$ noise, longer integration times do not improve the $S / N$ and possibly saturate the pixel. $1 /$ f noise has a pink noise spectrum, i.e. every octave contains the same amount of noise power.

In addition, if the setup is not sufficiently screened, background signals can influence the noise floor of the detector. For example, external noise sources created by electrical devices tend to occur at the power line frequency or any harmonic of it. It is therefore a common practice to avoid sampling frequencies that are equal to an integer number of power line cycles (NPLC).

Optical powers down to the sub-femtowatt regime, however, are generally only detectable in combination with a phase-sensitive device, e.g. a lock-in amplifier. The lock-in amplifier is able to sample a small AC signal while ignoring noise signals at other frequencies. Using a lock-in therefore requires to periodically interrupt a continuous signal, e.g. a light source by a chopper. The modulation allows to choose an operating frequency where any interfering signals are smallest.

I decided not to use a lock-in amplifier, because a lock-in typically operates at several $100 \mathrm{~Hz}$, which is higher than the required bandwidth of the detector. In addition, if the voltage across the diode is (virtual) zero, the dark current is minimized, thus the noise at the amplifier input remains almost constant over time, and hence the $1 / \mathrm{f}$ noise is reduced significantly in the photovoltaic mode of operation. For low DC currents, the thermal noise in the large feedback resistor will therefore provide the greatest contribution to the overall noise figure. However, 
the $S / N$ then can always be increased by a slower sampling rate $f_{s}$ and longer integration time $1 / f_{d}$. At low light levels, it is better to minimize external noise sources and to choose a detector with a small bandwidth and low NEP, than to extract the signal from the noise after its detection.

\section{B.2 Critical assessment}

In summary, the combination of detector and spectrum analyzer into a single package as in the USB4000 is not advantageous for the detection of weak and diffusive light. The separation of dispersion and detection into two specialised units increases the resolution, sensitivity and flexibility of the experiment. The setup can easily be extended for accurate measurements of quantum efficiencies.

The requirements for the detector now are:

1. bandwidth of less than $20 \mathrm{~Hz}$,

2. gain in the order of $10^{12} \mathrm{~V} / \mathrm{A}$,

3. high responsivity in the NIR spectral region $(>0.5 \mathrm{~A} / \mathrm{W})$,

4. active area adapted to the sphere's detector port $\left(<25 \mathrm{~mm}^{2}\right)$,

5. low $\operatorname{NEP}(<5 \mathrm{fW} / \sqrt{H z})$.

Silicon photo-detectors are sensitive to light from the UV to the NIR spectral region (350 $\mathrm{nm}$ to $1100 \mathrm{~nm}$ ), as already shown in Fig. B.2. Thorlabs offers various models with active areas between 1 and $75 \mathrm{~mm}^{2}$, peak responsivities around $970 \mathrm{~nm}$ wavelength and gain figures from $10^{3}$ up to $10^{12}$.

The silicon detector PDF10A by Thorlabs fulfills all the above listed requirements and was therefore directly attached to the integrating sphere. The reference detector PDA100 is used outside the sphere to monitor any intensity fluctuations of the laser beam. The specifications of the detectors are listed in Tab. B.1 and their performance compared to the USB4000 spectrometer in Tab. B.2.

By comparing the key parameters listed in Tab. B.2, the high gain and sensitivity of the PDF10A together with two Keithly digital multimeters (DMM) present the best option. Figure B.7 shows a photograph of the final measurement setup. 


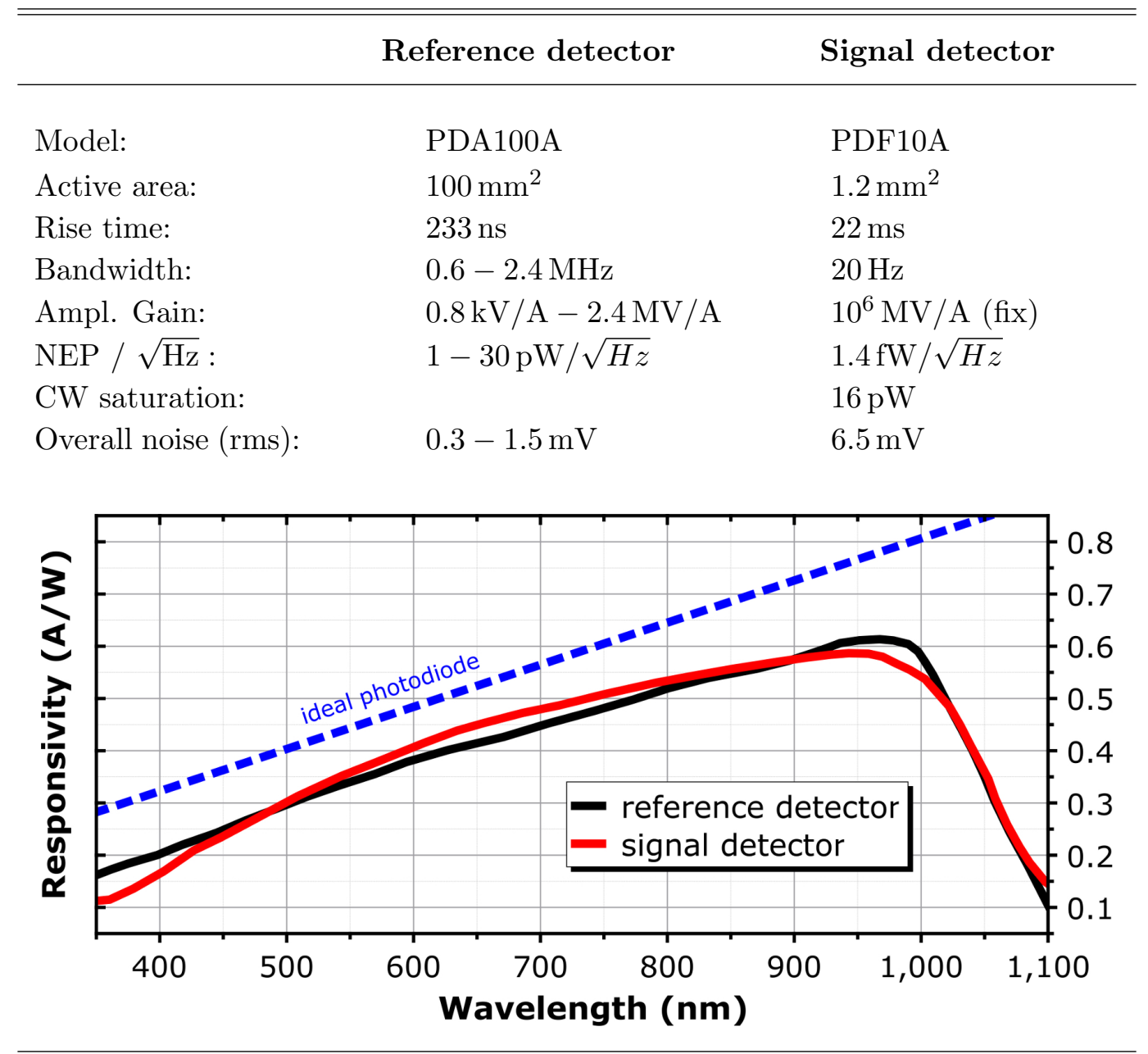

TAB. B.1: Spectral response of the two Si photodetectors that are used in the absorption measurement setup. The red solid line refers to the PDF10A detector (signal detector), which is directly attached to the sphere, whereas the black solid line refers to the PDA100 detector (reference detector), which is positioned outside the sphere, c.f. Fig. B.5. The spectral response curves are typical for silicon, with the peak position being defined by the thickness of the silicon absorber layer. The measured responsivities are compared to an ideal photodiode with a direct electrical bandgap of $1.12 \mathrm{eV}$ (blue dotted line). As the ideal photodiode has a wavelength independent (external) quantum efficiency of $100 \%$, reflection losses and recombination losses of photo-generated charge carriers are the main reason for the slightly lower responsivities of the high-quality photodetectors. 


\begin{tabular}{l|rrr}
\hline \hline Detector name & USB4000 & PDA100 & PDF10A \\
\hline & 160 & $50-100$ & 240 \\
Gain $(\mathrm{dB})$ & 130 & $6 \mathrm{k}-2.4 \mathrm{M}$ & 20 \\
Bandwidth $(\mathrm{Hz})$ & $10 \mathrm{G}$ & $600 \mathrm{M}$ & $20 \mathrm{~T}$ \\
GB-Product $(\mathrm{Hz})$ & $3.5 \mathrm{p}$ & $1-30 \mathrm{p}$ & $1.4 \mathrm{f}$ \\
NEP $(\mathrm{W} / \sqrt{\mathrm{Hz}})$ & $1.6 \cdot 10^{-4}$ & 100 & 1.2 \\
$\mathrm{~A}\left(\mathrm{~mm}{ }^{2} / \mathrm{pixel}\right)$ & $360 \mathrm{M}$ & $30 \mathrm{G}-1 \mathrm{~T}$ & $70 \mathrm{~T}$ \\
$D^{*}\left(\mathrm{Jonnes}^{2}\right.$ & &
\end{tabular}

TAB. B.2: The parameters as listed are taken or calculated from the datasheets. The PDA100A has switchable gain, allowing to adapt its sensitivity to the light conditions. The USB4000 differs from the PDx detectors by the CCD sensor used for the data acquisition: the read-out time of all data from the CCD restricts the integration time between $3.8 \mathrm{~ms}$ and $10 \mathrm{~s}$. The bandwidth can be increased up to $100 \mathrm{kHz}$ by balancing the loss in gain via signal averaging. The low detectivity is mainly due to the pixel size, such that a higher detectivity would necessarily involve a loss in optical resolution or spectral range. The high gain and sensitivity of the PDF10A make the detector suitable for the measurement of small light intensity changes in the integrating sphere (as required).

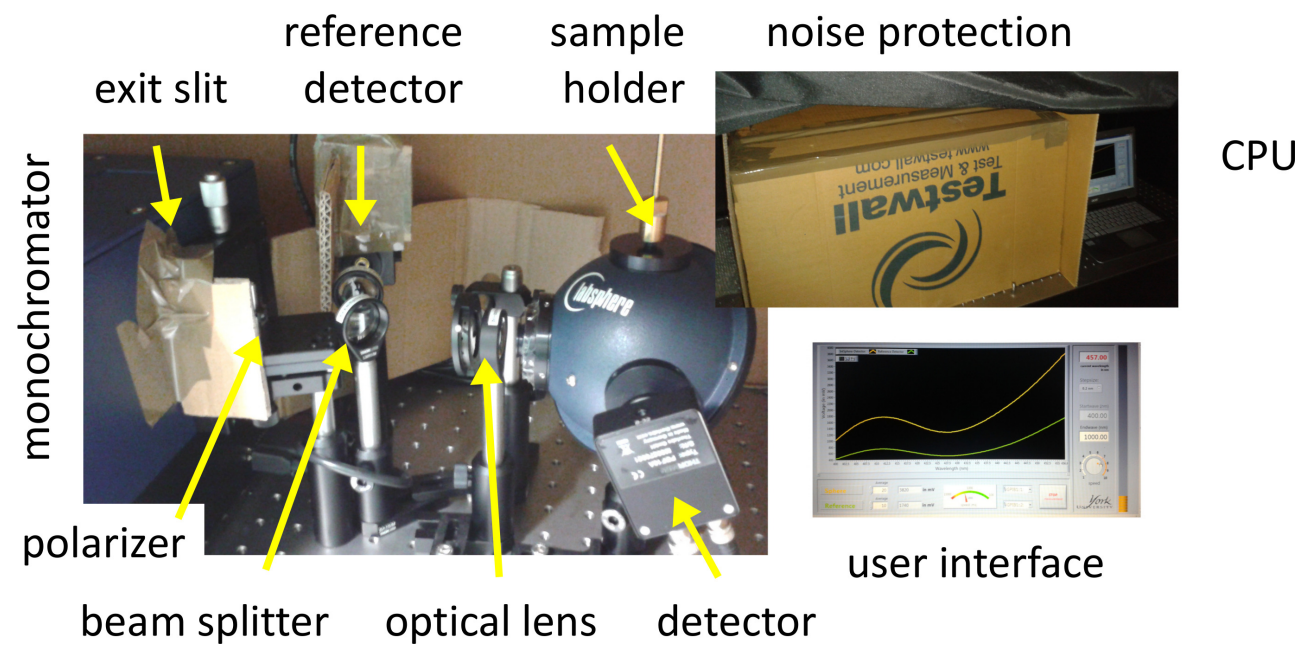

FIG. B.7: The absorption measurement setup is covered in a carton box to minimize the background noise level. The exit slit of the monochromator can be adjusted for the required optical resolution. The detector is directly attached to the integrating sphere, while a reference detector monitors the optical power of the laser beam. All data acquisitions are synchronized by a LabView program with a user interface. 


\section{B.3 Data acquisition - speed and accuracy}

Based on the settling time of the detector $(\sim 25 \mathrm{~ms})$, the Keithley digital multimeter (DMM2110) can integrate the DC signal in $2 \mathrm{~s}, 200 \mathrm{~ms}, 40 \mathrm{~ms}$ and faster time intervals. I aimed to maximize the integration period of the A/D converter at the Nyquist rate $2 f_{s}$ in order to reduce the noise from the number of power line cycles (NPLC). Once $f_{s}$ is specified, the following Tab. B.3 lists the optimal configurations along with the total acquisition times.

\begin{tabular}{l|rrrrr}
\hline \hline grating motor $f_{s}:$ & $7 \mathrm{~Hz}$ & $5 \mathrm{~Hz}$ & $2.5 \mathrm{~Hz}$ & $1 \mathrm{~Hz}$ & $0.25 \mathrm{~Hz}$ \\
detector bandwidth $f_{d}:$ & $14 \mathrm{~Hz}$ & $10 \mathrm{~Hz}$ & $5 \mathrm{~Hz}$ & $2 \mathrm{~Hz}$ & $0.5 \mathrm{~Hz}$ \\
ADC rate (NPLC): & 2 & 2 & 10 & 10 & 100 \\
total time (650 nm window): & $1.5 \mathrm{~min}$ & $2.2 \mathrm{~min}$ & $4.3 \mathrm{~min}$ & $10.8 \mathrm{~min}$ & $43.3 \mathrm{~min}$ \\
\hline
\end{tabular}

TAB. B.3: Optimal parameter settings for the measurement of $A(\lambda)$ in $1 \mathrm{~nm}$ steps. The digital multimeter allows me to measure the detector signal with a resolution of $0.1 \mathrm{mV}$ over a $10 \mathrm{~V}$ range. If the number of power line cycles (NPLC) is 10, each read-out is taken as the average of a $200 \mathrm{~ms}$ integration time with an accuracy between $\pm 0.2 \mathrm{mV}(\mathrm{S} / \mathrm{N}=1)$ and $\pm 1.4 \mathrm{mV}$ at $\mathrm{CW}$ saturation level $(\mathrm{S} / \mathrm{N}=1500)$.

\section{B.4 Measurement limitations}

Since the settling time is far less than a sampling time interval $1 / f_{s}, A(\lambda)$ can always be measured at the Nyquist rate, such that the noise of the detector is be reduced to the absolute minimum. In fact, the high sensitivity of the sensor required me to cover the setup with a carton box even in a completely dark room to avoid background noise.

The accuracy of the digital multimeter (DMM) corresponds to an intensity change of $5.2 \mathrm{fW}$ in a $14 \mathrm{~Hz}$ bandwidth. Hence, provided the incident power is above $P_{\text {min }}$, such small changes in $A(\lambda)$ should be possible to detect.

The detector voltage $V_{\text {out }}$ depends linearly on the incident power $P_{\text {in }}$ and is given by

$$
V_{\text {out }}(\lambda)=\left(10^{12} \frac{\mathrm{V}}{\mathrm{A}} \cdot \mathcal{R}(\lambda)\right) \times P_{\text {in }}(\lambda)
$$


where $\mathcal{R}$ is the wavelength dependent spectral responsivity of the detector and $10^{12} \frac{\mathrm{V}}{\mathrm{A}}$ the gain of the amplifier.

If I assume a $40 \mathrm{~dB}$ intensity reduction of the white light source due to the coupling to the monochromator, the attenuation by optical lenses, polarizer, beam splitter, iris diaphragm and diffuse integrating sphere - roughly estimating that each component leads to an intensity drop of $50 \%$-, the detector would almost saturate for a transparent sample, following Eq. 4:

$$
P_{\text {in }}^{\max }=0.5^{6} \cdot 30 \mu \mathrm{W} / A_{\text {sphere }} \approx 15 \mathrm{pW} / A_{\text {detector }} \quad \Rightarrow \quad V_{\text {out }}(960 \mathrm{~nm})=9 \mathrm{~V}
$$

$A_{\text {detector }}$ and $A_{\text {sphere }} \approx 30,000 \times A_{\text {detector }}$ represent the surface of the active area of the detector and the integrating sphere, whereas the white light source intensity $30 \mu \mathrm{W} / \mathrm{nm}$ and detector's responsivity $0.6 \mathrm{~A} / \mathrm{W}$ at $960 \mathrm{~nm}$ wavelength are taken from Fig. B.3 and Tab. B.1, respectively.

If I assume the sample not to be transparent but $99 \%$ absorbing, the previous $40 \mathrm{~dB}$ intensity reduction would drop to almost $80 \mathrm{~dB}\left(0.01 \cdot 0.5^{6} \approx 10^{-4}\right)$. However, the signal would still remain above the detection limit of $6 \mathrm{mV}$ :

$P_{\text {in }}^{\min }=10^{-4} \cdot 20 \mu \mathrm{W} / A_{\text {sphere }} \approx 70 \mathrm{fW} / A_{\text {detector }} \quad \Rightarrow \quad V_{\text {out }}(400 \mathrm{~nm})=15 \mathrm{mV}$

These assumption were indeed reasonably well verified in the measurements.

In conclusion, the PDF10A fulfils all requirements of accurate absorption measurements and allows us to characterise $A(\lambda)$ over a broad spectral range within a total acquisition time of less than 2 minutes at sub-nm optical resolution. 


\section{Bibliography}

[1] Marcus Ejder and Richard T. Carlsen. Cost drivers in the photovoltaic solar industry. KTH - School of Industrial Engineering and Management, Stockholm (SE), 2011. Bachelor thesis.

[2] A. Jäger-Waldau. Pv status report 2014. European Union, 2014. URL http://dx.doi.org/doi:10.2790/941403.

[3] Roeder-Johnson Corporation. Hanergy completes acquisition of alta devices. Press information, May 2015. URL http://www.altadevices . $\mathrm{com} / \mathrm{pr}-2014-08-13 \cdot \mathrm{php}$.

[4] Wikipedia. Nanosolar, abound solar, solyndra. Wikipedia, The Free Encyclopedia, May 2015. URL http://en.wikipedia.org/.

[5] Mark Kerstens. Solexel unveils ultra-thin, high-performance silicon solar cell at intersolar. Press release, July 2012. URL http://www.solexel. com/IntersolarNA2012.php.

[6] Jesse Noffsinger, Emmanouil Kioupakis, Chris G. Van de Walle, Steven G. Louie, and Marvin L. Cohen. Phonon-assisted optical absorption in silicon from first principles. Phys. Rev. Lett., 108(167402), 2012.

[7] Michal Lucki and Tomas Zeman. Dispersion Compensating Fibres for Fibre Optic Telecommunication Systems. InTech, 2015. doi: 10.5772/59152.

[8] Peter Würfel. Physics of Solar Cells. Wiley, 2005.

[9] S. Sandhu, Z. Yu, and S. Fan. Detailed balance analysis of nanophotonic solar cells. OpEx, 21(1):1209-1217, January 2013. 
[10] S. Sandhu, Z. Yu, and S. Fan. Detailed balance analysis and enhancement of open-circuit voltage in single-nanowire solar cells. Nano Lett., 14(2): 1011-1015, January 2014.

[11] M. A. Green and M. J. Keevers. Short communication: Optical properties of intrinsic silicon at $300 \mathrm{k}$. Prog. in PV: research and applications, 3: 189-192, 1995.

[12] MA Green. Solar cell fill factors: General graph and empirical expressions. Solid-State Electronics, 24:788-789, 1981.

[13] W. Shockley and H. J. Queisser. Detailed balance limit of efficiency of pn junction solar cells. AP, 32(3):510-519, 1961.

[14] W. Marx. The shockley-queisser paper - a notable example of a scientific sleeping beauty. Ann. Phys., 526(5-6):A41-A45, 2014.

[15] M. Gharghi, E. Fathi, B. Kante, S. Sivoththaman, and X. Zhang. Heterojunction silicon microwire solar cells. Nano Lett., 12(12):6278-6282, November 2012.

[16] Haoting Shen. Radial junction solar cells based on heterojunction with intrinsic thin layer (hit) structure. Dissertation, The Pennsylvania State University:167, 2014.

[17] J. Oh, H-C. Yuan, and H. M. Branz. An 18.2\%-efficient black-silicon solar cell achieved through control of carrier recombination in nanostructures. Nature Nanotechnology, 7:743-748, September 2012.

[18] X. X. Lin, X. Hua, Z. G. Huang, and W. Z. Shen. Realization of high performance silicon nanowire based solar cells with large size. Nanotechnology, 24(235402), May 2013.

[19] Haoting Shen. Effect of c-si doping density on heterojunction with intrinsic thin layer (hit) radial junction solar cells. 39th IEEE Photovoltaic Specialists Conference (PVSC), The Pennsylvania State University:24662469, June 2013. 
[20] H. A. Atwater and A. Polman. Plasmonics for improved photovoltaic devices. Nature Materials, 9:205-213, 2010.

[21] J. Park, J. Rao, T. Kim, and S. Varlamov. Highest efficiency plasmonic polycrystalline silicon thin-film solar cells by optimization of plasmonic nanoparticle fabrication. Plasmonics, 8(2):1209-1219, March 2013.

[22] V. E. Ferry, M. A. Verschuuren, H. B. T. Li, E. Verhagen, R. J. Walters, R. E. I. Schropp, H. A. Atwater, and A. Polman. Light trapping in ultrathin plasmonic solar cells. Opt. Express, 18(S2), 2010.

[23] Hairen Tan, Rudi Santbergen, Arno H. M. Smets, and Miro Zeman. Plasmonic light trapping in thin-film silicon solar cells with improved selfassembled silver nanoparticles. Nano Lett., 12:4070-4076, 2012.

[24] Hairen Tan, Laura Sivec, Baojie Yan, Rudi Santbergen, Miro Zeman, and Arno H. M. Smets. Improved light trapping in microcrystalline silicon solar cells by plasmonic back reflector with broad angular scattering and low parasitic absorption. Appl. Phys. Lett., 102(153902), 2013.

[25] Seweryn Morawiec, Manuel J. Mendes, Sergej A. Filonovich, Tiago Mateus, Salvatore Mirabella, Hugo Águas, Isabel Ferreira, Francesca Simone, Elvira Fortunato, Rodrigo Martins, Francesco Priolo, and Isodiana Crupi. Broadband photocurrent enhancement in a-si:h solar cells with plasmonic back reflectors. Opt. Exp., 22(S4), 2014.

[26] P. Spinelli, M.A. Verschuuren, and A. Polman. Broadband omnidirectional antireflection coating based on subwavelength surface mie resonators. $\mathrm{Na}$ ture Com., 3(692), 2012.

[27] J. Grandidier, D. M. Callahan, J. N. Munday, and H. A. Atwater. Light absorption enhancement in thin-film solar cells using whispering gallery modes in dielectric nanospheres. Adv. Mater., 23(10):1272-1276, 2011.

[28] E. Garnett and P. Yang. Light trapping in silicon nanowire solar cells. Nano Lett., 10:1082-1087, January 2010. 
[29] R. Santbergen, R. Liang, and M. Zeman. a-si:h solar cells with embedded silver nanoparticles. 35th IEEE Photovoltaic Specialists Conference (PVSC), pages 748-753, June 2010.

[30] J. Müller, B. Rech, J. Springer, and M. Vanecek. Tco and light trapping in silicon thin film solar cells. Sol. Energy, 77(6):917-930, 2004.

[31] G.F. Zheng, J. Zhao, M. Gross, and E. Chen. Very low light-reflection from the surface of incidence of a silicon solar cell. Sol. Energy Mat. and Solar Cells, 40(1):89-95, March 1996.

[32] S. Han, B. K. Paul, and C. Chang. Nanostructured ZnO as biomimetic antireflective coatings on textured silicon using a continuous solution process. J. Mater. Chem., 22(43):22906-22912, 2012.

[33] E. Jiménez-Rodríguez A. Montesdeoca-Santana, B. González-Díaz, D. Borchert, and R. Guerrero-Lemus. Ultra-low concentration $\mathrm{na}_{2} \mathrm{CO}_{3} /$ nahco $_{3}$ solution for texturization of crystalline silicon solar cells. Prog. PV, 20(2):191-196, March 2012.

[34] B. Bläsi. Examples of photonic microstructures. February 2015. URL http://www.ise.fraunhofer.de/en/fields-of-expertise/ optics-and-photonics/applications/structure-examples.

[35] A. Fresnel. Mémoires sur la diffraction de la lumière. Oeuvres, 1:89-122, 1816.

[36] Stéphane Larouche and David R. Smith. Reconciliation of generalized refraction with diffraction theory. Optics Letters, 37(12):2391-2393, 2012.

[37] Charles Kittel. Introduction to Solid State Physics. Wiley, 2004.

[38] J. D. Joannopoulos, S. G. Johnson, J. N. Winn, and R. D. Meade. Photonic crystals - molding the flow of light. Princeton University Press, 2007.

[39] E. Yablonovitch and G. D. Cody. Intensity enhancement in textured optical sheets for solar cells. IEEE Transactions on Electron Devices, 29(2):300$305,1982$. 
[40] J. H. Lambert. Photometria, sive de mensura et gradibus luminis, colorum et umbrae. W. Engelmann, Leipzig, 1892.

[41] J. Gee. The effect of parasitic absorption losses on light trapping in thin silicon solar cells. IEEE PVSC Las Vegas, 1:549-554, 1988.

[42] C. Battaglia, M. Boccard, F-J. Haug, and C. Ballif. Light trapping in solar cells: When does a lambertian scatterer scatter lambertianly? Applied Physics, 112(094504), November 2012.

[43] A. Bozzola, M. Liscidini, and L. C. Andreani. Photonic light-trapping versus lambertian limits in thin film silicon solar cells with $1 \mathrm{~d}$ and $2 \mathrm{~d}$ periodic patterns. Opt. Express, 20(S2):A224-A244, March 2012.

[44] Z. Yu, A. Raman, and S. Fan. Fundamental limit of nanophotonic light trapping in solar cells. PNAS, 107(41):17491-17496, October 2010.

[45] Ian Marius Peters. Phase space considerations for light path lengths in planar, isotropic absorbers. Opt. Exp., 22(S3):A908-A920, 2014.

[46] A. Basch, F. J. Beck, T. Söderström, S. Varlamov, and K. R. Catchpole. Combined plasmonic and dielectric rear reflectors for enhanced photocurrent in solar cells. APL, 100(243903), June 2012.

[47] F. Pratesi, M. Burresi, F. Riboli, K. Vynck, and D. S. Wiersma. Disordered photonic structures for light harvesting in solar cells. OpEx, 21(S3):A460A468, April 2013.

[48] R.S. Ohl. Light-sensitive electric device, 1946. URL http://www.google. com/patents/US2402662. US Patent 2402662.

[49] I. M. Ross. The invention of the transistor. IEEE, 86(1):7-28, January 1998.

[50] D. M. Chapin, C. S. Fuller, and G. L. Pearson. A new silicon p-n junction photocell for converting solar radiation into electrical power. AP, 25: 676/677, 1954. 
[51] G. K. Teal. Some recent developments in silicon and germanium materials and devices. National Conference on Airborne Electronics Dayton (Ohio), 1954.

[52] G. K. Teal and J. B. Little. Growth of germanium single crystals. Phys. Rev., 78:647, 1950.

[53] A. W. Blakers and M. A. Green. 20\% efficiency silicon solar cells. APL, 48 (3):215-217, 1986.

[54] C. R. Baraona and H. W. Brandhorst. V-grooved silicon solar cells. IEEE 11th Photovoltaic Specialists Conf. in Phoenix (Arizona), 1975.

[55] M. S. Bae and R. V. O'Aielio. $\mathrm{p}+/ \mathrm{n}$ high-efficiency silicon solar cells. APL, 31(285), 1977.

[56] P. Sheng, A. N. Bloch, and R. S. Stepleman. Wavelengthselective absorption enhancement in thinfilm solar cells. Appl. Phys. Lett., 43(6):579-581, September 1983.

[57] H. W. Deckman, C. B. Roxlo, and E. Yablonovitch. Maximum statistical increase of optical absorption in textured semiconductor films. Opt. Lett., 8(9):491-493, 1983.

[58] H. W. Deckman, C. R. Wronski, H. Witzke, and E. Yablonovitch. Optically enhanced amorphous silicon solar cells. APL, 42(11):968-970, 1983.

[59] P. Campbell and M. A. Green. Light trapping properties of pyramidally textured surfaces. AP, 62(243), 1987.

[60] J. Zhao, A. Wang, and M.A. Green. 24\% efficient perl structure silicon solar cells. IEEE Photovoltaic Specialists Conference (Kissimmee), 1:333$335,1990$.

[61] E. Yablonovitch. Inhibited spontaneous emission in solid-state physics and electronics. Phys. Rev. Lett., 58(20):2059-2062, 1987.

[62] J. Szlufcik, S. Sivoththaman, J. F. Nijs, R. P. Mertens, and R. van Overstraeten. Low-cost industrial technologies of crystalline silicon solar cells. IEEE, 85(5):711-730, 1997. 
[63] S. Chou, P. Krauss, and P. Renstrom. Imprint lithography with 25nanometer resolution. Science, 272(5258):85-87, 1996.

[64] A. Gombert, K. Rose, A. Heinzel, W. Horbelt, C. Zanke, B. Bläsi, and W. Wittwer. Antireflective submicrometer surface-relief gratings for solar applications. SEM, 54:333-342, 1998.

[65] K. Yamamoto, M. Yoshimi, T. Suzuki, Y. Tawada, Y. Okamoto, and A. Nakajima. Thin film poly-si solar cell on glass substrate fabricated at low temperature. MRS Spring meeting on amorphous and microcrystalline silicon technology (San Francisco), 507:131-138, 1998.

[66] J. Zhao, A. Wang, M. A. Green, and F. Ferrazza. 19.8\% efficient "honeycomb" textured multicrystalline and $24.4 \%$ monocrystalline silicon solar cells. APL, 73(14):1991-1993, 1998.

[67] M. A. Green, K. Emery, Y Hishikawa, W. Warta, and E D. Dunlop. Solar cell efficiency tables (version 43). Prog. PV: Res. Appl., 22:1-9, 2014.

[68] R. Sinton, Y. Kwark, J. Gan, and R. Swanson. 27.5-percent silicon concentrator solar cells. IEEE Electron Device Letters, 7(10):567-569, October 1986.

[69] J. Hylton. Light coupling and light trapping in alkaline etched multicrystalline silicon wafers for solar cells. PhD thesis, University of Utrecht, 2006 .

[70] R. Einhaus, E. Vazsonyi, J. Szlufcik, J. Nijs, and R. Mertens. Isotropic texturing of multicrystalline silicon wafers with acidic texturing solutions. IEEE Photovoltaic Specialists Conference Anaheim (CA), pages 167-170, 1997.

[71] M. G. Moharam, E. B. Grann, and D. A. Pommet. Formulation for stable and efficient implementation of the rigorous coupled-wave analysis of binary gratings. J. OSA, 12(5):1068-1076, 1995.

[72] D. M. Whittaker and I. S. Culshaw. Scattering-matrix treatment of patterned multilayer photonic structures. PRB, 60(2610), 1999. 
[73] A. Shah, P. Torres, R. Tscharner, N. Wyrsch, and H. Keppner. Photovoltaic technology: The case for thin-film solar cells. Science, 285(5428):692-698, 1999.

[74] C. Battaglia, C-M. Hsu, K. Söderström, J. Escarré, F-J. Haug, M. Charrière, M. Boccard, M. Despeisse, D. T. L. Alexander, M. Cantoni, Y. Cui, and C. Ballif. Light trapping in solar cells: Can periodic beat random? ACS Nano, 6(3):2790-2792, 2012.

[75] O. Isabella, A. Ingenito, D. Linssen, and M. Zeman. Front/rear decoupled texturing in refractive and diffractive regimes for ultra-thin silicon-based solar cells. Renewable Energy and the Environment, OSA Technical Digest, paper PM4C.2, 2013.

[76] K. X. Wang, Z. Yu, V. Liu, Y. Cui, and S. Fan. Absorption enhancement in ultrathin crystalline silicon solar cells with antireflection and light-trapping nanocone gratings. Nano Lett., 12(3):1616-1619, 2012.

[77] Christian S. Schuster, Piotr Kowalczewski, Emiliano R. Martins, Maddalena Patrini, Mark G. Scullion, Marco Liscidini, Liam Lewis, Christopher Reardon, Lucio C. Andreani, and Thomas F. Krauss. Dual gratings for enhanced light trapping in thin-film solar cells by a layer-transfer technique. Opt. Expr., 21(S3):A433-A439, May 2013.

[78] J. Gjessing. Photonic crystals for light trapping in solar cells. PhD thesis, University of Oslo, 2011.

[79] D. Macdonald, A. Cuevas, M. Kerr, C. Samundsett, D. Ruby, S. Winderbaum, and A. Leo. Texturing industrial multicrystalline silicon solar cells. Solar Energy, 76:277-283, 2004.

[80] O. Isabella, K. Jäger, J. Krc, and M. Zeman. Light scattering properties of surfacetextured substrates for thin-film solar cells. Proc. 23rd EUPVSEC, 1:476-481, 2008.

[81] A. Čampa, J. Krč, F. Smole, and M. Topič. Potential of diffraction gratings for implementation as a metal back reflector in thin-film silicon solar cells. TSF, 516(20):6963-6967, 2008. 
[82] H. Sai, H. Fujiwara, and M. Kondo. Back surface reflectors with periodic textures fabricated by self-ordering process for light trapping in thin-film microcrystalline silicon solar cells. SEM, 93:1087-1090, 2009.

[83] A. Ingenito, O. Isabella, and M. Zeman. Experimental demonstration of $4 n 2$ classical absorption limit in nanotextured ultrathin solar cells with dielectric omnidirectional back reflector. ACS Photonics, 1(3):270-278, February 2014.

[84] L. Zeng, Y. Yi, C. Hong, J. Liu, N. Feng, X. Duan, LC. Kimerling, and BA. Alamariu. Efficiency enhancement in si solar cells by textured photonic crystal back reflector. APL, 89(11), 2006.

[85] P. Bermel, C. Luo, L. Zeng, LC. Kimerling, and JD. Joannopoulos. Improving thin-film crystalline silicon solar cell efficiencies with photonic crystals. OE, 15(25):16986-17000, 2007.

[86] A. Poruba, A. Fejfar, Z. Remes, J. Springer, M. Vanecek, J. Kocka, J. Meier, P. Torres, and A. Shah. Optical absorption and light scattering in microcrystalline silicon thin films and solar cells. AP, 88(1):148-160, 2000 .

[87] G. D. Cody, T. Tiedje, B. Abeles, B. Brooks, and Y. Goldstein. Disorder and the optical-absorption edge of hydrogenated amorphous silicon. Phys. Rev. Let., 47(20):1480, 1981.

[88] N. Bakr, A. Funde, V. Waman, M. Kamble, R. Hawaldar, D. Amalnerkar, S. Gosav, and S. Jadkar. Determination of the optical parameters of asi:h thin films deposited by hot wire-chemical vapour deposition technique using transmission spectrum only. Pramana, 76(3):519-531, March 2011.

[89] Am1.5g solar spectrum irradiance data, 2015. URL http://rredc.nrel. gov/solar/spectra/am1.5.

[90] E.D. Palik. Handbook of optical constants of solids. Academic, Orlando, 1985. 
[91] J. Gjessing, A. S. Sudbø, and E. S. Marstein. Comparison of periodic lighttrapping structures in thin crystalline silicon solar cells. J. Appl. Phys., 110(3):033104, 2011.

[92] E. R. Martins, J. Li, Y. Liu, V. Depauw, Z. Chen, J. Zhou, and T. F. Krauss. Deterministic quasi-random nanostructures for photon control. Nat. Commun., 4(2665), 2013.

[93] S. E. Han and G. Chen. Toward the lambertian limit of light trapping in thin nanostructured silicon solar cells. Nano Lett., 10(11):4692-4696, 2010.

[94] S. B. Mallick, M. Agrawal, and P. Peumans. Optimal light trapping in ultra-thin photonic crystal crystalline silicon solar cells. Opt. Express, 18 (6):5691-5706, 2010.

[95] A. Mellor, I. Tobias, A. Marti, and A. Luque. A numerical study of biperiodic binary diffraction gratings for solar cell applications. Sol. Energy Mater. Sol. Cells, 95(12):3527-3535, 2011.

[96] A. Mellor, H. Hauser, C. Wellens, J. Benick, J. Eisenlohr, M. Peters, A. Guttowski, I. Tobías, A. Martí, A. Luque, and B. Bläsi. Nanoimprinted diffraction gratings for crystalline silicon solar cells: implementation, characterization and simulation. Opt. Express, 21(S2):A295-A304, 2013.

[97] R. Dewan and D. Knipp. Light trapping in thin-film silicon solar cells with integrated diffraction grating. J. Appl. Phys., 106(7):074901, 2009.

[98] Y. Yao, J. Yao, V. Kris Narasimhan, Z. Ruan, C. Xie, S. Fan, and Y. Cui. Broadband light management using low-q whispering gallery modes in spherical nanoshells. Nat. Commun., 3(664), 2012.

[99] N. T. Fofang, T. S. Luk, M. Okandan, G. N. Nielson, and I. Brener. Substrate-modified scattering properties of silicon nanostructures for solar energy applications. Opt. Express, 21(4):4774-4782, 2013.

[100] D. Lockau, T. Sontheimer, C. Becker, E. Rudigier-Voigt, F. Schmidt, and B. Rech. Nanophotonic light trapping in 3-dimensional thin-film silicon architectures. Opt. Express, 21(S1):A42-A52, 2013. 
[101] X. Sheng, L. Z. Broderick, and L. C. Kimerling. Photonic crystal structures for light trapping in thin-film si solar cells: Modeling, process and optimizations. Optics Commun., 314:41-47, September 2014.

[102] C. Trompoukis, O. El Daif, V. Depauw, I. Gordon, and J. Poortmans. Photonic assisted light trapping integrated in ultrathin crystalline silicon solar cells by nanoimprint lithography. Appl. Phys. Lett., 101(10):103901, 2012.

[103] V. Depauw, X. Meng, O. El Daif, G. Gomard, L. Lalouat, E. Drouard, C. Trompoukis, A. Fave, C. Seassal, and I. Gordon. Micrometer-thin crystalline-silicon solar cells integrating numerically optimized 2-d photonic crystals. IEEE J. of Photov., 4(1):215-223, 2013.

[104] J. Zhao, A. Wang, P. P. Altermatt, S. R. Wenham, and M. A. Green. $24 \%$ efficient perl silicon solar cell: Recent improvements in high efficiency silicon cell research. Sol. Energy Mater. Sol. Cells, 41(42):87-99, 1996.

[105] F. Feldmann, M. Bivour, C. Reichel, M. Hermle, and S.W. Glunz. A passivated rear contact for high-efficiency n-type si solar cells enabling high voc's and $f f>82 \%$. 28th EU PVSEC, 2CO.4.4, 2013.

[106] L. Wang, J. Han, A. Lochtefeld, A. Gerger, M. Carroll, D. Stryker, S. Bengtson, M. Curtin, H. Li, Y. Yao, D. Lin, J. Ji, A.J. Lennon, R.L. Opila, and A. Barnett. 16.8\% efficient ultra-thin silicon solar cells on steel. 28th EU PVSEC, 3DV.1.12, 2013.

[107] J. H. Petermann, D. Zielke, J. Schmidt, F. Haase, E. G. Rojas, and R. Brendel. $19 \%$ efficient and $43 \mu \mathrm{m}$ thick crystalline si solar cell from layer transfer using porous silicon. Prog. Photovolt. Res. Appl., 20(1):1-5, 2012.

[108] Hitoshi Sai, Kimihiko Saito, Nana Hozuki, and Michio Kondo. Relationship between the cell thickness and the optimum period of textured back reflectors in thin-film microcrystalline silicon solar cells. Appl. Phys. Lett., 102(053509), 2013.

[109] C. Haase and H. Stiebig. Optical properties of thin-film silicon solar cells with grating couplers. Prog. Photovolt. Res. Appl., 14(7):629-641, 2006. 
[110] C. Heine and R. Morf. Submicrometer gratings for solar energy applications. Appl. Optics, 34(14):2476-2482, May 1995.

[111] A. Lin and J. Phillips. Optimization of random diffraction gratings in thin-film solar cells using genetic algorithms. SEM, 92:1689-1696, 2008.

[112] M. Peters, C. Battaglia, K. Forberich, B. Bläsi, N. Sahraei, and A.G. Aberle. Comparison between periodic and stochastic parabolic light trapping structures for thin-film microcrystalline silicon solar cells. Opt. Exp., 20(28):29488-29499, 2012.

[113] Emiliano R. Martins, Juntao Li, YiKun Liu, Jianying Zhou, and Thomas F. Krauss. Engineering gratings for light trapping in photovoltaics: The supercell concept. Phys. Rev. B, 86(041404(R)), July 2012.

[114] Vivian E. Ferry, Marc A. Verschuuren, M. Claire van Lare, Ruud E. I. Schropp, Harry A. Atwater, and Albert Polman. Optimized spatial correlations for broadband light trapping nanopatterns in high efficiency ultrathin film a-si:h solar cells. Nano Lett., 11:4239-4245, 2011.

[115] R. Dewan, M. Marinkovic, R. Noriega, S. Phadke, A. Salleo, and D. Knipp. Light trapping in thin-film silicon solar cells with submicron surface texture. Opt. Express, 17(25):23058-23065, 2009.

[116] D. Madzharov, R. Dewan, and D. Knipp. Influence of front and back grating on light trapping in microcrystalline thin-film silicon solar cells. Opt. Express, 19(S2):A95-A107, 2011.

[117] M. A. Tsai, H. W. Han, Y. L. Tsai, P. C. Tseng, P. Yu, H. C. Kuo, C. H. Shen, J. M. Shieh, and S. H. Lin. Embedded biomimetic nanostructures for enhanced optical absorption in thin-film solar cells. Opt. Express, 19 (S4):A757-A762, 2011.

[118] A. Abass, K. Q. Le, A. Alù, M. Burgelman, and B. Maes. Dual-interface gratings for broadband absorption enhancement in thin-film solar cells. Phys. Rev. B, 85(11):115449, 2012. 
[119] X. Meng, E. Drouard, G. Gomard, R. Peretti, A. Fave, and C. Seassal. Combined front and back diffraction gratings for broad band light trapping in thin film solar cell. Opt. Express, 20(S5):A560-A571, 2012.

[120] H. B. T. Li, R.H.-J. Franken, R.L. Stolk, J.K. Rath, and R.E.I. Schropp. Mechanism of shunting of nanocrystalline silicon solar cells deposited on rough ag/zno substrates. Solid State Phenomena, 131-133:27-32, 2007.

[121] X.-M. Yana, S. Kwon, A. M. Contreras, J. Bokor, and G. A. Somorjai. Fabrication of large number density platinum nanowire arrays by size reduction lithography and nanoimprint lithography. Nanoletters, 5(4):745-748, February 2005.

[122] Jin-Seung Sohn, Duhyun Lee, Eunhyoung Cho, Hae-Sung Kim, ByungKyu Lee, Myung-Bok Lee, and Su-Jeong Suh. The fabrication of CoPt electro-deposited bit patterned media with nanoimprint lithography. Nanotechnology, 20(025302), December 2009.

[123] Helmut Schift, Sina Saxer, Sunggook Park, Celestino Padeste, Uwe Pieles, and Jens Gobrecht. Controlled co-evaporation of silanes for nanoimprint stamps. Nanotechnology, 16(5), February 2005.

[124] Valérie Geiser. Low-Stress UV-Curable Hyperbranched Polymer Nanocomposites for High-Precision Devices. $\mathrm{PhD}$ thesis, École Polytechnique Fédérale de Lausanne (EPFL), 2010.

[125] C. Peroz, S. Dhuey, M. Cornet, M. Vogler, D. Olynick, and S. Cabrini. Single digit nanofabrication by step-and-repeat nanoimprint lithography. Nanotechnology, 23(015305):5, 2012.

[126] Henri Jansen, Han Gardeniers, Meint de Boer, Miko Elwenspoek, and Jan Fluitman. A survey on the reactive ion etching of silicon in microtechnology. J. Micromech. Microeng., 6:14-28, December 1995.

[127] Aline Herman, Christos Trompoukis, Valerie Depauw, Ounsi El Daif, and Olivier Deparis. Influence of the pattern shape on the efficiency of front-side periodically patterned ultrathin crystalline silicon solar cells. J. Applied Physics, 112(113107), December 2012. 
[128] L. Zeng, P. Bermel, Y. Yi, B. A. Alamariu, K. A. Broderick, J. Liu, C. Hong, X. Duan, J. Joannopoulos, and L. C. Kimerling. Demonstration of enhanced absorption in thin film si solar cells with textured photonic crystal back reflector. APL, 93(221105), 2008.

[129] Jose L. Cruz-Campaa, Murat Okandana, Paul J. Resnicka, Peggy Clewsa, Tammy Pluyma, Robert K. Grubbsa, Vipin P. Guptaa, David Zubiab, and Gregory N. Nielsona. Microsystems enabled photovoltaics: 14.9\% efficient $14 \mu \mathrm{m}$ thick crystalline silicon solar cell. SEM, 95(2):551-558, 2011.

[130] Jan Hendrik Petermann, Dimitri Zielke, Jan Schmidt, Felix Haase, Enrique Garralaga Rojas, and Rolf Brendel. 19\%-efficient and $43 \mu \mathrm{m}$-thick crystalline si solar cell from layer transfer using porous silicon. Prog. PV, 20(1):1-5, 2012.

[131] K.R. Catchpole and A. Polman. Plasmonic solar cells. Opt. Express, 16 (26):21793-21800, 2008.

[132] M. J. Mendes, S. Morawiec, F. Simone, F. Priolo, and I. Crupi. Colloidal plasmonic back reflectors for light trapping in solar cells. Nanoscale, 7: 4796-4805, 2014.

[133] Christian S Schuster, Angelo Bozzola, Lucio C Andreani, and Thomas F Krauss. How to assess light trapping structures versus a lambertian scatterer for solar cells ? Opt. Exp., 22(S2), 2014.

[134] S. Morawiec, M. J. Mendes, S. Mirabella, F. Simone, F. Priolo, and I. Crupi. Self-assembled silver nanoparticles for plasmon-enhanced solar cell back reflectors: correlation between structural and optical properties. Nanotechnology, 24(265601), 2013.

[135] P. R. West, S. Ishii, G. V. Naik, N. K. Emani, V. M. Shalaev, and A. Boltasseva. Searching for better plasmonic materials. Laser $\&$ Photon. Rev., 4: 795-808, 2010.

[136] Z. Ouyang, S. Pillai, F. Beck, O. Kunz, S. Varlamov, K. R. Catchpole, P. Campbell, and M. A. Green. Effective light trapping in polycrystalline 
silicon thin-film solar cells by means of rear localized surface plasmons. APL, 96(261109), 2010.

[137] A. M. Pennanen and J. J. Toppari. Direct optical measurement of light coupling into planar waveguide by plasmonic nanoparticles. Opt. Exp., 21: A23-35, 2013.

[138] N. A. Bakr, A. M. Funde, V. S. Waman, M. M. Kamble, R. R. Hawaldar, D. P. Amalnerkar, S. W. Gosavi, and S. R. Jadkar. Determination of the optical parameters of a-si:h thin films deposited by hot wire-chemical vapour deposition technique using transmission spectrum only. Pramana: Journal of Physics, 76(3):519-531, March 2011.

[139] M. J. Mendes, E. Hernandez, E. Lopez, P. Garcia-Linares, I. Ramiro, I. Artacho, E. Antolin, I. Tobias, A. Marti, and A. Luque. Self-organized colloidal quantum dots and metal nanoparticles for plasmon-enhanced intermediate-band solar cells. Nanotech., 24(345402), 2013.

[140] M. Schmid, R. Klenk, M. Ch. Lux-Steiner, M. Topic, and J. Krc. Modeling plasmonic scattering combined with thin-film optics. Nanotech., 22 (025204), 2011.

[141] V. Jovanov, U. Planchoke, P. Magnus, H. Stiebig, and D. Knipp. Influence of back contact morphology on light trapping and plasmonic effects in microcrystalline silicon single junction and micromorph tandem solar cells. Sol. Energy Mater. Sol. Cells, 110:49-57, 2013.

[142] B. Liu and E. J. Heller. Multiple scattering and plasmon resonance in the intermediate regime. arXiv preprint arXiv:1403.4310, 2014.

[143] R. Brendel. Thin-film crystalline silicon solar cells: Physics and technology. Wiley-VCH, page 306, January 2003.

[144] F. J. Beck, S. Mokkapati, and K. R. Catchpole. Light trapping with plasmonic particles: beyond the dipole model. Opt. Exp., 19:25230-25241, 2011. 
[145] S. Mokkapati and K. R. Catchpole. Nanophotonic light trapping in solar cells. AP, 112(101101), 2012.

[146] G. Sun and J. B. Khurgin. Plasmonics and Plasmonic Metamaterials. World Scientific, edited by G. Shvets and I. Tsukerman 2012.

[147] C. Pahud, O. Isabella, A. Naqavi, F.-J. Haug, M. Zeman, H. P. Herzig, and C. Ballif. Plasmonic silicon solar cells: impact of material quality and geometry. Opt. Exp., 21:A786-797, 2013.

[148] U. W. Paetzold, E. Moulin, D. Michaelis, W. Boettler, C. Waechter, V. Hagemann, M. Meier, R. Carius, and U. Rau. Plasmonic reflection grating back contacts for microcrystalline silicon solar cells. APL, 99(181105), 2011.

[149] A. Ingenito, O. Isabella, and M. Zeman. Opto-electronic evaluation of thin double-textured crystalline silicon wafers. 39th IEEE Photovoltaic Specialist Conference, 2013.

[150] S. K. Ghosh and T. Pal. Interparticle coupling effect on the surface plasmon resonance of gold nanoparticles: From theory to applications. Chem. Rev., 107:4797-4862, 2007.

[151] S. B. Mallick, M. Agrawal, A. Wangperawong, E. S. Barnard, K. K. Singh, R. J. Visser, M. L. Brongersma, and P. Peumans. Ultrathin crystallinesilicon solar cells with embedded photonic crystals. Appl. Phys. Lett., 100 (053113), 2012.

[152] Erik Janssen. Ultra-thin single-crystalline silicon membrance solar cells as a light-trapping test platform, 2012. Master thesis.

[153] J. Besser, M. Baum, C. Vetter, C. Thanner, S. Sanchez-Ordonez, M. Wiemer, and T. Gessner. Thermal and uv nanoimprint lithography for applications from the micro to the nano scale. 6th Int. Conference 8 Exhibition on Integration Issues of Miniaturized Systems, MEMS, NEMS, ICs and Electronic Components, Zurich, March 2012. 
[154] Teri W. Odom, J. Christopher Love, Daniel B. Wolfe, Kateri E. Paul, and George M. Whitesides. Improved pattern transfer in soft lithography using composite stamps. Langmuir, 18:5314-5320, April 2002.

[155] A. Cattoni, E. Cambril, D. Decanini, G. Faini, and A.M. Haghiri-Gosnet. Soft uv-nil at $20 \mathrm{~nm}$ scale using flexible bi-layer stamp casted on hsq master mold. Microel. Eng., 87:1015-1018, 2010.

[156] F. Hamouda, H. Sahaf, S. Held, G. Barbillon, P. Gogol, E. Moyen, A. Aassime, J. Moreau, M. Canva, J. Lourtioz, M. Hanbücken, and B. Bartenlian. Large area nanopatterning by combined anodic aluminum oxide and soft uv-nil technologies for applications in biology. Microel. Eng., 88:2444-2446, 2011 .

[157] Antonio Parretta and Gabriele Calabrese. About the definition of "multiplier" of an integrating sphere. Int. J. Opt. \& Appl., 3(6):119-124, 2013.

[158] SL Storm, A Springsteen, and TM Ricker. The use of center mount sample holders in reflectance spectroscopy. Application Note No. 02, January 1998. URL http://www.labsphere.com/uploads/technical-guides/ The $\% 20$ Use $\% 20$ of $\% 20$ Center $\% 20$ Mount $\% 20$ Sample $\% 20$ Holders.pdf. 